\title{
Compactness and Finiteness Results for Gromov-Hyperbolic Spaces
}

(After joint works by G. Besson, G. Courtois, S. Gallot and A. Sambusetti)

\author{
G. Besson and G. Courtois
}

May 9, 2021

This is a series of lectures on Bishop-Gromov's type inequalities adapted to metric spaces. We consider the case of Gromov-hyperbolic spaces and draw consequences of these inequalities such as compactness and finiteness Theorems. This course is intended to be elementary in the sense that the necessary background is described in detail.

AMS codes: 51K10, 53C23, 53C21,53E20, 57K30

\section{Contents}

$\begin{array}{llr}0 & \text { Introduction } & 2\end{array}$

1 Lecture 123

1.1 Some assumptions . . . . . . . . . . . . . . . . 4

1.2 Geodesics . . . . . . . . . . . . . . . . . . . 4

1.3 Gromov-hyperbolic spaces . . . . . . . . . . . . . . 5

1.4 Measures ............................. 7

1.5 Groups acting by isometries . . . . . . . . . . . . 8

1.6 Entropy of $(X, d, \mu) \ldots \ldots \ldots \ldots \ldots \ldots$

1.7 Bishop-Gromov's Inequality . . . . . . . . . . . . . . . . . . 9

1.8 Main Theorem . . . . . . . . . . . . . . . . . . . 9

2 Lecture $2 \quad 10$

2.1 Quadrangle inequality for $\delta$-hyperbolic metric spaces . . . . . . . . . . . 10

2.2 A simple version of Theorem $1.8 \ldots \ldots \ldots \ldots$. . . . . . . . . . . . . . . . . . . . . . . 11

2.2 .1 Easy lemma . . . . . . . . . . . . . . . . . . . 11

2.2.2 More technical lemmas . . . . . . . . . . . . . . . . . 11

2.2 .3 Proof of Theorem $2.2 \ldots \ldots \ldots \ldots$ 
3 Lecture $3 \quad 15$

3.1 Fundamental group . . . . . . . . . . . . . . . . . . . . 15

3.2 First homology group . . . . . . . . . . . . . . . . . 16

3.3 Bounding the first Betti number . . . . . . . . . . . . 17

4 Lecture $4 \quad 21$

4.1 Busemann Spaces . . . . . . . . . . . . . . . . . . . . . . . . 21

4.2 Packings and Coverings . . . . . . . . . . . . . . . . 23

4.3 A Contraction in Busemann Spaces . . . . . . . . . . . . . . . . . 24

5 Lecture $5 \quad 26$

5.1 Growth of groups . . . . . . . . . . . . . . . . 26

5.2 A Margulis Lemma . . . . . . . . . . . . . . . . . . 28

5.3 The thin-thick decomposition . . . . . . . . . . . . . . 30

6 Lecture $6 \quad 32$

6.1 Systoles . . . . . . . . . . . . . . . . . . . 32

6.2 Bounding from below the Systole . . . . . . . . . . . . . . . 33

7 Lecture $7 \quad 35$

7.1 Marked groups . . . . . . . . . . . . . . . . 36

7.2 Finitely presented groups . . . . . . . . . . . . . . . . 39

7.3 Finiteness Theorem . . . . . . . . . . . . . . . . . . . 42

8 Lecture $8 \quad 43$

8.1 CAT(0)-spaces . . . . . . . . . . . . . . . 43

8.2 Towards a compactness result . . . . . . . . . . . . . . 45

8.2.1 Gromov-Hausdorff distance between metric spaces . . . . . . . . . 45

9 Lecture $9 \quad 47$

9.1 Compactness . . . . . . . . . . . . . . . . . . 47

9.2 Topological finiteness . . . . . . . . . . . . . . . . . . . . . . 49

9.3 Growth and entropy of groups . . . . . . . . . . . . . . . . . 50

10 Lecture 10

10.1 Algebraic entropy of groups acting on hyperbolic metric spaces . . . . . 52

10.2 Entropy of a $\delta$-hyperbolic space with a group action $\ldots \ldots \ldots 6$

\section{Introduction}

This text is the transcript of a series of lectures given by the authors during a CIMPA school (see here). It was held in Varanasi, India from December 5 till December 15, 2019; it was organised by Bankteshwar Tiwari and Athanase Papadopoulos. 
The theme is the Geometry of (some) compact Metric Spaces. Why should we care about metric spaces? In dimension $n>24$, the only known examples of manifolds with a Riemannian metric of positive sectional curvature also do carry a symmetric metric. Grosso modo these closed positively curved symmetric Riemannian manifolds are constructed using Lie groups and somehow, in large dimensions, they are the only examples we know. Concerning negative sectional curvature on closed manifolds of dimension greater than 2 the situation is a bit better since there are several series of examples, and some sporadic ones, of such manifolds not carrying symmetric metrics. Altogether, they constitute a sparse and statistically not significant sample.

On the other hand if one considers metric spaces, we get an incredible flexibility for constructing examples and we are left with an impressive zoology. Following Felix Klein's program we get fascinating properties of the groups acting on these spaces. However, the difficulty is now to give a meaning to positive or negative curvature. Great advances have recently been made in this direction. Several good notions of synthetic curvature have been introduced. Among them A. D. Aleksandrov's approach for a synthetic version of upper or lower bounds on sectional curvature for metric spaces has been fundamental; for details and references the reader is referred to the survey [ABN86] and the book [BBI01]. Recently synthetic versions of the Ricci curvature has been introduced by various authors and the reader could check the seminal article [BE85] and the book [Vil09] for a detailed introduction and lots of references. In these lectures we explore the negative curvature side and the key notion, which was introduced by M. Gromov, and which is now called Gromov-hyperbolicity (see [Gro87, GdlH90]).

These lectures intend to be a guide for reading the articles [BCGS20b, BCGS20a], the joint works mentioned in the subtitle. In the two first lectures we give the basic definitions and we progress towards compactness and finiteness results (see Theorems 3.6, 6.4, $7.18,9.3,10.8)$. One key step is the proof of a version of the celebrated Bishop-Gromov inequality (see Theorems 1.8, 2.2, 4.3, 4.10). The original Bishop-Gromov inequality has had a tremendous influence on the Riemannian Geometry of manifolds and we do hope that our version will have some impact.

We are happy to thank the organisers of this school: Athanase Papadopoulos and particularly Bankteshwar Tiwari and all the local organisers who made our stay a really exceptional experience.

\section{Lecture 1}

This series of lectures is based on the preprints [BCGS20b, BCGS20a] which contain all the details of the statements that follow. The main character is a metric space that we will usually denote by $(X, d)$. For the sake of simplicity we will always assume that the distance is symmetric that is,

$$
\forall x, y \in X, \quad d(x, y)=d(y, x) .
$$


This includes Riemannian and Finsler manifolds endowed with the distance coming from the Riemannian or symmetric Finsler metric.

Eventually we wish to study various families, denoted by $\mathcal{M}$, of metric spaces satisfying extra assumptions, endowed with the (pointed) Gromov-Hausdorff distance between metric spaces, denoted by $d_{G H}$. We then aim at proving that the metric space

$$
\left(\mathcal{M}, d_{G H}\right)
$$

is compact, where $\mathcal{M}=\{(X, d)$; metric space with extra assumptions $\}$. It is the core of this course to make precise and minimal these assumptions. The reader is supposed to be familiar with the Gromov-Hausdorff distance between metric spaces and, if necessary, is referred to [Gro07] Definition 3.4 page 72 or [BH99] page 70.

\subsection{Some assumptions}

A metric space $(X, d)$ is endowed with the topology induced by the distance function $d$. We always assume that $(X, d)$ is proper, i.e. that closed metric balls are compact. Notice that this excludes infinite dimensional Banach spaces.

\subsection{Geodesics}

A continuous curve,

$$
c:[0,1] \longrightarrow X, \quad c(0)=x \text { and } c(1)=y,
$$

is said to be rectifiable if

$$
\sup \left\{\sum_{i=0}^{k-1} d\left(x_{i}, x_{i+1}\right) ; 0=x_{0} \leq x_{1} \leq \cdots \leq x_{k}=1 \text {, subdivision of }[0,1]\right\}<+\infty \text {. }
$$

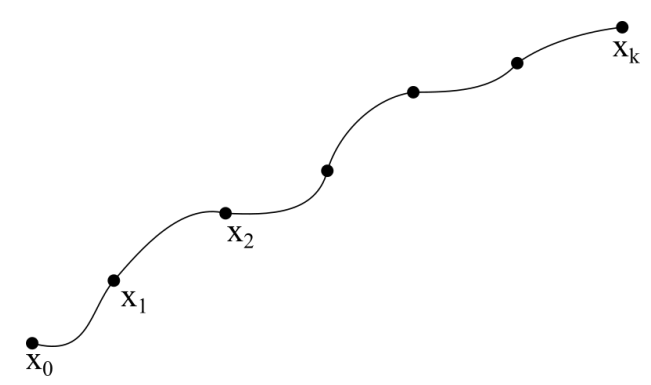

Figure 1.1: A curve

This number is then called the length of the curve $c$, denoted by length $(c)$ (see [BH99], Definition 1.18, page 12). A metric space $(X, d)$ is called a length space if $\forall x, y \in X$ there is a rectifiable curve between $x$ and $y$, and:

$$
d(x, y)=\inf \{\operatorname{length}(c) ; c(0)=x, c(1)=y\} .
$$


A geodesic segment is a map

$$
c: I \longrightarrow X,
$$

for $I \subset \mathbf{R}$ an interval, which is an isometric embedding, i.e.

$$
\forall s, t \in I, \quad d(c(s), c(t))=|t-s| .
$$

A length space $(X, d)$ is said to be a geodesic space if any two points in $X$ can be joined by at least one geodesic segment.

Remarks 1.1. i) Spaces with few or no rectifiable curves are of some interest. They, for example, appear as boundary at infinity of negatively curved manifolds when the curvature is not constant.

ii) An easy but important example is a metric graph. We could, for example, decide that the edges are isometric to $[0,1]$.

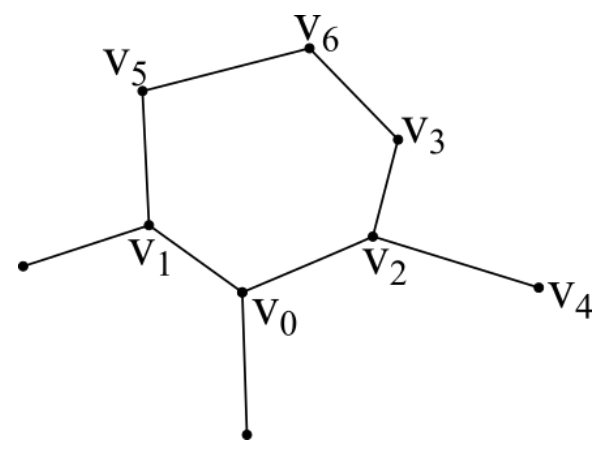

Figure 1.2: metric graph

On figure 1.2 there are two geodesics between $v_{0}$ and $v_{6}$.

\subsection{Gromov-hyperbolic spaces}

The main references that we use and suggest are [CDP90] and [BH99]. The main idea in this subsection is not to use curvature to prove comparison theorems on triangles, à la Toponogov, but to use triangle comparison to define a weak notion of sectional curvature bounded above.

We consider a proper geodesic space $X$ and a triangle $\Delta \subset X$. A triangle is the union of three points, $a, b$ and $c$, and a choice of a (minimising) geodesic between each couple of them. We call $\alpha$ (resp. $\beta, \gamma$ ) the length of the side opposite to $a$ (resp. $b, c$ ).

Because $\alpha, \beta$ and $\gamma$ satisfy the triangle inequalities there exists a Euclidean triangle $\bar{\Delta} \subset \mathbf{R}^{2}$ with the same side lengths. We call it the comparison triangle of $\Delta$.

Exercise 1.2. Prove the existence of $\bar{\Delta}$.

Now $\bar{\Delta} \subset \mathbf{R}^{2}$ has an inscribed circle and we get a map $\bar{f}: \bar{\Delta} \longrightarrow T^{*}$, onto a tripod which is an isometry when restricted to each side of $\bar{\Delta}$, see Figure 1.4 below. 

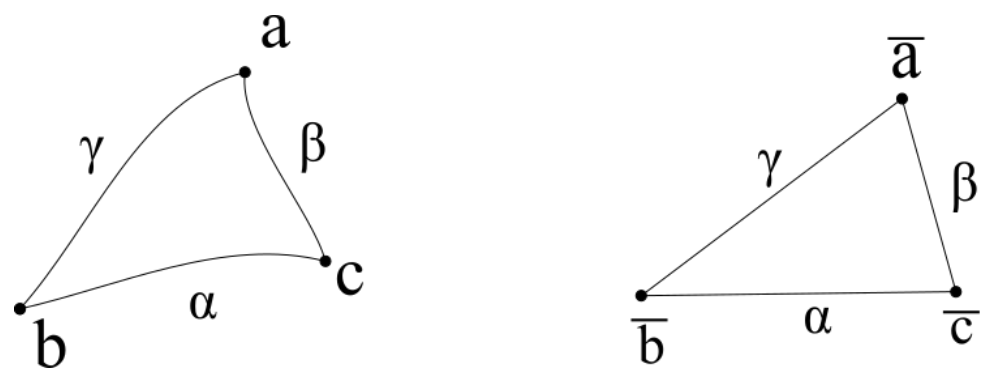

Figure 1.3: $\Delta \subset X$ and $\bar{\Delta} \subset \mathbf{R}^{2}$
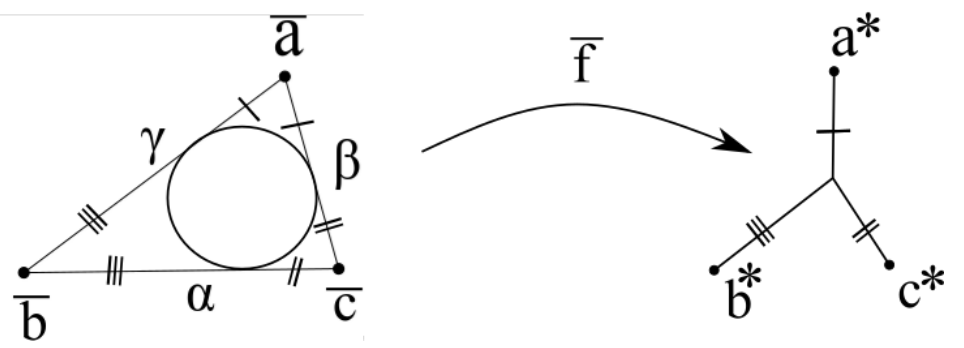

Figure 1.4: $\bar{\Delta} \subset \mathbf{R}^{2}$ and tripod $T^{*}$

Exercises 1.3. 1. Prove the equalities on the segments of $\bar{\Delta}$ shown in the figure 1.4.

2. Prove that, for any triangle, the associated tripod is unique.

There is also an obvious isometry between the sides of $\Delta$ and $\bar{\Delta}$ of same length. Together with $\bar{f}$ this combines into a map

$$
f_{\Delta}: \Delta \longrightarrow T^{*},
$$

which is an isometry between the segment $[a, b] \subset \Delta$ (resp. $[b, c],[c, a])$ and the segment $\left[a^{*}, b^{*}\right] \subset T^{*}\left(\right.$ resp. $\left.\left[b^{*}, c^{*}\right],\left[c^{*}, a^{*}\right]\right)$. Now let $\delta \geq 0$ be a real number, we get the

Definition 1.4. The space $(X, d)$ is said to be $\delta$-hyperbolic (in the sense of Gromov) if for all triangles $\Delta$ the following property holds. For all $x, y \in \Delta$, with $f_{\Delta}(x)=f_{\Delta}(y)$ we have,

$$
d(x, y) \leq \delta .
$$

Such triangles are said to be $\delta$-thin.

Notice that there could exist several triangles based on three points $a, b$ and $c$, and the above definition requires that all of them are $\delta$-thin. 
Examples 1.5. 1. Obviously, $\boldsymbol{R}^{2}$ endowed with the Euclidean distance is never $\delta$ hyperbolic.

2. On the other hand a tree is 0-hyperbolic; indeed, all triangles are tripods.

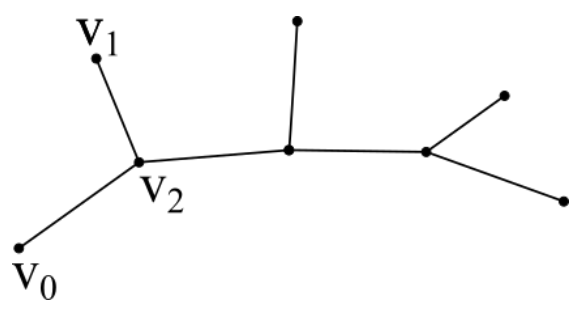

Figure 1.5: Tree, $\delta=0$.

Exercise 1.6. Prove that the hyperbolic plane is hyperbolic in the sense of Gromov and compute $\delta$.

Note that $\delta$-hyperbolicity characterizes large triangles since, by compactness, for small ones there always exist a $\delta$ satisfying the definition (we recall that the space $X$ is proper). The interpretation of Definition 1.4 is that large triangles look like $\delta$-thickened tripods. Looking at the hyperbolic plane with a metric of constant curvature equal to $\kappa<0$ we can check that, somehow, $\delta^{-2}$ behaves like $|\kappa|$. In particular an upper bound on $\delta$ can be viewed as a weak and synthetic version of an upper bound on $\kappa$; indeed, $\delta \leq C \simeq \kappa \leq-C^{-2}$.

\subsection{Measures}

We endow the metric space $(X, d)$ with a Borel measure that we assume to be nonnegative and non-trivial (i.e not identically zero). At this stage let us give the three main examples.

a) For a discrete set, possibly infinite, say $\left\{p_{1}, p_{2}, \cdots\right\}$ we shall consider the counting measure that is,

$$
\mu=\sum_{1}^{+\infty} \delta_{p_{k}}
$$

where $\delta_{p}$ is the Dirac mass at the point $p$. In the sequel the discrete set will always be the discrete orbit of a group action.

b) For a graph we shall consider the 1-dimensional Lebesgue measure supported on the edges which make them of length 1.

c) Finally, if $(X, d)$ is a Riemannian manifold $\left(M, d_{g}\right)$ (where $d_{g}$ is the distance induced by the Riemannian metric) the Riemannian measure, noted $d v_{g}$, is the most natural one. 


\subsection{Groups acting by isometries}

Let $\Gamma \subset \operatorname{Isom}(X, d)$ be a subgroup of the isometry group of the metric space $(X, d)$. We say that the action is proper if for some (hence for all) $x \in X$ we have,

$$
\forall R>0 \quad \#\{\gamma ; d(x, \gamma x) \leq R\}<+\infty .
$$

This is equivalent to $\Gamma$ being discrete in $\operatorname{Isom}(X, d)$ for the compact-open topology. We shall also consider groups $\Gamma$ which are finitely generated. We pick a finite symmetric generating set,

$$
\Sigma=\left\{\gamma_{1}, \cdots, \gamma_{n}, \gamma_{1}^{-1}, \cdots, \gamma_{n}^{-1}\right\} .
$$

To this data we associate a graph, the Cayley graph of the group, denoted by $\mathcal{G}(\Gamma, \Sigma)$. We turn this graph into a metric space, as before, by deciding that the edges are isometric to $[0,1]$. The distance then defined on $\mathcal{G}$ is noted $d_{\mathcal{G}}$. Now, if the metric space $\left(\mathcal{G}(\Gamma, \Sigma), d_{\mathcal{G}}\right)$ is $\delta$-hyperbolic for some $\delta$ we say that the group is hyperbolic in the sense of Gromov. Notice that $\delta$ then depends on $\Sigma$.

We consider the counting measure on the orbits of the action of $\Gamma$ on the space $X$, namely

$$
\mu_{x}^{\Gamma}=\sum_{\gamma \in \Gamma} \delta_{\gamma x} \quad \text { that is }, \quad \mu_{x}^{\Gamma}\left(B_{X}(x, R)\right)=\#\{\gamma ; d(x, \gamma x)<R\} .
$$

Here $B_{X}(x, R) \subset X$ is a ball of radius $R$ around the point $x \in X$. Finally we shall consider co-compact actions, that is, actions for which the topological space $X / \Gamma$ is compact. When $(X, d)$ is a metric space on which a discrete group $\Gamma$ acts by isometries we shall call $\bar{d}$ the distance on $X / \Gamma$.

\subsection{Entropy of $(X, d, \mu)$}

For a metric space with a measure as described in Subsection 1.4 we define the following well-known quantity,

$$
\operatorname{Ent}(X, d, \mu)=\lim _{R \rightarrow+\infty} \frac{1}{R} \log (\mu(B(x, R))) .
$$

The name "Entropy" comes from the dynamical nature of this invariant in the case of Riemannian manifolds.

Exercise 1.7. 1. Prove that it does not depend on $x$,

2. and that the $\underline{\lim }$ is indeed a limit when the action is co-compact.

If $\mu=\mu_{y}^{\Gamma}$ for some $y \in X$, by abuse of language, we shall denote its entropy by $\operatorname{Ent}(X, d)$. 


\subsection{Bishop-Gromov's Inequality}

This is a major comparison theorem that has been our guideline in [BCGS20b, BCGS20a]. Let us recall the Riemannian case. We consider a complete Riemannian manifold $(M, g)$ whose Ricci curvature satisfies,

$$
\text { Ric } \geq(n-1) \kappa g, \quad \text { for } \kappa \in \mathbf{R} .
$$

We can now compare the volumes of the balls around a point $m \in M$ with the volumes of balls of the same radius in a simply-connected Riemannian manifold of constant sectional curvature equal to $\kappa$ denoted by $M_{\kappa}$. More precisely, let $R>0$ be a real number, the classical Bishop's inequality is:

$$
\operatorname{Vol}\left(B_{M}(m, R)\right) \leq \operatorname{Vol}\left(B_{M_{\kappa}}(R)\right) .
$$

Let us define the function

$$
\varphi(R)=\frac{\operatorname{Vol}\left(B_{M}(m, R)\right)}{\operatorname{Vol}\left(B_{M_{\kappa}}(R)\right)},
$$

Bishop's Inequality can then be rewritten: for any $R>0, \varphi(R) \leq 1$. The improvement by Gromov is then that the function $\varphi$ is non increasing, that is, if $0<r \leq R$,

$$
\varphi(R)=\frac{\operatorname{Vol}\left(B_{M}(m, R)\right)}{\operatorname{Vol}\left(B_{M_{\kappa}}(R)\right)} \leq \frac{\operatorname{Vol}\left(B_{M}(m, r)\right)}{\operatorname{Vol}\left(B_{M_{\kappa}}(r)\right)}=\varphi(r) .
$$

Notice that volumes of balls in $M_{\kappa}$ are explicitly computable.

An interesting and obvious consequence is the following. If $(M, g)$ is a Riemannian manifold as above, say with $\kappa \leq 0$, Bishop's Inequality yields,

$$
\operatorname{Ent}\left(M, d_{g}, d v_{g}\right) \leq(n-1)|\kappa|=\operatorname{Ent}\left(M_{\kappa}\right) .
$$

Here, by abuse of language, $\operatorname{Ent}\left(M_{\kappa}\right)$ is computed with the distance and the volume element coming from the constant curvature metric. When $\kappa>0$ both $M$ and $M_{\kappa}$ are closed manifolds, their entropies are equal to zero and the above inequality is trivial. On the contrary it is particularly interesting when $(M, g)$ is the universal cover of a closed Riemannian manifold and $g$ is the pulled-back metric on $M$ and $\kappa \leq 0$.

The conclusion that is our guideline is that any upper bound on the entropy could play the role of a (very) weak and asymptotic version of a lower bound on the Ricci curvature. Although the Ricci curvature may not be defined on a metric space, the entropy exists for a metric space with mild assumptions (the one we described). We then wish to study the consequences of this weak and synthetic "Ricci curvature bounded below" condition.

\subsection{Main Theorem}

We now state the main theorem of the first part of this course. 
Theorem 1.8. Let $(X, d)$ be a $\delta$-hyperbolic metric space, $\Gamma \subset \operatorname{Isom}(X, d)$ a discrete group (acting properly) such that

$$
\operatorname{diam}(X / \Gamma, \bar{d}) \leq D \quad \text { and } \quad \operatorname{Ent}(X, d) \leq H .
$$

Then, for every $x \in X$ and $10(D+2 \delta) \leq r \leq R<+\infty$,

$$
\frac{\#\{\gamma ; d(x, \gamma x)<R\}}{\#\{\gamma ; d(x, \gamma x)<r\}}=\frac{\mu_{x}^{\Gamma}\left(B_{X}(x, R)\right)}{\mu_{x}^{\Gamma}\left(B_{X}(x, r)\right)} \leq 3\left(\frac{R}{r}\right)^{25 / 4} e^{6 H R} .
$$

Remarks 1.9. $\quad$ i) This inequality does note give any information about small balls, which is natural since neither the $\delta$-hyperbolicity nor the assumption on the entropy "see" the geometry at small scale.

ii) Furthermore it is by no means optimal.

iii) However it is a strong Bishop-Gromov inequality obtained without any pointwise assumption on the curvature, which may not exist in this context. Only large scale assumptions are required.

\section{Lecture 2}

In this lecture we intend to sketch the proof of Theorem 1.8 which will be restated below.

\subsection{Quadrangle inequality for $\delta$-hyperbolic metric spaces}

We need another characterisation of $\delta$-hyperbolicity. Let us consider a proper geodesic metric space $(X, d)$ which is furthermore $\delta$-hyperbolic and let $x, y, z$ and $w$ be four points in $X$. If we choose a geodesic between any two of them we get a quadrangle with its diagonals as shown on figure 2.1 below.

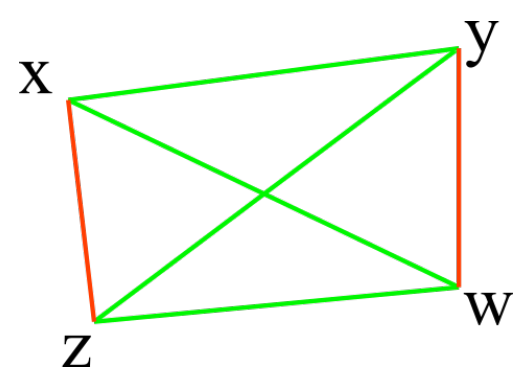

Figure 2.1: Quadrangle

The quadrangle inequality is the following,

$$
d(x, z)+d(y, w) \leq \operatorname{Max}\{d(x, y)+d(z, w), d(x, w)+d(y, z)\}+2 \delta .
$$

Exercise 2.1. Prove that this inequality is equivalent to hyperbolicity in the sense of Gromov (see [BH99] p. 410). 


\subsection{A simple version of Theorem 1.8}

Let us now state a weaker but simpler version of Theorem 1.8 which we prove below. The Main theorem follows from it by iteration, see the details in [BCGS20b].

Theorem 2.2. Let $(X, d)$ be a $\delta$-hyperbolic metric space and $\Gamma \subset \operatorname{Isom}(X, d)$ a discrete group acting properly such that

$$
\operatorname{diam}(X / \Gamma, \bar{d}) \leq D \quad \text { and } \quad \operatorname{Ent}(X, d) \leq H .
$$

Then, for every $x \in X$ and $R \geq 12(D+2 \delta)$,

$$
\frac{\mu_{x}^{\Gamma}(B(x, R))}{\mu_{x}^{\Gamma}\left(B\left(x, \frac{5}{6} R\right)\right)} \leq 1+2 e^{H R}
$$

We start with several (not too) technical lemmas.

\subsubsection{Easy lemma}

Let $\mu$ be a Borel measure on $X$, finite on compact sets, and $\Omega \subset X$ a measurable set. We then have,

$$
\int_{\Omega} \mu(B(y, r)) d \mu(y)=\int_{X} \mu(B(z, r) \cap \Omega) d \mu(z) .
$$

Exercise 2.3. Prove this inequality. Hint: this is an easy consequence of Fubini's Theorem and of the symmetry of the distance $d$.

\subsubsection{More technical lemmas}

The main lemma of this proof, which translates the $\delta$-hyperbolicity, is the following.

Lemma 2.4. Let $(X, d)$ be a $\delta$-hyperbolic metric space. $\forall R, R^{\prime} \in(0,+\infty), \forall x, x^{\prime} \in X$ such that $d\left(x, x^{\prime}\right)<R+R^{\prime}, \exists y \in X$ such that,

$$
\begin{aligned}
B(x, R) \cap B\left(x^{\prime}, R^{\prime}\right) \subset B(y, r), & \text { with } r=\operatorname{Min}\left\{R, R^{\prime}, \frac{1}{2}\left(R+R^{\prime}-d\left(x, x^{\prime}\right)\right)+2 \delta\right\}
\end{aligned}
$$

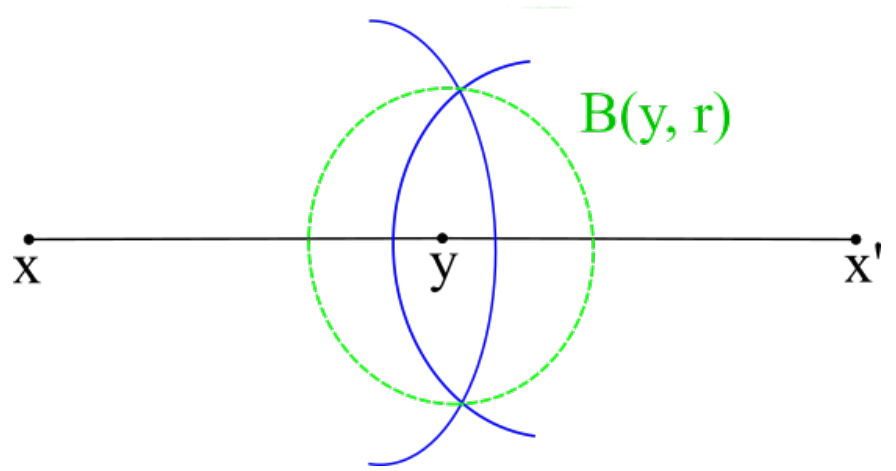

Figure 2.2: Intersection of balls 
On the figure 2.2, we have $R=R^{\prime}$ and $d(x, y)=d\left(y, x^{\prime}\right)=\frac{1}{2} d\left(x, x^{\prime}\right)$. Hence $d\left(x, x^{\prime}\right)<2 R$ and $d(y, \partial B(x, R))=R-\frac{d\left(x, x^{\prime}\right)}{2}=\frac{1}{2}\left(2 R-d\left(x, x^{\prime}\right)\right)$. The proof of Lemma 2.4 relies on the quadrangle inequality (see [BCGS20b], Lemma 8.4).

Exercises 2.5. 1. Show that this is not true in $\boldsymbol{R}^{2}(\delta=+\infty)$.

2. Show that this is trivially true on a tree $(\delta=0)$.

We now assume that $(X, d)$ satisfies the hypotheses of 2.2 . We also assume that $\mu$ is $\Gamma$-invariant. We then have,

Lemma 2.6. For every $R$ and $R^{\prime}$ such that $8 \delta \leq R^{\prime} \leq R$,

$\int_{B(x, R) \backslash B\left(x, R-\frac{1}{2} R^{\prime}\right)} \mu\left(B\left(x^{\prime}, R^{\prime}\right)\right) d \mu\left(x^{\prime}\right) \leq \mu\left(B\left(x, R+R^{\prime}\right) \backslash B\left(x, R-\frac{1}{2} R^{\prime}\right)\right) \mu\left(B\left(x, \frac{3}{4} R^{\prime}+2 \delta+D\right)\right)$.

Proof of Lemma 2.6. We shall only need, for the main theorem, to consider the measure $\mu=\mu_{x}^{\Gamma}$; however the proof of this lemma is valid when $\mu$ satisfies the above hypotheses.

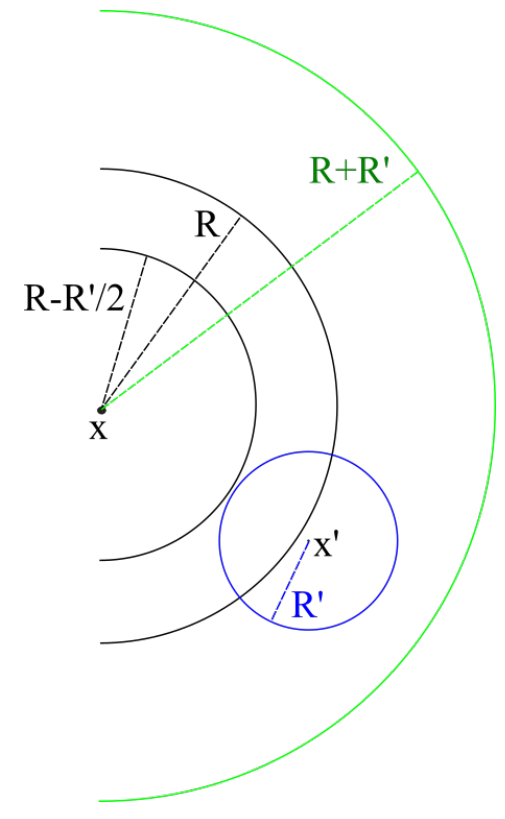

Figure 2.3:

We have,

$$
\begin{aligned}
I & :=\int_{B(x, R) \backslash B\left(x, R-\frac{1}{2} R^{\prime}\right)} \mu\left(B\left(x^{\prime}, R^{\prime}\right)\right) d \mu\left(x^{\prime}\right) \\
& =\int_{\left(B\left(x, R+R^{\prime}\right) \backslash B\left(x, R-\frac{1}{2} R^{\prime}\right)\right) \cap B(x, R)} \mu\left(B\left(x^{\prime}, R^{\prime}\right)\right) d \mu\left(x^{\prime}\right) .
\end{aligned}
$$


By lemma 2.2.1 we get,

$$
I=\int_{B\left(x, R+R^{\prime}\right) \backslash B\left(x, R-\frac{1}{2} R^{\prime}\right)} \mu\left(B\left(x^{\prime}, R^{\prime}\right) \cap B(x, R)\right) d \mu\left(x^{\prime}\right) .
$$

Let $x^{\prime} \in B\left(x, R+R^{\prime}\right) \backslash B\left(x, R-\frac{1}{2} R^{\prime}\right)$ (as in Figure 2.3), we have

$$
R-R^{\prime}+4 \delta \leq R-R^{\prime}+\frac{1}{2} R^{\prime}=R-\frac{1}{2} R^{\prime} \leq d\left(x, x^{\prime}\right)<R+R^{\prime} .
$$

We can then apply Lemma 2.4 to get that there exists $y$ such that,

$$
B\left(x^{\prime}, R^{\prime}\right) \cap B(x, R) \subset B(y, r),
$$

with

$$
r=\operatorname{Min}\left\{R, R^{\prime}, \frac{1}{2}\left(R+R^{\prime}-d\left(x, x^{\prime}\right)\right)+2 \delta\right\}=\frac{1}{2}\left(R+R^{\prime}-d\left(x, x^{\prime}\right)\right)+2 \delta \leq \frac{3}{4} R^{\prime}+2 \delta .
$$

It only remains to include the ball $B(y, r)$ into a ball centred at $x$. Since the group acts co-compactly with a quotient whose diameter is bounded above by $D$, by assumption, there exists $\gamma \in \Gamma$ such that $d(y, \gamma x) \leq D$. Hence,

$\mu\left(B\left(x^{\prime}, R^{\prime}\right) \cap B(x, R)\right) \leq \mu\left(B\left(y, \frac{3}{4} R^{\prime}+2 \delta\right)\right) \leq \mu\left(B\left(\gamma x, \frac{3}{4} R^{\prime}+D+2 \delta\right)\right)=\mu\left(B\left(x, \frac{3}{4} R^{\prime}+D+2 \delta\right)\right)$,

the last equality coming from the invariance of the measure. We finally get the desired result,

$$
I \leq \mu\left(B\left(x, \frac{3}{4} R^{\prime}+D+2 \delta\right)\right) \int_{\left(B\left(x, R+R^{\prime}\right) \backslash B\left(x, R-\frac{1}{2} R^{\prime}\right)\right)} d \mu\left(x^{\prime}\right) .
$$

Starting now we specify the measure $\mu$ to be $\mu_{x}^{\Gamma}$. We however keep the notation $\mu$ for the sake of simplicity.

Lemma 2.7. Under the same hypotheses, $\forall x \in X, \forall R, R^{\prime}$ such that $12(D+2 \delta) \leq R^{\prime} \leq$ $R$, we have

$$
\frac{\mu\left(B\left(x, R^{\prime}\right)\right)}{\mu\left(B\left(x, 5 R^{\prime} / 6\right)\right)} \leq \frac{\mu\left(B\left(x, R+R^{\prime}\right)\right)-\mu\left(B\left(x, R-R^{\prime} / 2\right)\right)}{\mu(B(x, R))-\mu\left(B\left(x, R-R^{\prime} / 2\right)\right)} .
$$

Exercise 2.8. Show that these ratios are well-defined, i.e. that the denominators are not zero. Hint: the measure is $\mu_{x}^{\Gamma}$ hence it counts the number of points of the orbit $\Gamma x$ in the balls, in particular it takes integer values. Now the fact that the denominator on the left hand side is positive is obvious (explain why); for the right hand side it requires a short proof. 
Proof of Lemma 2.7. We recall that $\mu=\mu_{x}^{\Gamma}$, hence, if $y \in \Gamma x$,

$$
\mu\left(B\left(y, R^{\prime}\right)\right)=\mu\left(B\left(x, R^{\prime}\right)\right)
$$

by invariance of $\mu$ under the action of $\Gamma$. We then get

$$
\begin{aligned}
\mu\left(B\left(x, R^{\prime}\right)\right)\left(\mu(B(x, R))-\mu\left(B\left(x, R-R^{\prime} / 2\right)\right)\right) & =\mu\left(B\left(x, R^{\prime}\right)\right) \mu\left(B(x, R) \backslash B\left(x, R-R^{\prime} / 2\right)\right) \\
& =\int_{B(x, R) \backslash B\left(x, R-R^{\prime} / 2\right)} \mu\left(\left(B\left(x, R^{\prime}\right)\right) d \mu(y)\right. \\
& =\int_{B(x, R) \backslash B\left(x, R-R^{\prime} / 2\right)} \mu\left(\left(B\left(y, R^{\prime}\right)\right) d \mu(y) .\right.
\end{aligned}
$$

The last equality comes from the fact that the only $y$ 's weighted by the measure are the points in $\Gamma x$. For simplicity we shall denote by $N$ the quantity

$$
N=\mu\left(B\left(x, R^{\prime}\right)\right)\left(\mu(B(x, R))-\mu\left(B\left(x, R-R^{\prime} / 2\right)\right)\right) .
$$

Thanks to Lemma 2.6 we then obtain,

$$
\begin{aligned}
N & \leq \mu\left(B\left(x, R+R^{\prime}\right) \backslash B\left(x, R-R^{\prime} / 2\right)\right) \mu\left(B\left(x, 3 R^{\prime} / 4+2 \delta+D\right)\right) \\
& \leq \mu\left(B\left(x, R+R^{\prime}\right) \backslash B\left(x, R-R^{\prime} / 2\right)\right) \mu\left(B\left(x, 3 R^{\prime} / 4+R^{\prime} / 12\right)\right) \\
& =\mu\left(B\left(x, R+R^{\prime}\right) \backslash B\left(x, R-R^{\prime} / 2\right)\right) \mu\left(B\left(x, 5 R^{\prime} / 6\right)\right) .
\end{aligned}
$$

Which proves Lemma 2.7 .

\subsubsection{Proof of Theorem 2.2}

We now proceed to the proof of Theorem 2.2.

Proof of Theorem 2.2. Once more we recall that $\mu=\mu_{x}^{\Gamma}$ for some $x \in X$. We choose $R^{\prime} \geq 12(D+2 \delta)$ and define $R=k R^{\prime} / 2$ for $k$ a positive integer. From Lemma 2.7 we obtain

$$
\frac{\mu\left(B\left(x, R^{\prime}\right)\right)}{\mu\left(B\left(x, 5 R^{\prime} / 6\right)\right)} \leq \frac{\mu\left(B\left(x, \frac{k+2}{2} R^{\prime}\right)\right)-\mu\left(B\left(x, \frac{k-1}{2} R^{\prime}\right)\right)}{\mu\left(B\left(x, \frac{k}{2} R^{\prime}\right)\right)-\mu\left(B\left(x, \frac{k-1}{2} R^{\prime}\right)\right)} .
$$

We now set

$$
C=\frac{\mu\left(B\left(x, R^{\prime}\right)\right)}{\mu\left(B\left(x, 5 R^{\prime} / 6\right)\right)}-1 \quad \text { and } \quad a_{k}=\mu\left(B\left(x, \frac{k}{2} R^{\prime}\right)\right),
$$

Equation (1) then yields,

$$
a_{k+2}-a_{k-1} \geq(C+1)\left(a_{k}-a_{k-1}\right) \Longrightarrow a_{k+2}-a_{k} \geq C\left(a_{k}-a_{k-1}\right) .
$$

We add these inequalities for all integers $k$ between 2 and $n$ to obtain,

$$
a_{n+2}+a_{n+1}-a_{3}-a_{2} \geq C\left(a_{n}-a_{1}\right),
$$


and because the sequence $a_{k}$ is non decreasing in $k$

$$
2\left(a_{n+2}-a_{1}\right) \geq C\left(a_{n}-a_{1}\right) \Longrightarrow a_{n+2} \geq \frac{C}{2}\left(a_{n}-a_{1}\right)+a_{1} .
$$

Iterating this inequality leads to

$$
a_{2 n} \geq\left(\frac{C}{2}\right)^{n-1}\left(a_{2}-a_{1}\right)+a_{1}
$$

Exercise 2.9. Show that $C$ and $a_{2}-a_{1}$ are positive (see Exercise 2.8).

Finally, we can now use the second assumption on the metric space $(X, d)$, that is, $\operatorname{Ent}(X, d) \leq H$. More precisely,

$$
H \geq \operatorname{Ent}(X, d)=\lim _{n \rightarrow+\infty}\left(\frac{1}{n R^{\prime}} \log \mu\left(B\left(x, n R^{\prime}\right)\right)\right)=\lim _{n \rightarrow+\infty} \frac{1}{n R^{\prime}} \log \left(a_{2 n}\right) \geq \frac{1}{R^{\prime}} \log \left(\frac{C}{2}\right),
$$

that is,

$$
C \leq 2 e^{H R^{\prime}} \quad \text { and } \quad 1+C=\frac{\mu\left(B\left(x, R^{\prime}\right)\right)}{\mu\left(B\left(x, 5 R^{\prime} / 6\right)\right)} \leq 1+2 e^{H R^{\prime}} .
$$

This ends the proof of Theorem 2.2.

\section{Lecture 3}

The theme of this lecture is to illustrate the relations between the geometry and the topology of a space. The idea is that given a topological space $Y$, the possible metrics on $Y$ which are compatible with the topology of $Y$ have to satisfy constraints and conversely, given a metric on $Y$, the geometrical properties of this metric provides restrictions on the topology. These questions have been extensively studied in the setting of Riemannian manifolds. Our purpose in this lecture is to consider one example where the metrics under consideration are not necessary Riemannian metrics. One important topological invariant is the fundamental group of $Y$, which is finitely generated when $Y$ is compact. The abelianisation of the fundamental group of $Y$ is an abelian group called the first homology group with integer coefficients. It is also a topological invariant of $Y$, simpler than the fundamental group.

\subsection{Fundamental group}

For "many reasonable" spaces $Y$, there exist a connected and simply-connected space $\widetilde{Y}$ and a group $\Gamma$ acting on $\widetilde{Y}$ freely and properly by homeomorphisms such that $Y=\widetilde{Y} / \Gamma$. The group $\Gamma$ is called the fundamental group of $Y$ and is denoted by $\Gamma=\pi_{1} Y$.

- The circle $Y=\mathbf{R} / \mathbf{Z}=S^{1}$ has fundamental group $\pi_{1} Y=\mathbf{Z}$. 

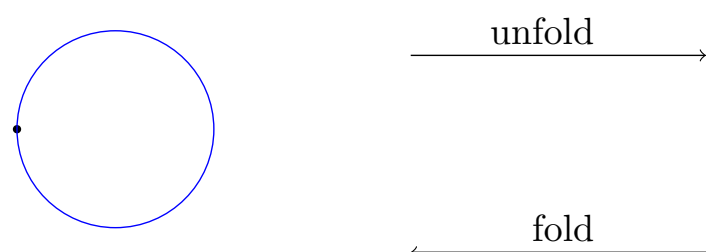

Figure 3.1: $S^{1}=\mathbf{R} / \mathbf{Z}$

- The bouquet of two circles $Y=S^{1} \vee S^{1}=T / \mathbf{F}_{2}$, where $T$ is the regular tree with valency 4 , has the non abelian free group on 2 generators as fundamental group.
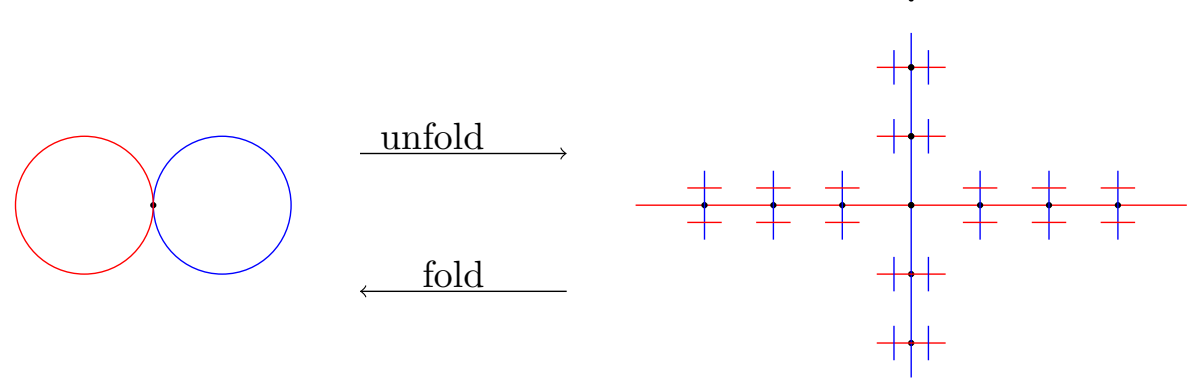

Figure 3.2: $S^{1} \vee S^{1}=T / \mathbf{F}_{2}$

\subsection{First homology group}

The fundamental group of a space $Y$ may not be abelian. For example, when $Y$ is a bouquet of two circles, $\pi_{1} Y$ is the non abelian free group on 2 generators.

Definition 3.1. Let $Y$ be a space with fundamental group $\Gamma=\pi_{1} Y$. The first homology group of $Y$ with integral coefficients is defined as the abelianised group $\Gamma /[\Gamma, \Gamma]$. It is denoted by $H_{1}(Y, \mathbf{Z})$.

Recall that the commutator subgroup $[\Gamma, \Gamma]$ of $\Gamma$ is the subgroup of $\Gamma$ generated by the set of "commutators" $\left\{a b a^{-1} b^{-1} \mid a, b \in \Gamma\right\}$. The homology group $H_{1}(Y, \mathbf{Z})=\Gamma /[\Gamma, \Gamma]$ is by definition abelian. When the space $Y$ is compact, $\Gamma=\pi_{1} Y$ is finitely generated and so is $H_{1}(Y, \mathbf{Z})$, therefore by the fundamental Theorem for finitely generated abelian groups,

$$
H_{1}(Y, \mathbf{Z}) \cong \mathbf{Z}^{r} \times \mathbf{Z} / n_{1} \mathbf{Z} \times \cdots \times \mathbf{Z} / n_{k} \mathbf{Z},
$$


where $r$ and the $n_{i}$ 's are integers, $r \geq 0$ and $n_{i} \geq 2$. In this decomposition, the factor $\mathbf{Z} / n_{1} \mathbf{Z} \times \cdots \times \mathbf{Z} / n_{k} \mathbf{Z}$ [resp. $\mathbf{Z}^{r}$ ] is the torsion [resp. torsion free] part of the homology group $H_{1}(Y, \mathbf{Z})$. Notice that when $k=0$, there is no torsion part of $H_{1}(Y, \mathbf{Z})$ and similarly, there is no torsion free part when $r=0$.

Definition 3.2. Let $Y$ be a compact space and $H_{1}(Y, \mathbf{Z}) \cong \mathbf{Z}^{r} \times \mathbf{Z} / n_{1} \mathbf{Z} \times \cdots \times \mathbf{Z} / n_{k} \mathbf{Z}$ the first homology group of $Y$. The first Betti number of $Y$ is defined as $b_{1}(Y):=r$.

Examples 3.3. 1) $H_{1}\left(S^{1}, \mathbf{Z}\right) \cong \mathbf{Z}$ and $H_{1}\left(S^{1} \times S^{1}, \mathbf{Z}\right) \cong \mathbf{Z}^{2}$

2) $H_{1}\left(S^{1} \vee S^{1}, \mathbf{Z}\right) \cong \mathbf{Z}^{2}$

3) $H_{1}(\mathrm{Nil}, \mathbf{Z}) \cong \mathbf{Z}^{2}$, where Nil $:=\operatorname{Heis}(\mathbf{R}) / \operatorname{Heis}(\mathbf{Z})$ with

$$
\operatorname{Heis}(\mathbf{A})=\left\{\left(\begin{array}{ccc}
1 & x & z \\
0 & 1 & y \\
0 & 0 & 1
\end{array}\right) / \mathrm{x}, \mathrm{y}, \mathrm{z} \in \mathbf{A}\right\}
$$

\subsection{Bounding the first Betti number}

The following result is emblematic of the constraints imposed by geometry to topology.

Theorem 3.4 ([Gro07], [Gal83b],[Gal83a]). There exists a constant $C(\Lambda, n, D)>0$ such that for every closed $n$-dimensional Riemannian manifold $\left(M^{n}, g\right)$ with Ricci curvature satisfying Ric $_{g} \geq-\Lambda g$ and $\operatorname{diam}(M, g) \leq D$, then $b_{1}(M) \leq C(\Lambda, n, D)$.

The following examples show that the assumptions on the curvature and the diameter are necessary assumptions in Theorem 3.4.

Examples 3.5. 1) Let $\Sigma_{g}$ be a hyperbolic surface of genus $g$. The fundamental group $\Gamma$ of $\Sigma_{g}$ has the following presentation, $\Gamma=<a_{1}, b_{1}, \cdots, a_{g}, b_{g} \mid \Pi_{i=1}^{g}\left[a_{i}, b_{i}\right]=1>$, hence $H_{1}\left(\Sigma_{g}, \mathbf{Z}\right)=\mathbf{Z}^{2 g}$ and the first Betti number of $\Sigma_{g}$ is $b_{1}\left(\Sigma_{g}\right)=2 g$. Notice that the diameter of $\Sigma_{g}$ satisfies $D:=\operatorname{diam} \Sigma_{g} \geq \log (2 g-1)$. This follows from the fact that the area of a genus $g$ hyperbolic surface satisfies $\operatorname{Vol} \Sigma_{g}=4 \pi(g-1) \leq \operatorname{Vol} B(D)=$ $2 \pi(\cosh D-1)$, where $B(D)$ is the hyperbolic ball of radius $D$. In particular, the surfaces $\Sigma_{g}$ have constant curvature $\kappa \equiv-1$, diameter and first Betti number tending to infinity with $g$. 


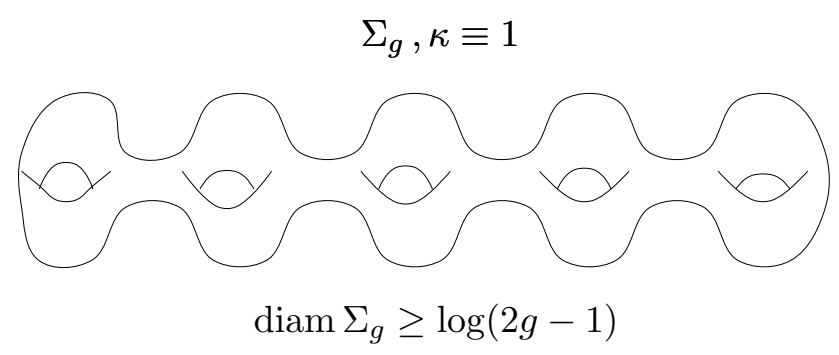

Figure 3.3: Large diameter and Betti number

2) In the preceeding example, the curvature of $\Sigma_{g}$ is bounded and the diameter and the Betti number tend to infinity with $g$. Contracting the hyperbolic metric on $\Sigma_{g}$ so that the diameter is equal to 1 , the Betti number does not change while the curvature tends to $-\infty$. This second example shows that a lower bound on the curvature is necessary in order to get a bound on the first Betti number.

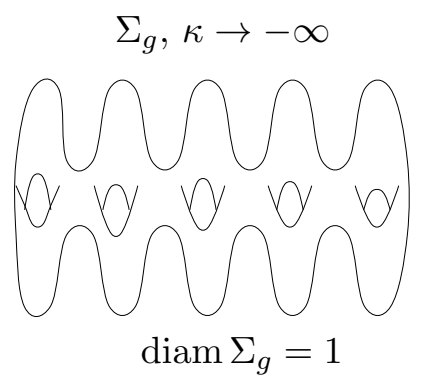

Figure 3.4: Unbounded curvature and large Betti number

The next Theorem deals with Gromov hyperbolic metric spaces. We obtain a bound on the first Betti number with an upper bound on the entropy instead of a lower bound on the curvature, The entropy assumption is much weaker than the curvature one.

Theorem 3.6. Let $(Y, d)$ be a compact, arcwise connected metric space. We suppose that the universal cover $(\widetilde{Y}, \tilde{d})$ is $\delta$-hyperbolic and that

$$
\operatorname{Ent}(\tilde{Y}, \tilde{d}) \leq H \text { and } \operatorname{diam}(Y, d) \leq D .
$$


Then

$$
b_{1}(Y) \leq 4^{8} \cdot e^{24 H(8 D+15 \delta)} .
$$

Proof. The proof follows an argument of M. Gromov and relies on a packing argument. Let us denote by $\Gamma$ the fundamental group of $(Y, d)$. Let us recall that $H_{1}(Y, \mathbf{Z})=\Gamma /$ $[\Gamma, \Gamma]$. We first notice that the torsion free part $\mathbf{Z}^{r}$ of $H_{1}(Y, \mathbf{Z}) \cong \mathbf{Z}^{r} \times \mathbf{Z} / n_{1} \mathbf{Z} \times \cdots \times \mathbf{Z} / n_{k} \mathbf{Z}$ can be seen as a lattice in $\mathbf{R}^{r}$. From now on we will assume $r \geq 1$ since Theorem 3.6 is trivially true if $r=0$. We define the two following projection maps, $p_{1}: \Gamma \rightarrow \Gamma /[\Gamma, \Gamma]$ and $p_{2}: \Gamma /[\Gamma, \Gamma] \cong \mathbf{Z}^{r} \times \mathbf{Z} / n_{1} \mathbf{Z} \times \cdots \times \mathbf{Z} / n_{k} \mathbf{Z} \rightarrow \mathbf{Z}^{r}$. We now consider $p:=p_{2} \circ p_{1}: \Gamma \rightarrow \mathbf{Z}^{r}$ and $\phi:=\iota \circ p: \Gamma \rightarrow \mathbf{R}^{r}$, where $\iota: \mathbf{Z}^{r} \hookrightarrow \mathbf{R}^{r}$ is the natural inclusion.

We now observe that the set $p(S)$ is a generating set of the group $\mathbf{Z}^{r}$ since $p$ is a surjective morphism. This implies that $\phi(S)$ generates $\mathbf{R}^{r}$ as a vector space, therefore $r \leq \# S$. Hence, we see that the first Betti number $b_{1}(Y)=r$ of $Y$ can be estimated by the cardinality of any generating set of the fundamental group $\Gamma$ of $Y$. Unfortunately, there are no upper bounds of the cardinality of generating sets of $\Gamma$; however the following lemma will allow us to replace $\Gamma$ by a finite index subgroup $\Gamma^{\prime}$ with a generating set of bounded cardinality.

Lemma 3.7. Let $S^{\prime}$ be a finite subset of $\Gamma$ generating a finite index subgroup $\Gamma^{\prime} \leq \Gamma$. Then, the set $\phi\left(S^{\prime}\right)$ generates $\mathbf{R}^{r}$ as a vector space. In particular, $r \leq \# S^{\prime}$.

Proof. Let $V \subset \mathbf{R}^{r}$ be the vector space generated by $\phi\left(S^{\prime}\right)$. We want to show that $V=\mathbf{R}^{r}$. Since the index of $\Gamma^{\prime}$ in $\Gamma$ is finite, the subset $\left\{\gamma^{k} \Gamma^{\prime}\right\}_{k \in \mathbf{Z}} \subset \Gamma / \Gamma^{\prime}$ is finite for every $\gamma \in \Gamma$. Notice that the quotient $\Gamma / \Gamma^{\prime}$ may not be a group and that it is identified with the set of right equivalence classes $\left\{\gamma \Gamma^{\prime}\right\}_{\gamma \in \Gamma}$. Therefore, there exist $k \neq l \in \mathbf{Z}$ such that $\gamma^{k} \Gamma^{\prime}=\gamma^{l} \Gamma^{\prime}$, hence $\gamma^{k-l}=\gamma^{\prime} \in \Gamma^{\prime}$ and $\phi(\gamma)=(k-l)^{-1} \phi\left(\gamma^{\prime}\right)$. This implies that $V$ contains the vector space generated by $\phi(S)$, which is $\mathbf{R}^{r}$.

The proof of Theorem 3.6 therefore boils down to finding a finite subset $S^{\prime}$ of $\Gamma$ generating a finite index subgroup $\Gamma^{\prime}$ of $\Gamma$ such that

$$
\# S^{\prime} \leq 4^{8} \cdot e^{24 H(8 D+15 \delta)} .
$$

In order to construct the set $S^{\prime}$, we proceed as follows. The idea is that the set $S^{\prime}$ has to be sufficiently dense to insure that the group $\Gamma^{\prime}$ generated by $S^{\prime}$ satisfies $\# \Gamma /$ $\Gamma^{\prime}<\infty$ but sufficiently sparse so that its cardinality stays bounded above. This will be a consequence of our Bishop-Gromov estimate 1.8.

We pick up a point $x \in \widetilde{Y}$. Let $R>0$ and $S^{\prime}:=\left\{\gamma_{i}\right\}_{i \in I}$ be a subset of $\Gamma$ of maximal cardinality such that

(i) $d\left(x, \gamma_{i}(x)\right) \leq 2 D+R$

(ii) $d\left(\gamma_{i}(x), \gamma_{j}(x)\right) \geq R$, for $i \neq j$.

Let $\Gamma^{\prime}$ be the subgroup of $\Gamma$ generated by $S^{\prime}=\left\{\gamma_{i}\right\}_{i \in I}$.

Claim 3.8. $\# \Gamma / \Gamma^{\prime}<\infty$. 


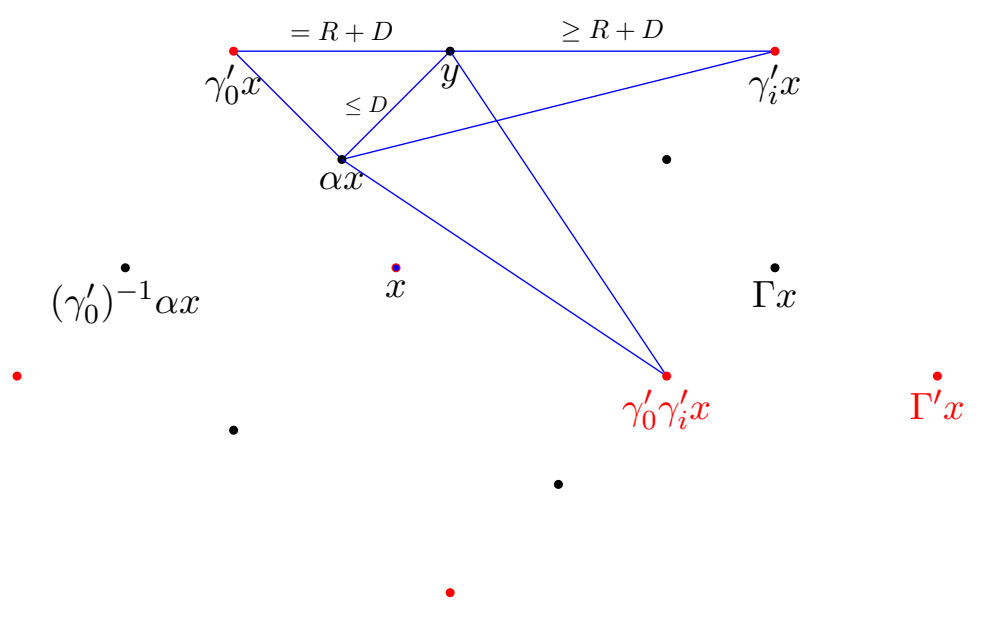

Figure 3.5: $\# \Gamma / \Gamma^{\prime}<\infty$

Proof. The claim follows from the fact that for every $y \in \tilde{Y}, d\left(y, \Gamma^{\prime} x\right)<R+D$. Indeed, if this is the case, for every $\gamma \in \Gamma$, we have $d\left(x, \gamma \Gamma^{\prime} x\right)=d\left(\gamma^{-1} x, \Gamma^{\prime} x\right)<R+D$, hence for each coset $\gamma \Gamma^{\prime}$, the orbit $\gamma \Gamma^{\prime} x$ meets $B(x, R+D)$. Therefore we have

$$
\# \Gamma / \Gamma^{\prime} \leq \#\{\gamma \in \Gamma \mid d(x, \gamma x) \leq D+R\}<\infty
$$

since $\Gamma$ acts properly discontinuously on $\tilde{Y}$. It remains to prove that for every $y \in \tilde{Y}$, $d\left(y, \Gamma^{\prime} x\right)<R+D$, see the figure 3.5 below.

Assume by contradiction that this is not the case, then there exists $y \in \widetilde{Y}$ such that $d\left(y, \Gamma^{\prime} x\right) \geq D+R$. Moving $y$ a bit if necessary, we may moreover assume that

$$
d\left(y, \Gamma^{\prime} x\right)=d\left(y, \gamma_{0}^{\prime} x\right)=D+R .
$$

Since the diameter of $Y=\widetilde{Y} / \Gamma$ satisfies $\operatorname{diam} Y \leq D$, we can choose $\alpha \in \Gamma \backslash \Gamma^{\prime}$ such that $d(y, \alpha x) \leq D$. Now, applying twice the triangle inequality gives:

$$
R \leq d\left(\left(\gamma_{0}^{\prime}\right)^{-1} \alpha x, x\right)=d\left(\alpha x, \gamma_{0}^{\prime} x\right) \leq 2 D+R .
$$

Moreover, also by the triangle inequality, we have $d\left(\alpha x, \gamma_{0}^{\prime} \gamma_{i}^{\prime} x\right) \geq d\left(\gamma_{0}^{\prime} \gamma_{i}^{\prime} x, y\right)-d(y, \alpha x)$ and since $d\left(y, \gamma_{0}^{\prime} \gamma_{i}^{\prime} x\right) \geq D+R$ and $d(y, \alpha x) \leq D$ by assumption, we get

$$
d\left(\left(\gamma_{0}^{\prime}\right)^{-1} \alpha x, \gamma_{i}^{\prime} x\right)=d\left(\alpha x, \gamma_{0}^{\prime} \gamma_{i}^{\prime} x\right) \geq R .
$$

We deduce from (2) and (3) that the set $\left.\left\{\gamma_{i}\right\}_{i \in I} \cup\left\{\left(\gamma_{0}^{\prime}\right)^{-1} \alpha\right\}\right\}$ satisfies the above properties (i) and (ii) contradicting the maximality of the cardinality of $\left\{\gamma_{i}\right\}_{i \in I}$ since $\alpha \in \Gamma \backslash \Gamma^{\prime}$. This ends the proof of the claim. 
In order to finish the proof of the Theorem 3.6 we now bound $\# S^{\prime}$. Let us consider the counting measure $\mu=\sum_{\gamma \in \Gamma} \delta_{\gamma x}$ of the $\Gamma$-orbit of $x$. Notice that by the properties (i) and (ii) defining $S^{\prime}$, for every $\gamma_{i}^{\prime} \in S^{\prime}$ we have $B\left(\gamma_{i}^{\prime} x, \frac{R}{2}\right) \subset B\left(x, 2 D+\frac{3 R}{2}\right)$ and the balls $B\left(\gamma_{i}^{\prime}, \frac{R}{2}\right)$ are disjoint. Therefore $\mu\left(B\left(x, 2 D+\frac{3 R}{2}\right)\right) \geq \# S^{\prime} \mu\left(B\left(x, \frac{R}{2}\right)\right)$, thus

$$
\# S^{\prime} \leq \frac{\mu\left(B\left(x, 2 D+\frac{3 R}{2}\right)\right)}{\mu\left(B\left(x, \frac{R}{2}\right)\right.} .
$$

We choose $R=20(D+2 \delta)$ in (4) and we apply Theorem 1.8 with $(r, R)$ replaced by $(10(D+2 \delta), 32 D+60 \delta)$. This gives

$$
\# S^{\prime} \leq 3\left(\frac{16 D+30 \delta}{5 D+10 \delta}\right)^{25 / 4} e^{24 H(8 D+15 \delta)},
$$

hence the estimate

$$
b_{1}(Y) \leq \# S^{\prime} \leq 4^{8} \cdot e^{24 H(8 D+15 \delta)}
$$

follows. This concludes the proof of Theorem 3.6.

\section{Lecture 4}

We now want to extend our version of the Bishop-Gromov inequality to balls of small radii. Using the notation of Lecture 2 we may distinguish the three following cases.

i) $R \geq r \geq 10(D+2 \delta)$ : this is the case described in Section 2 .

ii) $0<r \leq R<10(D+2 \delta)$ : this is the case that will be treated in the present section.

iii) $0<r<10(D+2 \delta) \leq R$ : this is an easy consequence of the two previous cases and its proof is left to the reader. However for the sake of completeness we state this version at the end of this section.

In the previous sections no local assumption was made on the metric space $(X, d)$. We need now to require another property in order to have some control on the local behaviour.

\subsection{Busemann Spaces}

We call normal parametrisation of a geodesic $c:[0,1] \longrightarrow X$ the parametrisation such that

$$
d(c(0), c(t))=t d(c(0), c(1)), \quad \text { for all } t>0 .
$$

Definition 4.1. A complete, proper, geodesic metric space $(X, d)$ is said to be a Busemann space if its metric $d$ is convex, i.e. for any two geodesic segments $c_{1}$ and $c_{2}$ with normal parametrisations,

$$
c_{1}, c_{2}:[0,1] \longrightarrow X \quad \text { such that } c_{1}(0)=c_{2}(0),
$$

the function $t \mapsto d\left(c_{1}(t), c_{2}(t)\right)$ is convex. 


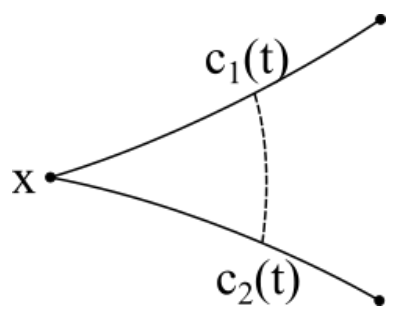

Figure 4.1: Convexity in Busemann spaces

Remarks 4.2. $\quad$ 1. On every Busemann space any two points are joined by a unique geodesic segment.

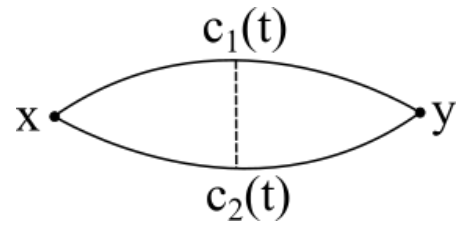

Figure 4.2: $d\left(c_{1}(t), c_{2}(t)\right)$ is not convex

2. Examples of Busemann spaces are given by simply connected manifolds of non positive sectional curvature (Cartan-Hadamard manifolds).

3. Closed geodesic balls or, more generally, closed convex domains in Cartan-Hadamard manifolds are also Busemann spaces.

We will furthermore always assume that the space $(X, d)$ satisfies the property of extension of geodesics, that is, for every geodesic segment

$$
c:[a, b] \longrightarrow X, \quad a<b
$$

there exists $\epsilon>0$ and a geodesic segment $c^{\prime}:[a, b+\epsilon] \longrightarrow X$ such that $c_{[[a, b]}^{\prime}=c$. Notice that this excludes closed convex domains with non-empty boundary in CartanHadamard manifolds. This assumption will be used in the proof of lemma 4.8.

We then state the main theorem of this section.

Theorem 4.3. Let $(X, d)$ be a $\delta$-hyperbolic and Busemann metric space, $\Gamma \subset \operatorname{Isom}(X, d)$ be a discrete group acting properly such that

$$
\operatorname{diam}(X / \Gamma, \bar{d}) \leq D \quad \text { and } \quad \operatorname{Ent}(X, d) \leq H .
$$

Then, for every $x \in X$ and $0<r \leq R<12(D+2 \delta)$,

$$
\frac{\mu_{x}^{\Gamma}(\bar{B}(x, R))}{\mu_{x}^{\Gamma}(B(x, r))} \leq C\left(\frac{R}{r}\right)^{A} \exp \left(18 H(11 D+20 \delta) \frac{R}{r}\right),
$$

here $C$ and $A$ can be explicitly estimated. More precisely $C=\frac{11}{2} \cdot 3^{25 / 4}$ and $A=25 / 4$. 
Remark 4.4. Notice that on the left hand side, at the numerator, we compute the $\mu_{x}^{\Gamma-}$ measure of the closure of the ball $B(x, R)$. This is an improvement since there might be points of the orbit of $x$ on the boundary $\bar{B}(x, R) \backslash B(x, R)$.

The proof of Theorem 4.3 relies on arguments of packing and covering big balls by smaller ones.

\subsection{Packings and Coverings}

With the above notation and assumptions, that is, $(X, d)$ is a proper geodesic metric space and $\Gamma \subset \operatorname{Isom}(X)$ is a discrete subgroup, we have the following definitions.

Definition 4.5. $\quad$ i) An r-packing of a ball $B(x, R)$ is any family $\left(B\left(y_{j}, r\right)\right)_{j \in J}$ of disjoint balls included in $B(x, R)$. We set

$\operatorname{Pack}(x, r, R)=$ the maximal number of balls in a r-packing of $B(x, R)$.

ii) $A(\Gamma x, r)$-packing of $B(x, R)$ is any family $\left(B\left(\gamma_{j} x, r\right)\right)_{j \in J}$ of disjoint balls included in $B(x, R)$. We set

$\operatorname{Pack}_{\Gamma}(x, r, R)=$ the maximal number of balls in a $(\Gamma x, r)$-packing of $B(x, R)$.

iii) $A(\Gamma x, r)$-covering of $\bar{B}(x, R)$ is any family $\left(B\left(\gamma_{j} x, r\right)\right)_{j \in J}$ of balls such that $\bar{B}(x, R) \cap$ $\Gamma x \subset \cup_{j \in J} B\left(\gamma_{j} x, r\right)$. We set

$\operatorname{Cover}_{\Gamma}(x, r, R)=$ the maximal number of balls in a $(\Gamma x, r)$-covring of $\bar{B}(x, R)$.

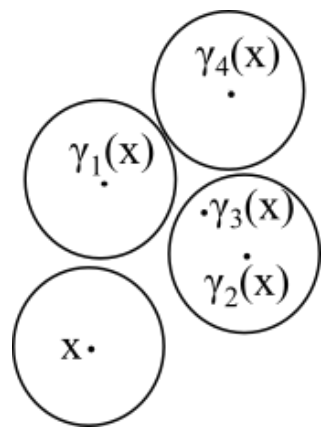

Figure 4.3: $\operatorname{Pack}_{\Gamma}(x, r, R)$

We then have the following lemma.

Lemma 4.6. Let us assume that $\Gamma$ acts co-compactly on $X$ and that the quotient space has diameter not greater than $D>0$, then one has, for every $x \in X, 0<r \leq R$,

i) $\operatorname{Cover}_{\Gamma}(x, r, R) \leq \operatorname{Pack}_{\Gamma}(x, r / 2, R+r / 2)$. 
ii) $\operatorname{Pack}_{\Gamma}(x, r, R) \leq \frac{\mu_{x}^{\Gamma}(B(x, R))}{\mu_{x}^{\Gamma}(B(x, r))} \leq \frac{\mu_{x}^{\Gamma}(\bar{B}(x, R))}{\mu_{x}^{\Gamma}(B(x, r))} \leq \operatorname{Cover}_{\Gamma}(x, r, R)$.

iii) $\operatorname{Pack}_{\Gamma}(x, r, R) \leq \operatorname{Pack}(x, r, R) \leq \operatorname{Pack}_{\Gamma}(x, r-D, R)$, the last inequality making sense only when $r>D$.

Exercise 4.7. Prove this lemma.

\subsection{A Contraction in Busemann Spaces}

We now assume that $(X, d)$ is Busemann and has the property of extension of geodesics. The second tool that we shall use essentially depends on the convexity of the distance. We construct a contraction (or a dilation) as follows. Let $x \in X, \forall y \in X$ we call $c_{y}:[0,1] \longrightarrow X$ the (unique) geodesic normally parametrised joining $x$ to $y$, that is $c_{y}(0)=x$ and $c_{y}(1)=y$.

Then, $\forall \lambda \in[0,1]$, we define

$$
\varphi_{x, \lambda}: X \longrightarrow X \quad \text { by } \quad \varphi_{x, \lambda}(y)=c_{y}(\lambda) .
$$

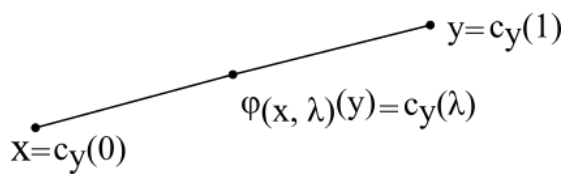

Figure 4.4: $\lambda$-contraction

Lemma 4.8. One has, $\forall y, y^{\prime} \in X, \quad d\left(\varphi_{x, \lambda}(y), \varphi_{x, \lambda}\left(y^{\prime}\right)\right) \leq \lambda d\left(y, y^{\prime}\right)$. As a consequence we get, $\forall \alpha \geq 1$,

$$
\operatorname{Pack}(x, r, R) \leq \operatorname{Pack}(x, \alpha r, \alpha R) .
$$

Proof. The first inequality follows from the convexity of the distance, indeed

$$
d\left(c_{y}(\lambda), c_{y^{\prime}}(\lambda)\right) \leq(1-\lambda) d\left(c_{y}(0), c_{y^{\prime}}(0)\right)+\lambda d\left(c_{y}(1), c_{y^{\prime}}(1)\right)=\lambda d\left(y, y^{\prime}\right) .
$$

The second inequality is left to the reader; notice that it is in the proof of this inequality on the packing numbers that the property of extendable geodesics is needed.

We are now ready to prove Theorem 4.3.

Proof of Theorem 4.3. For the sake of simplicity, let us set

$$
I=\frac{\mu_{x}^{\Gamma}(\bar{B}(x, R))}{\mu_{x}^{\Gamma}(B(x, r))} .
$$

Lemma 4.6 yields

$$
I \leq \operatorname{Cover}_{\Gamma}(x, r, R) \leq \operatorname{Pack}_{\Gamma}(x, r / 2, R+r / 2) \leq \operatorname{Pack}(x, r / 2, R+r / 2),
$$


and Lemmas 4.8 and 4.6 imply, for $\alpha \geq 1$ and $\alpha(r / 2)>D$,

$$
I \leq \operatorname{Pack}(x, \alpha r / 2, \alpha(R+r / 2)) \leq \operatorname{Pack}(x, \alpha(r / 2)-D, \alpha(R+r / 2)) .
$$

We now choose $\alpha$ so that $r^{\prime}=\alpha(r / 2)-D=10(D+2 \delta)$. This clearly implies that $R^{\prime}=\alpha(R+r / 2) \geq 10(D+2 \delta)$ and by the assumption on $r$ (in Theorem 4.3) that $\alpha \geq 1$. Finally, we can apply Theorem 1.8 and get,

$$
I \leq \operatorname{Pack}\left(x, r^{\prime}, R^{\prime}\right) \leq \frac{\mu_{x}^{\Gamma}\left(B_{X}\left(x, R^{\prime}\right)\right)}{\mu_{x}^{\Gamma}\left(B_{X}\left(x, r^{\prime}\right)\right)} \leq 3\left(\frac{R^{\prime}}{r^{\prime}}\right)^{25 / 4} e^{6 H R^{\prime}} .
$$

It then suffices to replace $R^{\prime}$ and $r^{\prime}$ by their respective values in terms of $R, r, D$ and $\delta$.

Next we prove an inequality relating the Bishop-Gromov ratio associated to a group $\Gamma$ to the Bishop-Gromov ratio associated to a subgroup $\Gamma^{\prime} \subset \Gamma$.

Corollary 4.9. Let $(X, d)$ be a proper geodesic metric space and $\Gamma \subset \operatorname{Isom}(X)$ a discrete subgroup. If $\Gamma^{\prime} \subset \Gamma$ is a subgroup of $\Gamma$ then, $\forall x \in X, \forall r, R$ such that $0<r \leq R$, one has

$$
\frac{\mu_{x}^{\Gamma^{\prime}}\left(\bar{B}_{X}(x, R)\right.}{\mu_{x}^{\Gamma^{\prime}}\left(B_{X}(x, r)\right)} \leq \frac{\mu_{x}^{\Gamma}\left(B_{X}(x, R+r / 2)\right)}{\mu_{x}^{\Gamma}\left(B_{X}(x, r / 2)\right)}
$$

Proof. From Lemma 4.6 we get

$$
\begin{aligned}
\frac{\mu_{x}^{\Gamma^{\prime}}\left(\bar{B}_{X}(x, R)\right.}{\mu_{x}^{\Gamma^{\prime}}\left(B_{X}(x, r)\right)} & \leq \operatorname{Cover}_{\Gamma^{\prime}}(x, r, R) \leq \operatorname{Pack}_{\Gamma^{\prime}}(x, r / 2, R+r / 2) \\
& \leq \operatorname{Pack}_{\Gamma}(x, r / 2, R+r / 2) \leq \frac{\mu_{x}^{\Gamma}\left(B_{X}(x, R+r / 2)\right)}{\mu_{x}^{\Gamma}\left(B_{X}(x, r / 2)\right)} .
\end{aligned}
$$

The inequality between the two packing numbers follows from the obvious remark that $\Gamma^{\prime} x \subset \Gamma x$.

As announced at the beginning of this section, we end it by stating the intermediate Bishop-Gromov's inequality for $\mu_{x}^{\Gamma}$ that is when $r$ is small and $R$ is large. More precisely,

Theorem 4.10. Let $(X, d)$ be a $\delta$-hyperbolic and Busemann metric space, $\Gamma \subset \operatorname{Isom}(X, d)$ be a discrete group acting properly such that

$$
\operatorname{diam}(X / \Gamma, \bar{d}) \leq D \quad \text { and } \quad \operatorname{Ent}(X, d) \leq H .
$$

Then, for every $x \in X$ and $0<r<12(D+2 \delta) \leq R$,

$$
\frac{\mu_{x}^{\Gamma}\left(\bar{B}_{X}(x, R)\right)}{\mu_{x}^{\Gamma}\left(B_{X}(x, r)\right)} \leq C^{\prime}\left(\frac{R}{r}\right)^{A^{\prime}} \exp \left(18 H \frac{(11 D+10 \delta)^{2}}{r}+6 H R\right),
$$

here $C^{\prime}$ and $A^{\prime}$ can be explicitly computed. More precisely, $C^{\prime}=\frac{11}{2} \cdot 3^{29 / 4}$ and $A^{\prime}=25 / 4$. 


\section{Lecture 5}

In this lecture, we derive from our Bishop-Gromov Theorem a version of the Margulis lemma in the context of cocompact discrete groups of isometries of $\delta$-hyperbolic spaces with bounded entropy. From this "metric Margulis lemma", we deduce estimates on the systole of such actions. Such estimates are classical for manifolds of bounded non positive curvature.

\subsection{Growth of groups}

Let $\Gamma$ be a group and $S$ a finite set of generators of $\Gamma$. We denote by $\left(\mathcal{G}(\Gamma, S), d_{S}\right)$ the Cayley graph of $\Gamma$ with the word distance $d_{S}$ associated to $S$ and for $k \in \mathbf{N}, B_{S}(k)$ the ball of radius $k$ centered at $e$ in the Cayley graph. We also define the counting function $v_{(\Gamma, S)}$ as

$$
v_{(\Gamma, S)}(k):=\# B_{S}(k) .
$$

A finitely generated group $\Gamma$ has polynomial growth if for one (hence any) generating set $S$, there exists a polynomial $P_{S}$ such that

$$
v_{(\Gamma, S)}(k) \leq P_{S}(k) .
$$

A finitely generated group $\Gamma$ has exponential growth if for one (hence any) generating set $S$, there exists $C_{S}>0$ such that

$$
v_{(\Gamma, S)}(k) \geq e^{C_{S} k} .
$$

Examples 5.1. 1) For $\Gamma=\mathbf{Z}^{n}$ and for every generating set $S$, we have

$$
v_{(\Gamma, S)}(k) \approx k^{n} .
$$

2) For the non abelian free group $\Gamma=\mathbf{F}_{n}$ on $n$ generators, $n \geq 2$ and for every generating set $S$, we have

$$
v_{(\Gamma, S)}(k) \approx(2 n-1)^{k} .
$$

3) Let $\Gamma:=\operatorname{Heis}(\mathbf{Z})$ where

$$
\operatorname{Heis}(\mathbf{Z})=\left\{\left(\begin{array}{ccc}
1 & x & z \\
0 & 1 & y \\
0 & 0 & 1
\end{array}\right) / \mathrm{x}, \mathrm{y}, \mathrm{z} \in \mathbf{Z}\right\} .
$$

Then, for every generating set $S$, we have

$$
v_{(\Gamma, S)}(k) \approx k^{4} .
$$

4) More generally, let $\Gamma$ be a finitely generated nilpotent group, and $\{1\} \unlhd \Gamma_{n} \unlhd \cdots \unlhd$ $\Gamma_{1}=\Gamma$ its lower central series, where $\left[\Gamma_{k}, \Gamma\right]=\Gamma_{k+1}$. Then, for every generating set of $\Gamma$, the growth of $\Gamma$ is given by the formula due to Bass-Guivarc' $h$,

$$
v_{(\Gamma, S)}(k) \approx k^{d(\Gamma)}
$$

where $d(\Gamma)=\sum_{k} k \operatorname{rank}\left(\Gamma_{\mathrm{k}} / \Gamma_{\mathrm{k}+1}\right)$. In particular, every finitely generated nilpotent group has polynomial growth, (see [Gui71], [Bas72]). 
We now have the following easy lemma, saying that a group $(\Gamma, S)$ with polynomial growth is " $C$-doubling" at scale $k_{n}$ for a sequence $k_{n}$ tending to infinity, i.e. $\frac{v_{(\Gamma, S)}\left(2 k_{n}\right)}{v_{(\Gamma, S)}\left(k_{n}\right)} \leq$ $C$, see the regularity Lemma p. 59 in [Gro81] for a more precise statement.

Lemma 5.2. Let us consider a finitely generated group $\Gamma$ with polynomial growth. Let $\mu$ be the counting measure on the Cayley graph $\mathcal{G}(\Gamma, S)$. We claim that there exist $C>1$ and a sequence of integers $k_{n}$ tending to infinity such that

$$
\frac{\mu\left(B_{S}\left(2 k_{n}\right)\right)}{\mu\left(B_{S}\left(k_{n}\right)\right)}=\frac{v_{(\Gamma, S)}\left(2 k_{n}\right)}{v_{(\Gamma, S)}\left(k_{n}\right)} \leq C .
$$

Proof. Let us prove this assertion. We assume by contradiction that for every $C>1$, there exists $N \in \mathbf{N}$ such that, for every $n \geq N$, we have

$$
\frac{v_{(\Gamma, S)}(2 n)}{v_{(\Gamma, S)}(n)} \geq C .
$$

In particular, for every integer $k$, we have $v_{(\Gamma, S)}\left(2^{k} n\right) \geq v_{(\Gamma, S)}(n) C^{k}$. Therefore, for every $x \geq n$, we get

$$
v_{(\Gamma, S)}(x) \geq \frac{v_{(\Gamma, S)}(n)}{n^{\frac{\log C}{\log 2}}} x^{\frac{\log C}{\log 2}},
$$

which contradicts the polynomial growth of $\Gamma$ since $C$ is arbitrary.

Examples 5.3. 1) If $\Gamma=\mathbf{Z}^{n}$ and $S$ its canonical generating set, then,

$$
\frac{v_{(\Gamma, S)}(2 k)}{v_{(\Gamma, S)}(k)}=2^{n} .
$$

2) For $\Gamma$ nilpotent, it follows directly from Bass-Guivarc'h estimate of the growth that

$$
\frac{v_{(\Gamma, S)}(2 k)}{v_{(\Gamma, S)}(k)} \approx 2^{d(\Gamma)} .
$$

3) If $\Gamma=\mathbf{F}_{n}$ is the free group on $n$ generators and $S$ the symmetric set of generators, then

$$
\frac{v_{(\Gamma, S)}(2 k)}{v_{(\Gamma, S)}(k)} \approx(2 n-1)^{k} .
$$

For a finitely generated group $\Gamma$, having polynomial growth is a strong constraint. The following theorem, due to Gromov, characterises the groups with polynomial growth.

Theorem 5.4 ([Gro81]). Let $\Gamma$ be a finite generated group with polynomial growth. Then, $\Gamma$ is virtually nilpotent.

According to the lemma 5.2, every group with polynomial growth is $C$-doubling at scale $k_{n}$, for some $C>1$ and some sequence $k_{n}$ tending to infinity. This property of groups with polynomial growth is a step in the proof of Theorem 5.4. With the following observation in mind, the next theorem, due to Breuillard, Green and Tao may be considered as a strong version of Gromov's Theorem. It says roughly that if a group is $p$-doubling at one sufficiently large scale with respect to $p$, then $G$ is virtually nilpotent. 
Theorem 5.5 ([BGT12]). For every $p \in \mathbf{N} \backslash\{0\}$, there exists $N(p) \in \mathbf{N} \backslash\{0\}$ such that the following holds: for every group $G$ and every generating set $S$ of $G$, if there exists a finite subset $A \subset G$ such that

1. $S^{N(p)} \subset A$

2. $\frac{\# A \cdot A}{\# A} \leq p$

then, $G$ is virtually nilpotent.

Remark 5.6. In the previous theorem, the hypothesis 2 is a p-doubling condition for the set $A$, while the first one means that the doubling holds at one given scale larger than a universal function $N(p)$ of $p$.

\subsection{A Margulis Lemma}

Let $\Gamma$ be a group acting properly discontinuously on a metric space $(X, d)$. For every $x \in X$ and $r>0$, we set

$$
\Gamma_{r}(x):=\{\gamma \in \Gamma \mid d(x, \gamma x) \leq r\} .
$$

When $X$ is simply-connected then $\Gamma$ is the fundamental group of $X / \Gamma$ and $\Gamma_{r}(x)$ is nothing but the subgroup of $\Gamma$ generated by the "small loops" at $p$ of length less than $r$, where $p$ is the point on $X / \Gamma$ having $x$ as a lift. We will denote this subgroup $\Gamma_{r}(p)$ as well. The following fundamental Theorem due to Margulis says that such groups generated by "small loops" at a point are virtually nilpotent when $r$ is small enough, which gives strong informations on the geometry of neigbourhoods of such points.

Theorem 5.7 ([Mar75], [BGS85], [BZ88] Section 37.3). There exists a positive constant $\varepsilon(n, a)$ such that, for every complete Riemannian manifold $M$ whose sectional curvature satisfies $-a^{2} \leq \operatorname{Sect}(M) \leq 0$, for every point $p \in M$ and for every $\varepsilon \leq \varepsilon(n, a)$, the subgroup $\Gamma_{\varepsilon}(p)$ of $\pi_{1}(M)$ generated by the loops at $p$ of length less than $\varepsilon$ is virtually nilpotent.

We emphasise that the constant $\varepsilon(n, a)$ depends only on the curvature bound and the dimension. This theorem has been extended to various settings, we do not intend to survey here all of these and refer to [KW11], [BGT12], [Cou15], [BCGS20b]. In particular, in [BGT12], the authors deduce from Theorem 5.5 a "Ricci gap Theorem", saying that if an $n$-dimensional Riemannian manifold $\left(M^{n}, g\right)$ with $\operatorname{diam} M \leq 1$ satisfies $\operatorname{Ric}_{g} \geq \epsilon(n)$, then the fundamental group of $M^{n}$ is virtually nilpotent. The purpose of this lecture is to describe how our Bishop-Gromov Theorem 1.8 allows to prove a Margulis Lemma in the context of groups acting on metric spaces where the negative curvature assumption is replaced by $\delta$-hyperbolicity and the lower curvature bound by an upper bound on the entropy .

Remark 5.8. The following observation is classical, we leave it as an exercise. Let $\Gamma$ acting properly discontinuously by isometries on a Gromov hyperbolic space $(X, d)$ be such that $X / \Gamma$ is compact. Then, every virtually nilpotent subgroup of $\Gamma$ is virtually cyclic (see also [BCGS20b], Section 8). 
Theorem 5.5 provides a sufficient condition for a group $\Gamma$ to be (virtually) nilpotent. This condition is formulated by the $p$-doubling property of the Cayley graph of $\Gamma$ at a scale given by the universal function $N(p)$. On the other hand, for a group $\Gamma$ acting on a $\delta$-hyperbolic space $X$ with entropy bounded by $H$ and diameter of the quotient space $X / \Gamma$ bounded by $D$, our Bishop-Gromov Theorem 1.8 says that $\Gamma$ is $p$-doubling at scale $R_{0}$ for $p:=p(\delta, D, H)$ and $R_{0}:=R_{0}(\delta, D)$. Theorem 5.5 then allows to translate the universal function

$$
\epsilon(\delta, H, D):=\frac{R_{0}}{N(p)}
$$

into a Margulis constant for such actions.

Theorem 5.9 ([BCGS20a]). For every $\delta \geq 0, H \geq 0$, and $D \geq 0$, there exists $\epsilon_{0}:=$ $\epsilon_{0}(\delta, H, D)$ such that the following holds. Let $(X, d)$ be a $\delta$-hyperbolic space, and $\Gamma$ acting isometrically and properly on $X$. Assume that $\operatorname{diam} X / \Gamma \leq D$ and $\operatorname{Ent}(\mathrm{X}, \mathrm{d}) \leq \mathrm{H}$. Then, for every $r \leq \epsilon_{0}$ and every $x \in X$, the group $\Gamma_{r}(x)$ is virtually cyclic.

Proof. Let $N_{0}:=N\left(3^{10} e^{300 H(D+2 \delta)}+1\right)$ and $R_{0}:=20(D+2 \delta)$, where $N$ is the function defined in Theorem 5.5. Denote by $\epsilon_{0}$ the constant $\frac{R_{0}}{N_{0}}$ and choose $0<r \leq \epsilon_{0}$.

We consider $G:=\Gamma_{r}(x)$ the subgroup of $\Gamma$ generated by $S:=\{g \in \Gamma \mid d(x, g x) \leq r\}$, and $A \subset G$ where $A=\left\{g \in G \mid d(x, g x) \leq R_{0}\right\}$. We will show that $G$ and $A$ satisfy the hypotheses of Theorem 5.5, which will imply that $G=\Gamma_{r}(x)$ is virtually nilpotent, hence cyclic since $X$ is $\delta$-hyperbolic.

Let us check the hypothesis 2 . Let us denote $\mu_{x}^{G}=\sum_{g \in G} \delta_{g x}$ the counting measure of the $G$-orbit of $x$ in $X$. Notice that $A \cdot A \subset\left\{g \in G \mid d(x, g x) \leq 2 R_{0}\right\}$, hence

$$
\frac{\# A \cdot A}{\# A} \leq \frac{\mu_{x}^{G}\left(B\left(x, 2 R_{0}\right)\right)}{\mu_{x}^{G}\left(B\left(x, R_{0}\right)\right)} .
$$

By the Bishop-Gromov inequality for subgroup $G \leq \Gamma$, cf. Corollary 4.9, we have

$$
\frac{\mu_{x}^{G}\left(B\left(x, 2 R_{0}\right)\right)}{\mu_{x}^{G}\left(B\left(x, R_{0}\right)\right)} \leq \frac{\mu_{x}^{\Gamma}\left(B\left(x, 2 R_{0}+R_{0} / 2\right)\right)}{\mu_{x}^{\Gamma}\left(B\left(x, R_{0} / 2\right)\right)}
$$

Applying the Bishop-Gromov theorem 1.8 for $\Gamma$ and for $(r, R)$ replaced by $\left(\frac{R_{0}}{2}, \frac{5}{2} R_{0}\right)$, we get

$$
\frac{\mu_{x}^{\Gamma}\left(B\left(x, 2 R_{0}+R_{0} / 2\right)\right)}{\mu_{x}^{\Gamma}\left(B\left(x, R_{0} / 2\right)\right)} \leq 3 \cdot 5^{25 / 4} e^{15 H R_{0}} \leq 3^{10} e^{300 H(D+2 \delta)} .
$$

By the three previous inequalities, we deduce the hypothesis 2 of Theorem 5.5

$$
\frac{\# A \cdot A}{\# A} \leq p
$$

with $p:=3^{10} e^{300 H(D+2 \delta)}$.

We now check the hypothesis 1 . Recall that we have chosen

$$
N_{0}:=N(p+1)=N\left(3^{10} e^{300 H(D+2 \delta)}+1\right),
$$


$R_{0}=20(D+2 \delta)$ and $0<r \leq \epsilon_{0}=\frac{R_{0}}{N_{0}}$.

By definition of the generating set $S$, for every $g \in S$, we have $d(x, g x) \leq r$. Every $g \in S^{N_{0}}$ can be written as $g=g_{1} g_{2} \cdots g_{k}$ with $k \leq N_{0}$, hence by the triangle inequality,

$$
d(x, g x) \leq k r \leq N_{0} r \leq R_{0},
$$

and therefore, $g \in A$, which proves that $S^{N_{0}} \subset A$ and the hypothesis 1 .

By Theorem 5.5, we therefore deduce that $\Gamma_{r}(x)$ is virtually nilpotent. We now notice that, since $(X, d)$ is Gromov hyperbolic and $X / \Gamma$ is compact, the virtually nilpotent subgroups of $\Gamma$ are actually virtually cyclic by Remark 5.8, which ends the proof of Theorem 5.9.

\subsection{The thin-thick decomposition}

This section gives geometric applications of Theorem 5.9, These applications are classical in the case of manifolds with bounded non positive curvature. Let $\Gamma$ a group acting freely and properly discontinuously on $(X, d)$. In this section, we will assume $X$ non compact and $\Gamma$ torsion free. The systole of this action at $x \in X$ is defined as

Definition 5.10. $\operatorname{sys}_{\Gamma}(x):=\inf _{\gamma \in \Gamma \backslash\{I d\}} d(x, \gamma x)$.

For every $r>0$, the $r$-thin part of the action of $\Gamma$ is the open set defined as

Definition 5.11. $X_{r}:=\left\{x \in X \mid \operatorname{sys}_{\Gamma}(x)<r\right\}$.

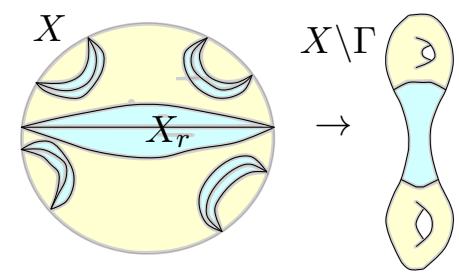

Figure 5.1: r-Thin part

The $r$-thick part is simply defined as $X \backslash X_{r}$. Notice that the thin and thick parts are defined as subsets of $X$. When $(X, d)$ is Gromov hyperbolic, the action of $\Gamma$ on $(X, d)$ non elementary and $X / \Gamma$ compact, then the thin part is not connected, as shown in the next proposition. We recall that $\Gamma$ is assumed to be torsion free.

Proposition 5.12. Let $\Gamma$ acting properly discontinuously on a Gromov hyperbolic space $(X, d)$ such that $X / \Gamma$ is compact, and let $0<r \leq \epsilon_{0}$, where $\epsilon_{0}:=\epsilon_{0}(\delta, H, D)$ is the constant of Theorem 5.9. We assume that the action of $\Gamma$ is non elementary. Then,

1. For every $x \in X_{r}$, there exists a maximal virtually cyclic subgroup $G(x) \subset \Gamma$ containing $\Sigma_{r}(x):=\{\gamma \in \Gamma \mid d(x, \gamma x) \leq r\}$. The map $x \rightarrow G(x)$, defined on $X_{r}$, is locally constant, hence constant on each connected component of $X_{r}$. 
2. If $X_{r} \neq \emptyset$, then $X_{r}$ is not connected.

Proof. Let us prove the first part of the proposition. Let us consider $0<r \leq \epsilon_{0}$ and $\gamma \in \Sigma_{r}(x)$ for some $x \in X_{r}$. Let us assume, to simplify, that $\gamma$ is a non trivial hyperbolic isometry with fixed points $\gamma_{ \pm}$on the boundary of $X$ (for these notions the reader is referred to [BCGS20b], Section 8). Notice that every element of $\Gamma_{r}(x)$ fixes the set $\left\{\gamma^{-}, \gamma^{+}\right\}$

The group

$$
G(x):=\left\{g \in \Gamma \mid g\left(\left\{\gamma_{-}, \gamma_{+}\right\}\right)=\left\{\gamma_{-}, \gamma_{+}\right\}\right\},
$$

is the unique maximal cyclic subgroup of $\Gamma$ containing $\gamma$. We then have

$$
\Sigma_{r}(x) \subset \Gamma_{r}(x) \leq G(x) .
$$

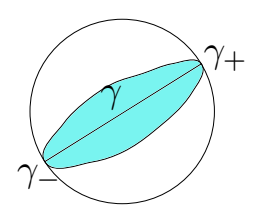

Figure 5.2: Hyperbolic isometry

Let us show that the map defined on $X_{r}$ by $x \rightarrow G(x)$ is locally constant. Since the action of $\Gamma$ is proper, there exists $\beta:=\beta(x)>0$ be such that

$$
\min _{\gamma \in \Gamma \backslash \Sigma_{r}(x)} d(x, \gamma x)>r+\beta .
$$

Let us consider $x^{\prime}$ such that $d\left(x, x^{\prime}\right) \leq \beta / 2$. We claim that $\Sigma_{r}\left(x^{\prime}\right) \subset \Sigma_{r}(x)$. Indeed, take $\gamma \in \Sigma_{r}\left(x^{\prime}\right)$, we then have

$$
d(x, \gamma x) \leq d\left(x, x^{\prime}\right)+d\left(x^{\prime}, \gamma x^{\prime}\right)+d\left(\gamma x^{\prime}, \gamma x\right) \leq r+\beta .
$$

Therefore, $G\left(x^{\prime}\right) \leq G(x)$ hence $G\left(x^{\prime}\right)=G(x)$ by maximality. This shows that the map $x \rightarrow G(x)$ is locally constant on $X_{r}$ and concludes the part 1 .

We now prove the part 2 of the proposition. Let us assume, by contradiction, that $X_{r}$ is non empty and connected. Then, since $G(x)$ is locally constant, $G(x)=G$ does not depend on $x \in X_{r}$. Notice that $X_{r}$ is $\Gamma$-invariant, $g X_{r}=X_{r}$ for every $g \in \Gamma$, and $\Sigma_{r}(g x)=g \Sigma_{r}(x) g^{-1}$ for every $x \in X_{r}$ and $g \in \Gamma$. Given $x \in X$, recall that $G(x):=\left\{g \in \Gamma \mid g\left(\left\{\gamma_{-}, \gamma_{+}\right\}\right)=\left\{\gamma_{-}, \gamma_{+}\right\}\right\}$, where $\left\{\gamma_{-}, \gamma_{+}\right\}$are the fixed points of some hyperbolic isometry $\gamma \in \Sigma_{r}(x)$. Hence for every $g \in \Gamma, g \gamma g^{-1} \in \Sigma_{r}(g x) \subset G(g x)=$ $G=G(x)$. In particular, since the set of fixed points of $g \gamma g^{-1}$ is $\left\{g \gamma_{-}, g \gamma_{+}\right\}$, we deduce that $\left\{g \gamma_{-}, g \gamma_{+}\right\}=\left\{\gamma_{-}, \gamma_{+}\right\}$for every $g \in \Gamma$, hence $\Gamma=G(x)$ and $\Gamma$ is elementary, a contradiction. 


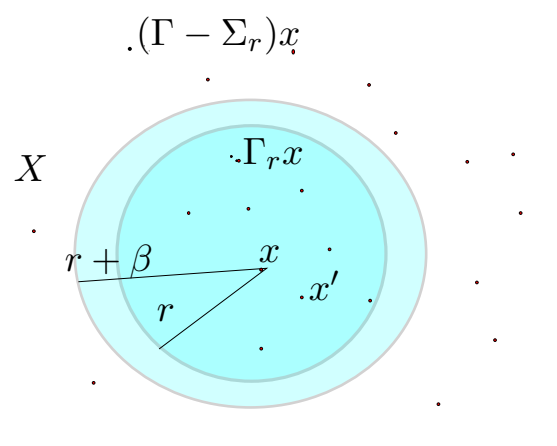

Figure 5.3: $G(x)$ locally constant

In the following proposition, we show that if a group $\Gamma$ acts properly on a $\delta$-hyperbolic space $(X, d)$ with bounded entropy and bounded co-diameter, then there exists a point on $X$ with a large systole.

Proposition 5.13. Let $\Gamma$ act properly discontinuously on a $\delta$-Gromov hyperbolic space $(X, d)$ such that $\operatorname{diam}(X / \Gamma) \leq D$ and $\operatorname{Ent}(\mathrm{X}, \mathrm{d}) \leq \mathrm{H}$ and let $\epsilon_{0}:=\epsilon_{0}(\delta, H, D)$ be the constant of Theorem 5.9. We assume that the action of $\Gamma$ is non elementary and that $\Gamma$ is torsion free. Then, there exists a point $x \in X$ such that $\operatorname{sys}_{\Gamma}(x) \geq \epsilon_{0}$.

Proof. By Theorem 5.9, there exists $\epsilon_{0}:=\epsilon_{0}(\delta, H, D)>0$ such that for every $0<r \leq \epsilon_{0}$ and every $x \in X, \Gamma_{r}(x)$ is cyclic. Now there are two cases. If $X_{\epsilon_{0}}=\emptyset$, there is nothing to prove. If $X_{\epsilon_{0}} \neq \emptyset$, then $X_{r}$ is not connected and since $X$ is connected, we deduce that $X \backslash X_{r} \neq \emptyset$ and there exists $x \in X$ such that $\operatorname{sys}_{\Gamma}(x) \geq \epsilon_{0}$.

\section{Lecture 6}

In this lecture we recall the definition of the systole of a proper and geodesic metric space $(X, d)$ on which a discrete subgroup $\Gamma$ of its isometry group acts. For the sake of simplicity we set $\Gamma^{*}:=\Gamma \backslash\{e\}$, where $e$ is the neutral element of $\Gamma$. For the next results we need to exclude "trivial" hyperbolic spaces which we call elementary, we recall below the definition.

Definition 6.1. A hyperbolic space or group is called elementary if its ideal boundary has at most two points.

\subsection{Systoles}

We recall the definition of the systole at a point given in Lecture 5 (see Definition 5.10) and we define the global systole of a group action.

Definition 6.2. Let $(X, d)$ be as above, we define 
i) $\forall x \in X$, the systole at $x$ is

$$
\operatorname{sys}_{\Gamma}(x)=\inf _{\gamma \in \Gamma^{*}}\{d(x, \gamma x)\} .
$$

ii) The global systole of the action of $\Gamma$ is

$$
\operatorname{Sys}_{\Gamma}(X)=\inf _{x \in X}\left\{\operatorname{sys}_{\Gamma}(x)\right\} .
$$

Remark 6.3. If $x_{0} \in X$ is fixed by some element $\gamma_{0} \in \Gamma$, i.e. if $d\left(x_{0}, \gamma_{0} x_{0}\right)=0$, then

$$
\operatorname{Sys}_{\Gamma}(X)=\operatorname{sys}_{\Gamma}\left(x_{0}\right)=0 .
$$

We may say that $\gamma_{0} \in \operatorname{Isom}(X, d)$ is an elliptic element. In the sequel we shall always assume that the space $(X, d)$ is $\delta$-hyperbolic for some $\delta \geq 0$ in which case an element $\gamma \in \Gamma$ is elliptic if and only if it is a torsion element (see Remark 8.16 in [BCGS20b]). For the sake of simplicity we shall assume in the sequel that all isometries in $\Gamma$ are torsion-free. For the general case the reader is refered to [BCGS20b].

\subsection{Bounding from below the Systole}

We now intend to prove the following theorem.

Theorem 6.4. Let $\delta \geq 0$ and $H, D>0$. There exists $s_{0}:=s_{0}(\delta, H, D)>0$ with the following property. Let $(X, d)$ be a non-elementary $\delta$-hyperbolic space which is Busemann and satisfies the property of extension of the geodesics. If $\Gamma \subset \operatorname{Isom}(X, d)$ is discrete, torsion-free and such that,

$$
\operatorname{diam}(X / \Gamma, \bar{d}) \leq D \quad \text { and } \quad \operatorname{Ent}(X, d) \leq H,
$$

then,

$$
\forall x \in X, \quad \forall \gamma \in \Gamma^{*}, \quad d(x, \gamma x)>s_{0}(\delta, H, D) .
$$

Then,

$$
\operatorname{Sys}_{\Gamma}(X)>s_{0}(\delta, H, D) .
$$

To understand this result and its proof one should look at the simplest case, namely the collar lemma for hyperbolic (constant curvature) surfaces summarised in the picture below as well as the separation between thin and thick parts.

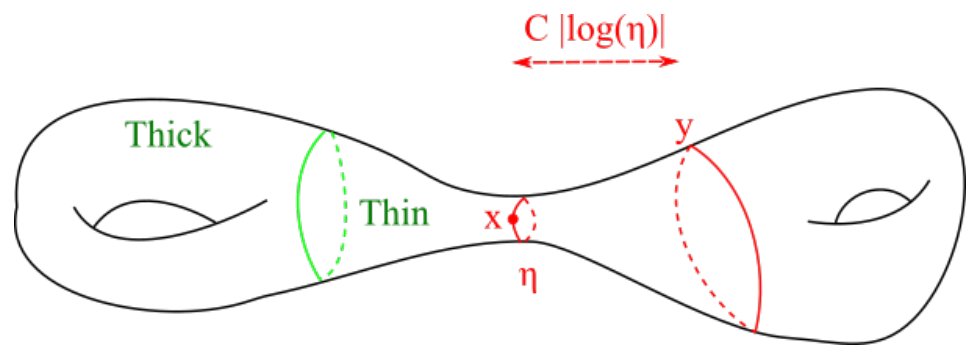

Figure 6.1: Classical Collar Lemma 
Here we have $\operatorname{sys}_{\Gamma}(x)=\eta \ll 1$, $\operatorname{sys}_{\Gamma}(y) \geq \epsilon_{\mathrm{Mar}}$, where $\epsilon_{\text {Mar }}$ denotes the Margulis constant of the surface, and the collar lemma asserts that,

$$
d(x, y) \geq C|\log (\eta)| .
$$

Proof of Theorem 6.4. For $\gamma \in \Gamma^{*}$ we define,

$$
G=<\gamma>=\left\{\gamma^{k} ; k \in \mathbf{Z}\right\},
$$

the cyclic group generated by $\gamma$.

Lemma 6.5 (Collar Lemma). Let $\Gamma$ act properly on $(X, d)$ and let $R>0, \gamma \in \Gamma^{*}$ and $x \in X$. If there exists $y_{0} \in X$ and $\epsilon>0$ such that,

$$
\inf _{k \in N^{*}}\left\{d\left(y_{0}, \gamma^{k} y_{0}\right)\right\}>\epsilon>0 \quad \text { and } \quad d\left(x, y_{0}\right) \leq R
$$

then,

$$
\frac{d(x, \gamma x)}{4 R} \geq\left(\frac{\mu_{y_{0}}^{\Gamma}\left(B\left(y_{0}, 4 R+\epsilon / 2\right)\right)}{\mu_{y_{0}}^{\Gamma}\left(B\left(y_{0}, \epsilon / 2\right)\right)}+1\right)^{-1} .
$$

This is our version of the classical Collar lemma. Notice that the denominator of the left hand side never vanishes. Indeed we always have $\mu_{y_{0}}^{\Gamma}\left(B\left(y_{0}, \epsilon / 2\right)\right) \geq 1$ since $y_{0}$ is a point of its own orbit by $\Gamma$ in $B\left(y_{0}, \epsilon / 2\right)$.

Proof of Lemma 6.5. By the triangle inequality one has,

$$
d\left(x, \gamma^{k} x\right) \leq d(x, \gamma x)+d\left(\gamma x, \gamma^{2} x\right)+\cdots+d\left(\gamma^{k-1} x, \gamma^{k} x\right) \leq k d(x, \gamma x),
$$

so that, if $|k| \leq\left[\frac{2 R}{d(x, \gamma x)}\right]$ then, $d\left(x, \gamma^{k} x\right) \leq 2 R$. We then obtain,

$$
2\left[\frac{2 R}{d(x, \gamma x)}\right]+1 \leq \#\left\{k ; d\left(x, \gamma^{k} x\right) \leq 2 R\right\}=\mu_{x}^{G}(\bar{B}(x, 2 R)) .
$$

Now,

$$
d\left(y_{0}, \gamma^{k} y_{0}\right) \leq d\left(y_{0}, x\right)+d\left(x, \gamma^{k} x\right)+d\left(\gamma^{k} x, \gamma^{k} y_{0}\right) \leq 2 d\left(y_{0}, x\right)+d\left(x, \gamma^{k} x\right),
$$

which implies that if $d\left(x, \gamma^{k} x\right) \leq 2 R$ then $d\left(y_{0}, \gamma^{k} y_{0}\right) \leq 4 R$.

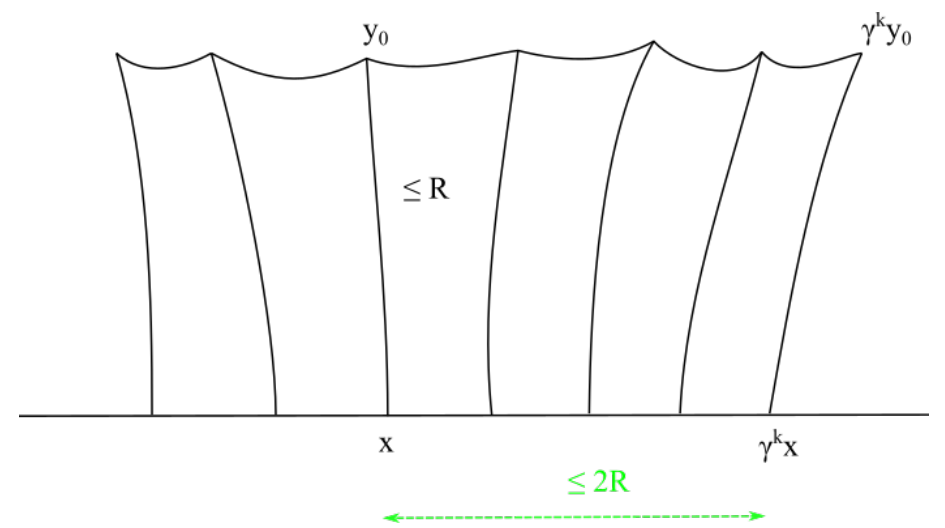

Figure 6.2: Proof of the Collar lemma 
This leads to

$$
\mu_{x}^{G}(\bar{B}(x, 2 R)) \leq \mu_{y_{0}}^{G}\left(\bar{B}\left(y_{0}, 4 R\right)\right) .
$$

These inequalities lead to

$$
\frac{4 R}{d(x, \gamma x)}-1 \leq 2\left[\frac{2 R}{d(x, \gamma x)}\right]+1 \leq \frac{\mu_{y_{0}}^{G}\left(\bar{B}\left(y_{0}, 4 R\right)\right)}{\mu_{y_{0}}^{G}\left(\bar{B}\left(y_{0}, \epsilon\right)\right)} \leq \frac{\mu_{y_{0}}^{\Gamma}\left(B\left(y_{0}, 4 R+\epsilon / 2\right)\right)}{\mu_{y_{0}}^{\Gamma}\left(B\left(y_{0}, \epsilon / 2\right)\right)}
$$

The second inequality comes from the fact that $\mu_{y_{0}}^{G}\left(\bar{B}\left(y_{0}, \epsilon\right)\right)=1$ since, by definition of $\epsilon, y_{0}$ is the only point of its orbit by $G$ in $\bar{B}\left(y_{0}, \epsilon\right)$. The third inequality comes from Corollary 4.9 applied with $\Gamma^{\prime}=G$.

We come back to the proof of Theorem 6.4. We pick $x \in X$, and $\gamma \in \Gamma^{*}$. From Proposition 5.13 there exists $x_{0} \in X$ such that $\forall \alpha \in \Gamma^{*}$,

$$
d\left(x_{0}, \alpha x_{0}\right) \geq \epsilon_{0}=\epsilon_{0}(\delta, H, D),
$$

where $\epsilon_{0}(\delta, H, D)$ is the constant introduced in Theorem 5.9. In particular, $\forall k \neq 0$, $d\left(x_{0}, \gamma^{k} x_{0}\right) \geq \epsilon_{0}>0$. However, $x_{0}$ may not be close to $x$ as required by Lemma 6.5. Now, because of the fact that the action of $\Gamma$ is co-compact and that the quotient has diameter bounded above by $D$ there exists $\alpha_{0} \in \Gamma$ such that $d\left(x, \alpha_{0} x_{0}\right) \leq D$. We set $y_{0}=\alpha_{0} x_{0}$ and $\forall k \neq 0$,

$$
d\left(y_{0}, \gamma^{k} y_{0}\right)=d\left(\alpha_{0} x_{0}, \gamma^{k} \alpha_{0} x_{0}\right)=d\left(x_{0}, \alpha_{0}^{-1} \gamma^{k} \alpha_{0} x_{0}\right) \geq \epsilon_{0}>0,
$$

since $\alpha_{0}^{-1} \gamma^{k} \alpha_{0} \in \Gamma$. We then apply the previous lemma with $R=D$ and $\epsilon_{0}$ instead of $\epsilon$ and we get,

$$
d(x, \gamma x) \geq 4 D\left(\frac{\mu_{y_{0}}^{\Gamma}\left(B\left(y_{0}, 4 D+\epsilon_{0} / 2\right)\right)}{\mu_{y_{0}}^{\Gamma}\left(B\left(y_{0}, \epsilon_{0} / 2\right)\right)}+1\right)^{-1} .
$$

Then it suffices to apply our Bishop-Gromov Inequality to get a lower bound for $d(x, \gamma x)$, for any $x \in X$ and any $\gamma \in \Gamma^{*}$, in term of $\delta, H$ and $D$ and also $\epsilon_{0}$ which itself depends on $\delta, H$ and $D$. The resulting function of $\delta, H$ and $D$ is called $s_{0}$. Notice that this is unfortunately not explicit since $\epsilon_{0}(\delta, H, D)$ is not. This ends the proof of Theorem 6.4

\section{Lecture 7}

In this lecture, we study finiteness properties of isomorphism classes of finitely generated groups acting cocompactly on a $\delta$-hyperbolic metric space with bounded entropy. To be more precise, we consider the class of metric spaces $(X, d)$ which satisfy the following assumptions: $(X, d)$ is complete, non elementary $\delta$-hyperbolic and Busemann. The main statement is that the set of isomorphism classes of group $\Gamma$ which have a proper isometric action on such a space $(X, d)$ with $\operatorname{diam}(X / \Gamma, d) \leq D$ and $\operatorname{Ent}(X, d) \leq H$ has less than $M_{0}(\delta, H, D)$ elements. There are two steps in the proof. One is to find a generating 
set $S$ of $\Gamma$ with bounded cardinality $\# S \leq n_{0}(\delta, H, D)$. This is a consequence of our Bishop-Gromov Theorem 4.3 and the diastole estimate, cf. Lecture 5, Proposition 5.13. The second step consists in bounding the number of presentations of the groups $\Gamma$. This is a consequence of the fact that $\Gamma$ inherits the Gromov hyperbolicity of $(X, d)$ and that hyperbolic groups admit bounded presentations.

\subsection{Marked groups}

In this section, we introduce the notion of "marked groups" which will be useful for formulating the finiteness of isomorphism classes of groups. Given a finitely generated group $(\Gamma, S)$, where $S$ denotes a symmetric set of generators $S:=\left\{\sigma_{1}, \cdots, \sigma_{k}, \sigma_{1}^{-1}, \cdots, \sigma_{k}^{-1}\right\}$, and the non abelian free group on $k$ generators $\mathbf{F}_{k}:=\left(\mathbf{F}_{k},\left\{e_{1}, \cdots, e_{k}, e_{1}^{-1}, \cdots, e_{k}^{-1}\right\}\right)$, we have a morphism

$$
\varphi_{\Gamma, S}: \mathbf{F}_{k} \rightarrow \Gamma
$$

defined by $\varphi_{\Gamma, S}\left(e_{i}^{ \pm 1}\right)=\sigma_{i}^{ \pm 1}$. In particular, we have

$$
\Gamma \simeq \mathbf{F}_{k} / \operatorname{Ker} \varphi_{\Gamma, \mathrm{S}} .
$$

Definition 7.1. A marked group is the data of a finitely generated group $(\Gamma, S)$ with the morphism $\varphi_{\Gamma, S}$. We call $\varphi_{\Gamma, S}$ the marking.

As we are interested in isomorphism classes of groups, we define:

Definition 7.2. An isomorphism $\beta:(\Gamma, S) \rightarrow\left(\Gamma^{\prime}, S^{\prime}\right)$ is an isomorphism $\beta: \Gamma \rightarrow \Gamma^{\prime}$ such that $\beta\left(\sigma_{i}\right)=\sigma_{i}^{\prime}$. In particular, $\varphi_{\Gamma^{\prime}, S^{\prime}}=\beta \circ \varphi_{\Gamma, S}$ and we shall call such a $\beta$ an isomorphism of marked groups, denoted by $(\Gamma, S) \simeq\left(\Gamma^{\prime}, S^{\prime}\right)$.

Remark 7.3. Notice that an isomorphism $\beta:(\Gamma, S) \rightarrow\left(\Gamma^{\prime}, S^{\prime}\right)$ induces an isometry between the Cayley graphs:

$$
\bar{\beta}:\left(\mathcal{G}(\Gamma, S), d_{S}\right) \rightarrow\left(\mathcal{G}\left(\Gamma^{\prime}, S^{\prime}\right), d_{S^{\prime}}\right) .
$$

The next classical Proposition states that, when $\Gamma$ acts by isometries properly discontinuously and cocompactly on $(X, d)$, then $\Gamma$ is finitely generated and the Gromov hyperbolicity transfers from the space $(X, d)$ to the Cayley graph $\left(\mathcal{G}(\Gamma, S), d_{S}\right)$ for every set $S$ of generators. The main idea is that, under the cocompactness assumption, the Cayley graph of $\Gamma$ is quasiisometric to $(X, d)$. Gromov hyperbolicity is then a property which is invariant by quasiisometry, [CDP90], [BH99].

Proposition 7.4. Let $(X, d)$ be a $\delta$-hyperbolic metric space and $\Gamma$ acting properly discontinuously by isometries on $(X, d)$. Assume that $\operatorname{diam}(X / \Gamma) \leq D$. Let $x \in X$, then $S:=S_{6 D}=\{\gamma \in \Gamma \mid d(x, \gamma x) \leq 6 D\}$ is a generating set of $\Gamma$ and $(\Gamma, S)$ is $\delta^{\prime}$-hyperbolic for $\delta^{\prime}:=8\left(\frac{5 \delta}{D}+4\right)$.

Proof. We only sketch the proof; for details we refer to [BCGS20a]. Let us choose a point $x \in X$. We first observe that for every $k \in \mathbf{N}^{*}$, the set

$$
S:=S_{(k+2) D}(x)=\{\gamma \in \Gamma \mid d(x, \gamma x) \leq(k+2) D\}
$$


is a generating set of $\Gamma$. For $\gamma \in \Gamma$, set $n:=\left\lfloor\frac{d(x, \gamma x)}{k D}\right\rfloor+1$. On a geodesic $[x, \gamma x]$, let us choose $x_{0}=x, x_{1}, \cdots, x_{n}=\gamma x$ such that $d\left(x_{i-1}, x_{i}\right)=k D$ for $1 \leq i \leq n-1$, and $d\left(x_{n-1}, x_{n}\right)<k D$. By the assumption on the diameter, for every $1 \leq i \leq n$, there is $\gamma_{i} \in \Gamma$ such that $d\left(x_{i}, \gamma_{i} x\right) \leq D$. Then, defining $\gamma_{0}=I d_{X}, \gamma_{n}:=\gamma$ and $\sigma_{i}:=\gamma_{i-1}^{-1} \gamma_{i}$ for $i=1, \cdots, n$, we see that $\gamma=\sigma_{1} \cdots \sigma_{n}$, and moreover,

$$
d\left(x, \sigma_{i} x\right) \leq d\left(\gamma_{i-1} x, x_{i-1}\right)+d\left(x_{i-1}, x_{i}\right)+d\left(x_{i}, \gamma_{i} x\right) \leq(k+2) D .
$$

This implies that $S_{(k+2) D}:=\{\sigma \in \Gamma \mid d(x, \sigma x) \leq(k+2) D\}$ is a generating set of $\Gamma$. Choosing $k=4$, we see that $S:=S_{6 D}$ is a generating set of $\Gamma$, which proves the first part of the proposition.

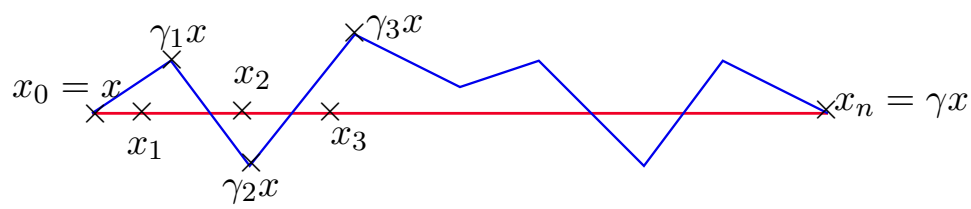

Figure 7.1: $S_{(k+2) D}$

From now on, we fix $S=S_{6 D}$. In order to prove that $(\Gamma, S)$ is $\delta^{\prime}$-hyperbolic, we first show that the Cayley graph of $(\Gamma, S)$ is "quasiisometric" to $(X, d)$ and then, deduce that $(\Gamma, S)$ is $\delta^{\prime}$-hyperbolic. Recall that two metric spacs $\left(X, d_{X}\right)$ and $\left(Y, d_{Y}\right)$ are quasiisometric if there exist $a>0, a^{\prime}>0, b, b^{\prime} \in \mathbf{R}$ and a map $f: Y \rightarrow X$ such that for every $y, y^{\prime} \in Y$, we have

$$
a d\left(y, y^{\prime}\right)-b \leq d\left(f(y), f\left(y^{\prime}\right)\right) \leq a^{\prime} d\left(y, y^{\prime}\right)+b^{\prime} .
$$

Let us define a map

$$
f: \mathcal{G}(\Gamma, S) \rightarrow X
$$

as follows. The map $f$ sends homothetically the edges $\left[e, \sigma_{i}\right]$ in the Cayley graph onto geodesic segments $\left[x, \sigma_{i} x\right]$ in $X$, (recall that the edges in the Cayley graph have length equal to 1 , hence the homothety factors are the length of the geodesic segments $\left[x, \sigma_{i} x\right]$, that is, the distances $\left.d\left(x, \sigma_{i} x\right)\right)$. We then define $f$ on every edge by $f\left(\left[\gamma, \gamma \sigma_{i}\right]\right)=\gamma\left[x, \sigma_{i} x\right]$. The first observation is that the map $f:\left(\mathcal{G}(\Gamma, S), d_{S}\right) \rightarrow(X, d)$ is a quasiisometry: for every $s, t \in \mathcal{G}(\Gamma, S)$,

$$
4 D d_{S}(s, t)-14 D \leq d(f(s), f(t)) \leq 6 D d_{S}(s, t) .
$$

We skip the proof of (5), which is a quite direct consequence of the definition of $f$, and refer to [BCGS20a], Lemma 5.7, for details.

The second observation below is a direct consequence of (5): given a geodesic segment $[s, t] \subset \mathcal{G}(\Gamma, S)$ between $s, t$, we have

$$
\operatorname{Length}(f[s, t]) \leq \frac{3}{2} d(f(s), f(t))+21 D .
$$


Indeed, $f([s, t])$ is arcwise geodesic in $X$; thus, by the second inequality of $(5)$, we get Length $f([s, t]) \leq 6 D d_{S}(s, t)$, and (6) follows from the first inequality of (5).

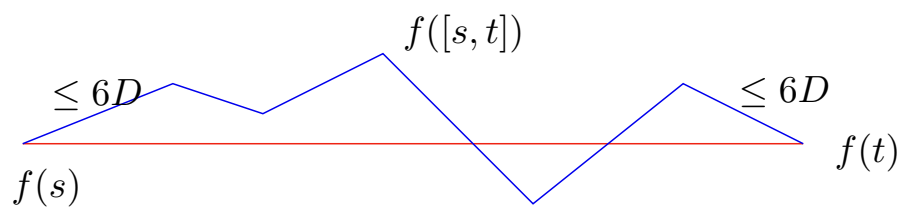

Figure 7.2: Length $f([s, t])$

We now recall a fundamental property of $\delta$-hyperbolic spaces which amounts to saying that if a curve $c$ satisfies the property that the length of every subsegment of $c$ is equal to the distance between its end points up to multiplicative and additive constants, then $c$ is at bounded distance from each geodesic joining the end points.

Lemma 7.5 ([BCGS20a], Proposition 6.13). Let $(X, d)$ be a $\delta$-hyperbolic space and $c:[0, T] \rightarrow X$ a rectifiable curve such that for every $u, v \in[0, T]$,

$$
\operatorname{Length}(c([u, v]) \leq \lambda d(c(u), c(v))+C .
$$

Then, every geodesic in $X$ joining $c(0)$ and $c(T)$ is contained in the $C_{1}$-neighborhood of $c([0, T])$ and conversely, $c([0, T])$ is contained in the $C_{2}$-neighborhood of each geodesic between $c(0)$ and $c(T)$, where $C_{1}:=6 \lambda+2+\frac{C}{6 \lambda+2}$ and $C_{2}:=(1+\lambda) C_{1}+\frac{C}{2}$.

We can apply Lemma 7.5 to the image $c:=f\left(\left[y, y^{\prime}\right]\right)$ by $f$ of geodesic segments $\left[y, y^{\prime}\right]$ in $\mathcal{G}(\Gamma, S)$. Let $\bar{c}$ be a geodesic joining $f(y)$ and $f\left(y^{\prime}\right)$. By the observation (6), the hypothesis of Lemma 7.5 is satisfied with $\lambda=\frac{3}{2}$ and $C=21 D$, therefore the curve $c:=f\left(\left[y, y^{\prime}\right]\right)$ is contained in the $C_{1}:=11 \delta+\frac{21 D}{11}$-neighborhood of the geodesic $\bar{c}$ and $\bar{c}$ is in the $C_{2}$-neighbourhood of $c$ for $C_{2}:=\frac{55 \delta}{2}+\frac{168 D}{11}$.

We are now ready to conclude. Let $\left(y_{0}, y_{1}, y_{2}\right)$ be a geodesic triangle in $\mathcal{G}(\Gamma, S)$. We want to show that $\left(y_{0}, y_{1}, y_{2}\right)$ is $\frac{\delta^{\prime}}{4}$-thin, i.e. that every point $u$ on one side of $\left(y_{0}, y_{1}, y_{2}\right)$ is at distance $\frac{\delta^{\prime}}{4}$ of the union of the two other sides of the triangle. For every $i \neq j \in\{0,1,2\}$, we choose $\bar{c}_{i, j}$ a geodesic joining $f\left(y_{i}\right)$ and $f\left(y_{j}\right)$ and denote by $\left(f\left(y_{0}\right), f\left(y_{1}\right), f\left(y_{2}\right)\right)$ the geodesic triangle in $X$ whose sides are the $\bar{c}_{i, j}$ 's. We can assume that $u \in\left[y_{1}, y_{2}\right]$. By Lemma 7.5 there exists $v \in \bar{c}_{1,2}$ such that $d(f(u), v) \leq C_{1}$. Since $(X, d)$ is $\delta$-hyperbolic, there exists $v^{\prime} \in \bar{c}_{0,1} \cup \bar{c}_{0,2}$ such that $d\left(v, v^{\prime}\right) \leq \delta$. We can assume that $v^{\prime} \in \bar{c}_{0,1}$. By Lemma 7.5, there exists $f\left(u^{\prime}\right) \in f\left(\left[y_{0}, y_{1}\right]\right)$ such that $d\left(f\left(u^{\prime}\right), v^{\prime}\right) \leq C_{2}$. By (5) we have $d\left(u, u^{\prime}\right) \leq\left(\frac{1}{4 D} d\left(f(u), f\left(u^{\prime}\right)\right)+\frac{14 D}{4 D}\right)$, hence $d\left(u, u^{\prime}\right) \leq \frac{1}{4 D}\left(d(f(u), v)+d\left(v, v^{\prime}\right)+d\left(v^{\prime}, f\left(u^{\prime}\right)\right)\right)+$ $\frac{14 D}{4 D}$, and therefore,

$$
d\left(u, u^{\prime}\right) \leq \frac{1}{4 D}\left(C_{1}+\delta+C_{2}\right)+\frac{14 D}{4 D},
$$

thus, $d\left(u, u^{\prime}\right) \leq \frac{1}{4 D}(40 \delta+32 D)$. We conclude that $\mathcal{G}(\Gamma, S)$ is $\delta^{\prime}:=8\left(\frac{5 \delta}{D}+4\right)$-hyperbolic. 


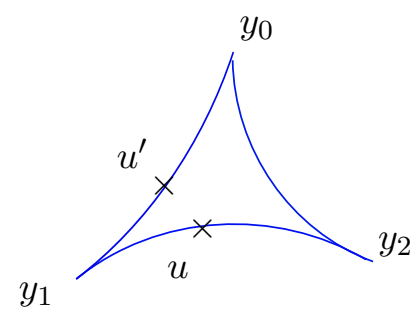

$\mathcal{G}(\Gamma, S)$
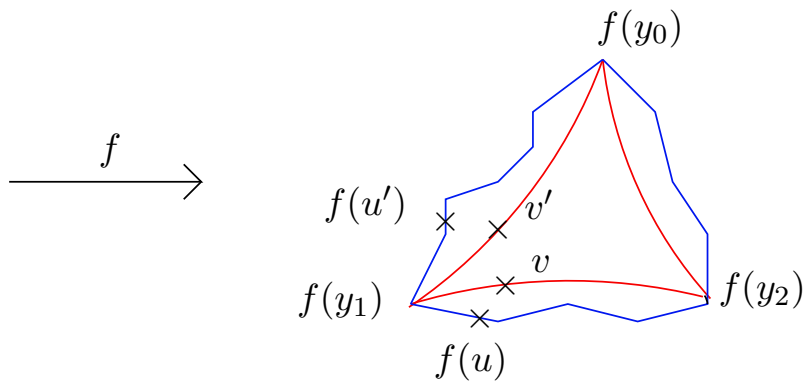

$f\left(y_{1}\right)$

$f(u)$

$X$

Figure 7.3: $\mathcal{G}(\Gamma, S)$ is $\delta^{\prime}$-hyperbolic

\subsection{Finitely presented groups}

Let us consider a marked group $(\Gamma, S)$ with its marking $\varphi_{\Gamma, S}: \mathbf{F}_{k} \rightarrow \Gamma$. We then have $\Gamma \simeq \mathbf{F}_{k} / \operatorname{Ker} \varphi_{\Gamma, \mathrm{S}}$.

Definition 7.6. A group $\Gamma$ is finitely presented if there exists a finite generating set $S$ such that $\operatorname{Ker} \varphi_{\Gamma, \mathrm{S}}=\left\langle\left\langle\mathrm{r}_{1}, \cdots, \mathrm{r}_{\mathrm{n}}\right\rangle\right\rangle$ is finitely generated as a normal subgroup. We write

$$
\Gamma=\left\langle e_{1}, \cdots, e_{k} \mid r_{1}, \cdots, r_{n}\right\rangle \text {. }
$$

Notice that when $\Gamma=\left\langle e_{1}, \cdots, e_{k} \mid r_{1}, \cdots, r_{n}\right\rangle$, then we have $\Gamma \simeq \mathbf{F}_{k} /\left\langle\left\langle r_{1}, \cdots, r_{n}\right\rangle\right\rangle$.

Examples 7.7. 1. $\mathbf{Z}^{2}=\left\langle a, b \mid a b a^{-1} b^{-1}\right\rangle$.

2. If $M$ is a genus $g$ surface, $g \geq 2$, then

$$
\pi_{1}(M)=\left\langle a_{1}, b_{1}, \cdots, a_{g}, b_{g} \mid \Pi_{i=1}^{g}\left[a_{i}, b_{i}\right]\right\rangle .
$$

We set

$$
\mathbf{B}_{p}(3):=\left\{g \in \mathbf{F}_{p} \mid d_{S_{p}}(e, g)=2, \text { or } 3\right\},
$$

where $d_{S_{p}}$ is the distance on the Cayley graph of the non abelian free group $\mathbf{F}_{p}$ on $p$ generators and $S_{p}:=\left\{e_{1}^{ \pm 1}, \cdots, e_{p}^{ \pm 1}\right\}$ the associated generating set.

The next classical proposition says that if $(\Gamma, S)$ is a $\delta$-hyperbolic group, then there exists a presentation of $\Gamma$ with relations being words of length less than or equal to 3 . Before stating it, let us define, for every $\delta$-hyperbolic group $(\Gamma, S)$, the "augmented generating set" as follows. 
Definition 7.8. $\Sigma_{S}:=\left\{\gamma \in \Gamma \backslash\{e\} \mid d_{S}(e, \gamma)<4 \delta+2\right\}$.

Notice that $\Sigma_{S}$ is a generating set of $\Gamma$ since $S \subset \Sigma_{S}$.

Proposition 7.9 ([CDP90], Theorem 2.5, Chapter 5). Let $(\Gamma, S)$ be a $\delta$-hyperbolic group and $\Sigma_{S}$ the augmented generating set. We set $p:=\# \Sigma_{S}$ and $\mathcal{R}_{\Gamma, \Sigma_{S}}:=\operatorname{Ker} \varphi_{\Gamma, \Sigma_{S}} \cap \mathbf{B}_{\mathrm{p}}(3)$. Then, $\operatorname{Ker} \varphi_{\Gamma, \Sigma_{\mathrm{S}}}=\left\langle\left\langle\mathcal{R}_{\Gamma, \Sigma_{\mathrm{S}}}\right\rangle\right\rangle$, in particular $\Gamma$ is finitely presented.

Remark 7.10. By definition of $\Sigma_{S}, p:=\# \Sigma_{S}$ is bounded above in term of $\# S$ and $\delta$, hence by the above proposition the same is true for the number of relations $\# \mathcal{R}_{\Gamma, \Sigma_{S}}$ of the presentation of $\left(\Gamma, \Sigma_{S}\right)$. This fact will be used to show that the number of isomorphism classes of marked groups such as $(\Gamma, S)$ is bounded above by a function depending on \#S and $\delta$.

We now define the family of $\delta$-hyperbolic groups having a generating set with bounded cardinality. In what follows, we fix $\delta>0$.

Definition 7.11. We define $M G_{k}$ as the class of marked group $(\Gamma, S)$ such that $(\Gamma, S)$ is $\delta$-hyperbolic and $\# S \leq k$.

Since we are interested in groups up to isomorphism, we consider the following

Definition 7.12. We define $G_{k}$ as the set of groups $\Gamma$ such that there exists a generating set $S$ with $(\Gamma, S) \in M G_{k}$.

Recall that we write $(\Gamma, S) \simeq\left(\Gamma^{\prime}, S^{\prime}\right)$ when the marked groups $(\Gamma, S)$ and $\left(\Gamma^{\prime}, S^{\prime}\right)$ are isomorphic, cf. Definition 7.2. We will also write $\Gamma \sim \Gamma^{\prime}$ when $\Gamma$ and $\Gamma^{\prime}$ are isomorphic. The set of corresponding isomorphism classes are denoted $M G_{k} / \simeq$ and $G_{k} / \sim$ repectively.

Proposition 7.13. The set of isomorphism classes $G_{k} / \sim$ contains less than

$$
M(k):=2^{N_{k}} \sum_{p=3}^{N_{k}} 2^{4 p^{2}(2 p-1)}
$$

elements, where $N_{k}:=2 k(k-1)^{\lfloor 4 \delta+1\rfloor}$.

Proof. We sketch the proof; the details can be found in [BCGS20a], Proposition 5.9. It follows from the definitions that the map from $M G_{k}$ onto $G_{k}$ defined by $(\Gamma, S) \longmapsto \Gamma$ induces a surjective map $M G_{k} / \simeq \longrightarrow G_{k} / \sim$, therefore

$$
\#\left(G_{k} / \sim\right) \leq \#\left(M G_{k} / \simeq\right) .
$$

Proposition 7.13 then amounts to finding a bound of $\#\left(M G_{k} / \simeq\right)$. In order to find such a bound, we will construct a finite to one map from $M G_{k} / \simeq$ to a finite set.

We first notice that for every $(\Gamma, S) \in M G_{k}$ the cardinality of the generating set $\Sigma_{S}$ introduced in Definition 7.8 is bounded above by the number of elements of the ball of 
radius $\lfloor 4 \delta+2\rfloor$ of the Cayley graph of the non abelian free group $\mathbf{F}_{k}$ with respect to the standard set of generators. Therefore,

$$
\# \Sigma_{S} \leq 1+\sum_{i=1}^{\lfloor 4 \delta+2\rfloor} k(k-1)^{i-1} \leq N_{k}:=2 k(k-1)^{\lfloor 4 \delta+1\rfloor}
$$

since $1+X+\cdots+X^{n} \leq 2 X^{n}-1$ and by $(10)$, the marked group $\left(\Gamma, \Sigma_{S}\right)$ then belongs to $M G_{N_{k}}$.

We now give several claims which will lead to the construction of a map from $M G_{k} / \simeq$ to a finite set. We will then sketch the proofs of the claims.

Claim 7.14. The map $\psi: M G_{k} \rightarrow M G_{N_{k}}$, defined by $\psi((\Gamma, S)):=\left(\Gamma, \Sigma_{S}\right)$, induces a $\operatorname{map} \bar{\psi}: M G_{k} / \simeq \rightarrow M G_{N_{k}} / \simeq$.

For every $(\Gamma, \Sigma) \in M G_{N_{k}}$ we set $p(\Sigma):=\# \Sigma$. Recall that $\mathcal{R}_{\Gamma, \Sigma}=\operatorname{Ker} \varphi_{\Gamma, \Sigma} \cap \mathbf{B}_{\mathrm{p}(\Sigma)}(3)$, where $\varphi_{\Gamma, \Sigma}$ is the marking of $(\Gamma, \Sigma)$. For $p \in \mathbf{N}$, we denote by $\mathcal{A}(p)$ the set of all subsets of $\mathbf{B}_{p}(3)$, where $\mathbf{B}_{p}(3)$ has been defined in (8) and we set

$$
\mathcal{A}:=\cup_{p=3}^{N_{k}}\{p\} \times \mathcal{A}(p) .
$$

Claim 7.15. The map $\Phi: M G_{N_{k}} \rightarrow \mathcal{A}$ defined by $\Phi((\Gamma, \Sigma)):=\left(p(\Sigma), \mathcal{R}_{\Gamma, \Sigma}\right)$ induces a map $\bar{\Phi}: M G_{N_{k}} / \simeq \rightarrow \mathcal{A}$. Moreover, $\bar{\Phi}_{\mid \operatorname{Im} \bar{\psi}}$ is injective, where $\bar{\Phi}_{\mid \operatorname{Im} \bar{\psi}}$ denotes the restriction of $\bar{\Phi}$ to $\operatorname{Im} \bar{\psi}=\bar{\psi}\left(\mathrm{MG}_{\mathrm{k}} / \simeq\right)$.

By claims 7.14 and 7.15, the map $\Phi \circ \psi: M G_{k} \stackrel{\psi}{\rightarrow} M G_{N_{k}} \stackrel{\Phi}{\rightarrow} \mathcal{A}$ induces a composed $\operatorname{map} \bar{\Phi} \circ \bar{\psi}$,

$$
M G_{k} / \simeq \stackrel{\bar{\psi}}{\rightarrow} M G_{N_{k}} / \simeq \stackrel{\bar{\Phi}}{\rightarrow} \mathcal{A} .
$$

We now show that the map $\bar{\Phi} \circ \bar{\psi}: M G_{k} / \simeq \rightarrow \mathcal{A}$ is finite to one.

We observe first that for every $(\Gamma, \Sigma) \in M G_{N_{k}}$, we have

$$
\psi^{-1}(\{(\Gamma, \Sigma)\})=\left\{(\Gamma, S) \in M G_{k} \mid \Sigma_{S}=\Sigma\right\}
$$

hence,

$$
\psi^{-1}(\{(\Gamma, \Sigma)\}) \subset\left\{(\Gamma, S) \in M G_{k} \mid S \subset \Sigma\right\} .
$$

Therefore, $\# \psi^{-1}((\Gamma, \Sigma)) \leq 2^{N_{k}}$.

We now denote by $[(\Gamma, \Sigma)]$ the isomorphism class of $(\Gamma, \Sigma) \in M G_{N_{k}}$, and we choose some $\left(\Gamma^{\prime}, S^{\prime}\right) \in M G_{k}$ such that

$$
\left[\left(\Gamma^{\prime}, S^{\prime}\right)\right] \in \bar{\psi}^{-1}(\{[(\Gamma, \Sigma)]\}) .
$$

By definition, $\psi\left(\left(\Gamma^{\prime}, S^{\prime}\right)\right)=\left(\Gamma^{\prime}, \Sigma_{S^{\prime}}\right)$ is isomorphic to $(\Gamma, \Sigma)$. The isomorphism

$$
\beta:\left(\Gamma^{\prime}, \Sigma_{S^{\prime}}\right) \rightarrow(\Gamma, \Sigma)
$$

sends $\left(\Gamma^{\prime}, S^{\prime}\right)$ on $(\Gamma, S)$ for some $S \subset \Sigma$ such that $\Sigma=\Sigma_{S}$ thus, $(\Gamma, S) \in \psi^{-1}(\{(\Gamma, \Sigma)\})$. We have shown that each isomorphism class in $\bar{\psi}^{-1}(\{[(\Gamma, \Sigma)]\})$ contains a representative $(\Gamma, S)$ in $\psi^{-1}(\{(\Gamma, \Sigma)\})$, hence, $\#\left(\bar{\psi}^{-1}(\{[(\Gamma, \Sigma)]\})\right) \leq \#\left(\psi^{-1}(\{(\Gamma, \Sigma)\})\right) \leq 2^{N_{k}}$. 


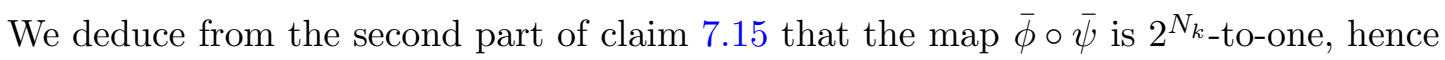

$$
\#\left(M G_{k} / \simeq\right) \leq 2^{N_{k}} \# \mathcal{A}=M(k),
$$

since $\# \mathcal{A}=\sum_{p=3}^{N_{k}} 2^{4 p^{2}(2 p-1)}$.

It remains to sketch the proof of the claims. The claim 7.14 is a direct consequence of the fact that an isomorphism between $(\Gamma, S)$ and $\left(\Gamma^{\prime}, S\right)$ induces an isometry between the corresponding Cayley graphs of $(\Gamma, S)$ and $\left(\Gamma^{\prime}, S^{\prime}\right)$. In order to prove the first part of the claim 7.15 , we consider two isomorphic marked groups $(\Gamma, \Sigma)$ and $\left(\Gamma^{\prime}, \Sigma^{\prime}\right)$ with marking $\varphi_{\Gamma, \Sigma}$ and $\varphi_{\Gamma^{\prime}, \Sigma^{\prime}}$ respectively. We observe that $\varphi_{\Gamma^{\prime}, \Sigma}=\beta \circ \varphi_{\Gamma, \Sigma}$ where $\beta$ is the isomorphism between $(\Gamma, \Sigma)$ and $\left(\Gamma^{\prime}, \Sigma^{\prime}\right)$. This implies that $p(\Sigma)=p\left(\Sigma^{\prime}\right)$ and that $\operatorname{Ker} \varphi_{\Gamma, \Sigma}=\operatorname{Ker} \varphi_{\Gamma^{\prime}, \Sigma^{\prime}}$, therefore $\mathcal{R}_{\Gamma, \Sigma}=\mathcal{R}_{\Gamma^{\prime}, \Sigma^{\prime}}$ and $\Phi((\Gamma, \Sigma))=\Phi\left(\left(\Gamma^{\prime}, \Sigma^{\prime}\right)\right)$. This concludes the proof of the claims. Proposition 7.13 follows from (9) and (11).

\subsection{Finiteness Theorem}

In this section we state a Finiteness Theorem. We will assume that the metric space $(X, d)$ is geodesically complete, non elementary $\delta$-hyperbolic and Busemann. Let $\mathcal{H}(\delta, H, D)$ be the set of torsion free groups $\Gamma$ which admit a proper and discontinuous action on a metric space satisfying the above properties and such that diam $X / \Gamma \leq D$ and $\operatorname{Ent}(X, d) \leq H$. The set of isomorphism classes of groups in $\mathcal{H}(\delta, H, D)$ is denoted by $\mathcal{H}(\delta, H, D) / \sim$.

A first step toward the finiteness theorem is to show that every group $\Gamma \in \mathcal{H}(\delta, H, D)$ has a generating set with bounded cardinal. We first recall the following property from Lecture 5, Proposition 5.13.

Proposition 7.16. Let $(X, d)$ be a non elementary $\delta$-hyperbolic space and a group $\Gamma$ acting properly by isometries on $(X, d)$. Assume that $\operatorname{diam}(X / \Gamma) \leq D$ and $\operatorname{Ent}(X, d) \leq$ $H$. Then there exists $x_{0} \in X$ such that

$$
\operatorname{sys}_{\Gamma}\left(x_{0}\right) \geq \epsilon_{0}(\delta, H, D) .
$$

Theorem 7.17. Given $\delta, H, D$, let $\epsilon_{0}:=\epsilon_{0}(\delta, H, D)$ be as in Proposition 7.16 and let $\Gamma$ be a torsion free group acting properly on $(X, d)$, a non elementary $\delta$-hyperbolic Busemann space. Then, there exists $x_{0} \in X$ such that

$$
\# S_{6 D}\left(x_{0}\right) \leq n_{0}(\delta, H, D)
$$

where $n_{0}(\delta, H, D):=\frac{11}{2} 3^{\frac{25}{4}}\left(\frac{6 D}{\epsilon_{0}}\right)^{\frac{25}{4}} \exp \left(18 H(11 D+20 \delta) \frac{6 D}{\epsilon_{0}}\right)$.

Proof. Let $x_{0} \in X$ be as in Proposition 7.16. In particular, for every $\gamma \in \Gamma \backslash\{I d\}$, we have

$$
d\left(x_{0}, \gamma x_{0}\right) \geq \epsilon_{0}(\delta, H, D) .
$$

It follows from (12) that $\mu_{x_{0}}^{\Gamma}\left(B\left(x_{0}, \epsilon_{0}\right)\right)=1$. Applying the Bishop-Gromov Theorem for small radii, Theorem 4.3 with $(r, R)$ replaced by $\left(\epsilon_{0}, 6 D\right)$, we then deduce

$$
\# S_{6 D}\left(x_{0}\right)=\frac{\mu_{x_{0}}^{\Gamma}\left(B\left(x_{0}, 6 D\right)\right)}{\mu_{x_{0}}^{\Gamma}\left(B\left(x_{0}, \epsilon_{0}\right)\right)} \leq n_{0}(\delta, H, D) .
$$


Theorem 7.18. $\mathcal{H}(\delta, H, D) / \sim$ contains less than $M_{0}(\delta, H, D)=2^{N_{0}} \sum_{p=1}^{N_{0}} e^{4 p^{2}(p-1)}$ elements, where $N_{0}=2 n_{0}\left(n_{0}-1\right)^{\left(\frac{40 \delta}{D}+35\right)}$

Proof. Let us choose $x_{0} \in X$ such as in Theorem 7.17,

$$
\# S_{6 D}\left(x_{0}\right) \leq n_{0}:=n_{0}(\delta, H, D),
$$

where

$$
n_{0}(\delta, H, D)=\frac{11}{2} 3^{\frac{25}{4}}\left(\frac{6 D}{\epsilon_{0}}\right)^{\frac{25}{4}} \exp \left(18 H(11 D+20 \delta) \frac{6 D}{\epsilon_{0}}\right) .
$$

By Proposition 7.4, $S_{6 D}\left(x_{0}\right)$ is a generating set of $\Gamma$ and $\left(\Gamma, S_{6 D}\left(x_{0}\right)\right)$ is $\delta^{\prime}$-hyperbolic where $\delta^{\prime}=8\left(\frac{5 \delta}{D}+4\right)$ therefore, $\left(\Gamma, S_{6 D}\left(x_{0}\right)\right) \in M G_{n_{0}}$, where we have replaced $\delta$ by $\delta^{\prime}$ in the definition of $M G_{n_{0}}$. Proposition 7.13 then implies that there exist less than $M\left(n_{0}\right)$ such groups $\Gamma$, where

$$
M\left(n_{0}\right)=2^{N_{n_{0}}} \sum_{p=3}^{N_{n_{0}}} 2^{4 p^{2}(2 p-1)},
$$

and

$$
N_{n_{0}}:=2 n_{0}\left(n_{0}-1\right)^{\left\lfloor 4 \delta^{\prime}+1\right\rfloor}=2 n_{0}\left(n_{0}-1\right)^{\left\lfloor\frac{160 \delta}{D}+129\right\rfloor},
$$

which concludes.

\section{Lecture 8}

In this lecture we intend to discuss some homotopical finiteness theorem. We need to define a new property for a proper geodesic metric space $(X, d)$ using triangle comparison as in figure 1.3 reproduced below.

\subsection{CAT(0)-spaces}

We consider, as in Lecture 1, a triangle $\Delta \subset X$ and its comparison triangle $\bar{\Delta} \subset \mathbf{R}^{2}$.

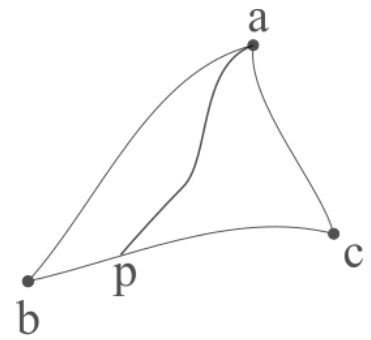

$\Delta$

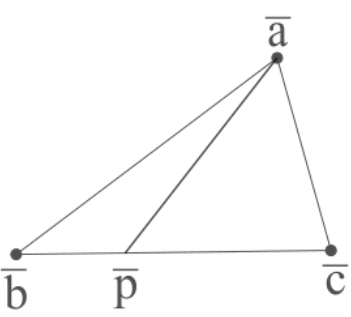

$\bar{\Delta}$

Figure 8.1: $\Delta \subset X$ and $\bar{\Delta} \subset \mathbf{R}^{2}$ 
We choose a point $p$ on the segment $[b, c]$ and a point $\bar{p}$ on the segment $[\bar{b}, \bar{c}]$ dividing it in the same ratio, that is,

$$
d(b, p)=t d(b, c) \text { and } \bar{d}(\bar{b}, \bar{p})=t \bar{d}(\bar{b}, \bar{c}) \text { for } t \in[0,1] .
$$

Definition 8.1. The space $(X, d)$ is said to be $\mathrm{CAT}(0)$ if for every triangle $\Delta$ and every such point $p \in \Delta$ we have,

$$
d(a, p) \leq \bar{d}(\bar{a}, \bar{p}) .
$$

Remarks 8.2. $\quad$ i) The interested reader is referred to [BH99] for a full treatment of the notion of $\mathrm{CAT}(\kappa)$-spaces for $\kappa \in \boldsymbol{R}$.

ii) Contrarily to $\delta$-hyperbolicity, the $\mathrm{CAT}(0)$ property imposes some local restrictions.

Exercises 8.3. 1. Show that CAT(0)-spaces are Busemann. The converse is not true in general, see [BH99] p.169.

2. Show that a $\mathrm{CAT}(-1)$-space is $\delta$-hyperbolic and compute $\delta$.

Notice that in a CAT(0)-space metric balls are contractible. We can also localise this notion, more precisely

Definition 8.4. The space $(X, d)$ is said to be locally CAT(0) if for all $x \in X$, there exists a neighbourhood $U_{x}$ of $x$ such that every triangle included in $U_{x}$ satisfies the $\mathrm{CAT}(0)$ property.

Let us recall that we always assume that $(X, d)$ satisfies the property of extension of geodesics. We then get

Lemma 8.5 (see [BH99]). Every locally CAT(0) metric space admits a universal cover which is $\mathrm{CAT}(0)$ and is geodesically complete.

Let us now choose three real numbers $\delta \geq 0, H>0$ and $D>0$.

Definition 8.6. We consider compact, connected, metric spaces $(Y, d)$ which are locally $\operatorname{CAT}(0)$, with $\operatorname{diam}(Y, d) \leq D$, and which have a $\delta$-hyperbolic universal cover whose entropy is not greater than $H$. We denote this space by $\mathcal{M}(\delta, H, D)$.

The following theorem is an easy consequence of Theorem 7.18.

Theorem 8.7. With the above notations, we have the following.

i) The number of fundamental groups, up to isomorphisms, of elements of $\mathcal{M}(\delta, H, D)$ is bounded above by $M_{0}(\delta, H, D)$.

ii) The number of elements of $\mathcal{M}(\delta, H, D)$, up to homotopy equivalence, is bounded above by $M_{0}(\delta, H, D)$.

The function $M_{0}(\delta, H, D)$ is described more precisely in Theorem 7.18. 
Proof. We just have to check that the hypotheses of Theorem 7.18 are satisfied.

i) The fundamental groups of elements of $\mathcal{M}(\delta, H, D)$ are in $\mathcal{H}(\delta, H, D)$, the class defined in Subsection 7.3. Notice that, on a $\mathrm{CAT}(0)$-space which is Busemann, the isometries in a fundamental group are torsion-free, see Remark 6.3.

ii) For $(Y, d) \in \mathcal{M}(\delta, H, D)$, its universal cover $\widetilde{Y}$ is contractible, hence it is a $K(\pi, 1)$. This implies that, for $Y, Y^{\prime} \in \mathcal{M}(\delta, H, D)$, having an isomorphism between $\pi_{1}(Y)$ and $\pi_{1}\left(Y^{\prime}\right)$ is equivalent to having an homotopy equivalence between $Y$ and $Y^{\prime}$. We recall that a homotopy equivalence between $Y$ and $Y^{\prime}$ is equivalent to having two continuous maps $f: Y \longrightarrow Y^{\prime}$ and $g: Y^{\prime} \longrightarrow Y$ such that $g \circ f$ is homotopic to the identity map on $Y$ and $f \circ g$ is homotopic to the identity map on $Y^{\prime}$.

Remark 8.8. Theorem 8.7 is certainly true when the hypothesis "locally CAT(0)" is replaced by the hypothesis "locally Busemann". However, we have not yet done the proof.

\subsection{Towards a compactness result}

The aim of the next step is to endow $\mathcal{M}(\delta, H, D)$ with a distance function, $d_{G H}$ and show that the metric space $\left(\mathcal{M}(\delta, H, D), d_{G H}\right)$ is compact. Let us first recall a few facts about the Gromov-Hausdorff distance between metric spaces.

\subsubsection{Gromov-Hausdorff distance between metric spaces}

Let $(X, d)$ be a general metric space and $A, B \subset X$ two compact subsets. We define the Hausdorff distance $d_{X, H}(A, B)$ between subsets $A$ and $B$ of $X$ by,

$$
d_{X, H}(A, B)=\inf \left\{\epsilon>0 ; A \subset B_{\epsilon} \text { and } B \subset A_{\epsilon}\right\},
$$

where $A_{\epsilon}=\{x \in X ; d(x, A) \leq \epsilon\}$.

Now let $\left(X, d_{X}\right)$ and $\left(Y, d_{Y}\right)$ be two compact metric spaces. We consider metric spaces $\left(Z, d_{Z}\right)$ such that there exist two isometric embeddings,

$$
i: X \longrightarrow Z \text { and } j: Y \longrightarrow Z
$$

and define

$$
d_{G H}(X, Y)=\inf _{Z, i, j}\left\{d_{Z, H}(i(X), j(Y))\right\} .
$$

Here $d_{Z, H}$ is the Hausdorff distance between compact subsets of $Z$.

Exercise 8.9. Prove that such spaces $Z$ always exist and that $d_{G H}$ is a distance.

The strength of this definition is clear in the following proposition

Proposition 8.10. The set $\mathcal{M}$ of compact length spaces (modulo isometries) endowed with $d_{G H}$ is complete. 
Remarks 8.11. 1) Notice that $\mathcal{M}$ is a set.

2) This proposition is left to the reader. Completeness in this situation is tricky but not difficult.

Obviously this definition is not really convenient since we have to know all possible metric spaces $Z$. We thus turn to the standard more effective alternative approach.

Definitions 8.12. An $\epsilon$-approximation between two metric spaces $\left(X, d_{X}\right)$ and $\left(Y, d_{Y}\right)$ is given by two maps $\varphi: X \rightarrow Y$ and $\psi: Y \rightarrow X$ which are $\epsilon$-almost reciprocal, i.e. such that $d_{Y}(y, \varphi \circ \psi(y))<\epsilon$ and $d_{X}(x, \psi \circ \varphi(x))<\epsilon$ for all $(x, y) \in X \times Y$ and which furthermore satisfy:

$\forall x, x^{\prime} \in X \quad\left|d_{Y}\left(\varphi(x), \varphi\left(x^{\prime}\right)\right)-d_{X}\left(x, x^{\prime}\right)\right|<\epsilon ; \quad \forall y, y^{\prime} \in Y\left|d_{X}\left(\psi(y), \psi\left(y^{\prime}\right)\right)-d_{Y}\left(y, y^{\prime}\right)\right|<\epsilon$.

A sequence $\left(\left(X_{n}, d_{n}\right)\right)_{n \in \mathbf{N}}$ is said to "converge in the Gromov-Hausdorff sense to $(X, d)$ " if there exists a sequence of positive numbers $\left(\epsilon_{n}\right)_{n \in \mathbf{N}}$ approching zero and, for every $n$, an $\epsilon_{n}$-approximation between $(X, d)$ and $\left(X_{n}, d_{n}\right)$.

Remarks 8.13. 1. This notion of convergence is equivalent to convergence under the Gromov-Hausdorff distance defined before up to multiplicative factors of $\epsilon_{n}$.

2. The reader may also check yet another definition closest to the one given by Gromov, in [BH99], page 72.

We now define the notion of $\epsilon$-net.

Definition 8.14. An $\epsilon$-net $Z \subset X$ of a compact metric space $\left(X, d_{X}\right)$ is a finite set of points $\left\{x_{i}\right\}_{i \in I}$ of $X$ such that $\bigcup_{i \in I} B\left(x_{i}, \epsilon\right)=X$.

Proposition 8.15. Let $D>0$ and $N:(0,1) \longrightarrow N$ an integer valued map. We denote by $\operatorname{Met}(D, N)$ the set of all isometry classes of compact metric spaces satisfying

1. $\operatorname{diam}(M) \leq D$,

2. $\forall \epsilon, \exists Z$ a finite $\epsilon$-net such that $\# Z \leq N(\epsilon)$.

Then, $\operatorname{Met}(D, N)$ is compact for the distance $d_{G H}$.

This result is classical and not difficult, just a bit boring to describe. We then get the following theorem.

Theorem 8.16 ([Gro07], Theorem 5.3, p.275). Let Man $(n, D)$ be the set of isometry classes of closed Riemannian manifolds $(M, g)$ of dimension $n$ such that $\operatorname{Ric}(g) \geq-(n-$ 1) and diameter not greater than $D$. Then, $\operatorname{Man}(n, D)$ is relatively compact in the space of all isometry classes of compact metrics spaces.

Remark 8.17. The proof consists in showing that $\mathcal{M a n}(n, D) \subset \mathcal{M e t}(D, N)$. However it is not closed, which explains that the conclusion is only "relatively compact". 
Proof. We need to show that, for every $\epsilon>0$, there exists an $\epsilon$-net with a uniformly bounded cardinality, independently of $(M, g) \in \operatorname{Man}(n, D)$.

Now, for $(M, g) \in \operatorname{Man}(n, D)$, let $\epsilon>0$ and choose $Z \subset M$ to be a maximal subset satisfying that if $z_{1}, z_{2} \in Z, z_{1} \neq z_{2}$ then $d_{g}\left(z_{1}, z_{2}\right)>\epsilon$, where $d_{g}$ denotes the distance in $M$ induced by the metric $g$. This is a maximal packing by balls of radius $\epsilon / 2$. Notice that there might be several subset $Z$ and we choose one. The balls $B(z, \epsilon / 2) \subset M$, for $z \in Z$ are disjoints, hence, for $m \in M$,

$$
\sum_{z \in Z} \operatorname{Vol}(B(z, \epsilon / 2)) \leq \operatorname{Vol}(M)=\operatorname{Vol}(B(m, D)) .
$$

As a consequence,

$$
\# Z \leq \frac{\operatorname{Vol}(M)}{\inf _{z \in Z}\{\operatorname{Vol}(B(z, \epsilon / 2))\}}=\frac{\operatorname{Vol}\left(B\left(z_{0}, D\right)\right)}{\operatorname{Vol}\left(B\left(z_{0}, \epsilon / 2\right)\right)}=\frac{\operatorname{Vol}_{-1}(D)}{\operatorname{Vol}_{-1}(\epsilon / 2)}:=N(\epsilon) .
$$

Here $\operatorname{Vol}_{-1}(r)$ denotes the volume of a ball of radius $r$ in a simply connected real hyperbolic space of constant sectional curvature equal to -1 . This ends the proof of Theorem 8.16 .

We finish this lecture by stating one of the main theorems of [BCGS20a].

Theorem 8.18 (see $[\mathrm{BCGS20a}])$. The metric space $\left(\mathcal{M}(\delta, D, H), d_{G H}\right)$ is compact.

Remarks 8.19. $\quad$ 1. Notice that in the last theorem, contrarily to Theorem 8.16, there is no reference to any kind of dimension of the metric spaces in $\mathcal{M}(\delta, D, H)$. However the core of the proof will show that the bound on the dimension is hidden in the bound on $H$.

2. This result is certainly true with the hypothesis "Busemann" instead of "CAT(0)".

\section{Lecture 9}

In the first part of this lecture, we state a compactness Theorem for the set of isometry classes of locally $\mathrm{CAT}(0)$ metric spaces with bounded diameter and universal cover $\delta$ hyperbolic with bounded entropy. When the metric spaces are assumed to be topological manifolds, this leads to a topological finiteness Theorem. In the second part of the lecture, we come back to the growth of groups and raise a few questions that we will discuss in the last lecture.

\subsection{Compactness}

We define $\mathcal{M}:=\{(Y, d) \mid(Y, d)$ compact metric space $\}$. In this section, for every metric space $(Y, d)$, we will denote by $(X, d)$ its universal cover. In particular, we will not distinguish the corresponding distances. We denote by $\mathcal{M}(\delta, H)$ the set of connected, 
compact, locally $\mathrm{CAT}(0)$ metric spaces satisfying the property of extension of local geodesics (see Section 4.1), which is not a circle nor a point and such that its universal cover $(X, d)$ is $\delta$-hyperbolic and satisfies $\operatorname{Ent}(X, d) \leq H$. We also denote by $\mathcal{M}(\delta, H, D)$ the subset of $\mathcal{M}(\delta, H)$ of metric spaces $(Y, d)$ such that $\operatorname{diam}(Y, d) \leq D$ and $\mathcal{M}(\delta, H, D) /$ $\sim$ the set of isometry classes of metric spaces in $\mathcal{M}(\delta, H, D)$.

Theorem 9.1. The set $\mathcal{M}(\delta, H) / \sim$ is complete for the Gromov-Hausdorff topology and so is $\mathcal{M}(\delta, H, D) / \sim$.

Proof. We just sketch the proof. The details can be found in [BCGS20a], Theorem 4.5. We consider a Cauchy sequence $\left(Y_{n}, d_{n}\right)$ in $\mathcal{M}(\delta, H) / \sim$. Let $\Gamma_{n}$ denote the fundamental group of $Y_{n}$. The following Lemma, due to G. Reviron is the main tool in the proof.

Lemma 9.2 ([Rev08]). Let $\left(Y_{n}, d_{n}\right)$ be a Cauchy sequence of compact metric spaces with universal cover $\left(X_{n}, d_{n}\right)$ and fundamental group $\Gamma_{n}$. Assume that the global systole of $\Gamma_{n}$ is uniformly bounded below, $\operatorname{sys}_{\Gamma_{n}}\left(X_{n}\right) \geq s_{0}>0$. Then, $\left(Y_{n}, d_{n}\right)$ converges for the Gromov-Hausdorff topology to a compact metric space $(Y, d)$. The limit space $(Y, d)$ has a universal cover $(X, d)$ satisfying $\operatorname{Ent}(X, d)=\lim \operatorname{Ent}\left(X_{n}, d_{n}\right)$. Moreover, the fundamental groups of $Y$ and $Y_{n}$ are isomorphic for $n$ large enough.

Indeed, we can verify that the Cauchy sequence $\left(Y_{n}, d_{n}\right)$ satisfies the hypothesis of Lemma 9.2, therefore $\left(Y_{n}, d_{n}\right)$ converges to a metric space $(Y, d)$ and it remains to verify that $(Y, d)$ is in $\mathcal{M}(\delta, H)$. In order to verify the hypotheses of Lemma 9.2 , we first observe that since $\left(Y_{n}, d_{n}\right)$ is locally $\operatorname{CAT}(0)$ and satisfies the property of extension of geodesics, then $\left(Y_{n}, d_{n}\right)$ has a universal cover $(X, d)$ which is CAT $(0)$, thus Busemann, cf. [BCGS20b], Lemma 6.41. On the other hand, since the sequence $\left(Y_{n}, d_{n}\right)$ is a Cauchy sequence and the diameter is 1-Lipschitz with respect to the Gromov-Hausdorff distance, there exists $D$ such that $\operatorname{diam}\left(Y_{n}, d_{n}\right) \leq D$. Hence, $\left(Y_{n}, d_{n}\right) \in \mathcal{M}(\delta, H, D)$ and by Lecture 8, Proposition 6.5, there exists $s_{0}:=s_{0}(\delta, H, D)>0$ such that $\operatorname{sys}_{\Gamma_{n}}\left(X_{n}\right) \geq s_{0}$. Lemma 9.2 then applies and $\left(Y_{n}, d_{n}\right)$ converges to a compact metric space $(Y, d)$. In order to end the proof of Theorem 9.1, we need to show that the limit space $(Y, d)$ is in $\mathcal{M}(\delta, H)$, i.e. is locally $\mathrm{CAT}(0)$, verifies the property of extension of local geodesics, and has a universal cover which is $\delta$-hyperbolic.This essentially follows from the continuity of the distance under Gromov Hausdorff convergence and from the fact that all these properties are defined by inequalities on expression involving the distance, we refer to [BCGS20a], Theorem 4.5 for details. We finally observe that $\mathcal{M}(\delta, H, D) / \sim$ is a closed subset of $\mathcal{M}(\delta, H)$ hence complete.

We now state a compactness Theorem.

Theorem 9.3. The set $\mathcal{M}(\delta, H, D) / \sim$ is compact for the Gromov-Hausdorff topology.

Proof. It suffices to show that $\mathcal{M}(\delta, H, D) / \sim$ is precompact since it is already complete. This will follow from the precompactness criterium that we describe now.

For $(Y, d)$ a compact metric space, and $\epsilon>0$, let us denote by $N_{\epsilon}(Y, d)$ be the minimal cardinality of an $\epsilon$-net of $(Y, d)$. A subset $\mathcal{M}^{\prime} \subset \mathcal{M}$ is precompact for the GromovHausdorff distance if and only if for every $\epsilon$, there exists $N_{\epsilon}$ such that for every $(Y, d) \in$ $\mathcal{M}^{\prime}$, then $\mathbf{N}_{\epsilon}(Y, d) \leq N_{\epsilon}$. 
We now apply this criterium to $\mathcal{M}^{\prime}=\mathcal{M}(\delta, H, D)$. Let us consider $(Y, d) \in \mathcal{M}(\delta, H, D)$ and $\pi:(X, d) \rightarrow(Y, d)$ the projection from the universal cover $(X, d)$ of $(Y, d)$. For every $\epsilon>0$, we have $N_{\epsilon}(Y, d) \leq N_{\epsilon}(B(x, D))$, where $y=\pi(x)$ is any point in $Y$ and $B(x, D) \subset X$ is the ball of radius $D$ centered at $x$ in $X$. We recall, from Lecture 4 that, for every $\lambda>1$,

$$
\begin{aligned}
N_{\epsilon}(B(x, D)) & =\operatorname{Cover}(x, \epsilon, D) \\
& \leq \operatorname{Pack}\left(x, \frac{\epsilon}{2}, D+\frac{\epsilon}{2}\right) \\
& \leq \operatorname{Pack}\left(x, \lambda \frac{\epsilon}{2}, \lambda\left(D+\frac{\epsilon}{2}\right)\right) .
\end{aligned}
$$

We deduce that for every $\lambda$ such that $\frac{\lambda \epsilon}{2}>D$,

$$
\begin{aligned}
N_{\epsilon}(B(x, D)) & \leq \operatorname{Pack}_{\Gamma}\left(x, \lambda \frac{\epsilon}{2}-D, \lambda\left(D+\frac{\epsilon}{2}\right)\right) \\
& \leq \frac{\mu_{x}^{\Gamma}\left(B\left(x, \lambda\left(D+\frac{\epsilon}{2}\right)\right)\right)}{\left.\mu_{x}^{\Gamma}\left(B\left(x, \lambda \frac{\epsilon}{2}-D\right)\right)\right)} \\
& \leq \operatorname{Pack}\left(x, \lambda \frac{\epsilon}{2}, \lambda\left(D+\frac{\epsilon}{2}\right)\right) .
\end{aligned}
$$

Choosing $\lambda=\frac{4 D}{\epsilon}$, we get

$$
\begin{aligned}
N_{\epsilon}(B(x, D)) & \leq \frac{\mu_{x}^{\Gamma}\left(B\left(x, \frac{4 D}{\epsilon}\left(D+\frac{\epsilon}{2}\right)\right)\right)}{\left.\mu_{x}^{\Gamma}(B(x, D))\right)} \\
& \leq C(\delta, H, D, \epsilon),
\end{aligned}
$$

where the last inequality comes from either Theorem 4.3 or 4.10 according to whether $\frac{4 D}{\epsilon}\left(D+\frac{\epsilon}{2}\right)<12(D+2 \delta)$ or $\frac{4 D}{\epsilon}\left(D+\frac{\epsilon}{2}\right) \geq 12(D+2 \delta)$ with $C(\delta, H, D, \epsilon)$ the corresponding constant. We therefore deduce that for every $(Y, d) \in \mathcal{M}(\delta, H, D)$ and $\epsilon>0$,

$$
N_{\epsilon}(Y, d) \leq N_{\epsilon}:=C(\delta, H, D, \epsilon),
$$

which concludes the prove of Theorem 9.3.

\subsection{Topological finiteness}

When we restrict the set of metric spaces under consideration to be also topological manifolds, we show that this set contains only finitely many topological types of manifolds. The next definitions are due to S. Ferry and play an important role in finiteness Theorems, [Fer94].

Definition 9.4. A continuous function $\rho:\left[0, R\left[\rightarrow \mathbf{R}_{+}\right.\right.$such that $\rho(0)=0$ and $\rho(t) \geq t$ is a contractibility function for a metric space $(Y, d)$ if for every $y \in Y$ and every $t, R$ with $0<t \leq R$, the ball $B(y, t)$ is contractible in $B(y, \rho(t))$. 
We also define

Definition 9.5. Let $\rho:\left[0, R\left[\rightarrow \mathbf{R}_{+}\right.\right.$be a continuous function such that $\rho(0)=0$. Let us define $\mathcal{M}^{\text {man }}(n, \rho)$ as the set of isometry classes of metric spaces $(Y, d)$ such that $Y$ is a topological manifold of dimension $n$ with $\rho$ as a contractibility function.

Theorem 9.6 ([Fer94], Theorem 1). Let $\mathcal{M}^{\prime} \subset \mathcal{M}^{\text {man }}(n, \rho)$ be a subset such that $\overline{\mathcal{M}^{\prime}}$ is compact in $\mathcal{M} / \sim$, then $\mathcal{M}^{\prime}$ contains finitely many topological types of manifolds.

Let us define $\mathcal{M}^{\text {man }}(n, \delta, H, D)$ the set of isometry classes of metric spaces in $\mathcal{M}(\delta, H, D)$ such that $Y$ is a topological manifold of dimension $n$. Theorem 9.6 has the following Corollary.

Corollary 9.7. The set $\mathcal{M}^{\text {man }}(n, \delta, H, D)$ contains finitely many topological types of manifolds.

Proof. Let $\pi:(X, d) \rightarrow(Y, d)$ be the universal cover of $(Y, d) \in \mathcal{M}^{\text {man }}(n, \delta, H, D)$ and $\Gamma$ the fundamental group of $Y$. We have shown that the global systole of $\Gamma$ is bounded below

$$
\operatorname{sys}_{\Gamma}(X) \geq s_{0}(\delta, H, D)>0 .
$$

Therefore, since $X$ is a $\mathrm{CAT}(0)$-space, for every $x \in X, B(x, t) \subset X$ is contractible. Moreover, for all $0<t \leq \frac{s_{0}}{2}$ the ball $B(x, t)$ is isometric to $B(y, t) \in Y$ where $y=\pi(x)$, therefore $\rho(t):\left[0, \frac{s_{0}}{2}\left[\rightarrow \mathbf{R}_{+}\right.\right.$, defined by $\rho(t)=t$, can be chosen as a contractibility function of $(Y, d)$.

\subsection{Growth and entropy of groups}

Let $(\Gamma, S)$ be a finitely generated group and $\left(\mathcal{G}(\Gamma, S), d_{S}\right)$ its Cayley graph. Notice that left multiplication induces an isometric action of $\Gamma$ on $\left(\mathcal{G}(\Gamma, S), d_{S}\right)$. We define the entropy of $\Gamma$ with respect to $S$ by

Definition 9.8. $\operatorname{Ent}(\Gamma, S)=\lim _{R \rightarrow \infty} \frac{1}{R} \log \#\left\{\gamma \in \Gamma \mid d_{S}(e, \gamma) \leq R\right\}$.

Notice that for a finitely generated group $\Gamma$, if $\operatorname{Ent}(\Gamma, S)>0$ for one generating set $S$, then $\operatorname{Ent}(\Gamma, S)>0$ for every $S$, and that $\operatorname{Ent}(\Gamma, S)>0$ if and only if $\Gamma$ has exponential growth. On the other hand, if $\Gamma$ has polynomial growth, then $\operatorname{Ent}(\Gamma, S)=0$ for every generating set $S$.

Examples 9.9. 1. When $\Gamma$ is the non abelian free group on $k$ generators $\mathbf{F}_{k}$ and $\mathbf{S}_{k}$ the standard generating set, $\operatorname{Ent}\left(\mathbf{F}_{k}, \mathbf{S}_{k}\right)=\log (2 k-1)$.

2. When $\Gamma=\mathbf{Z}^{k}$, we have $\operatorname{Ent}\left(\mathbf{Z}^{k}, S\right)=0$ for every generating set $S$ since $\mathbf{Z}^{k}$ has polynomial growth.

3. Let us consider the matrix $A=\left(\begin{array}{ll}2 & 1 \\ 1 & 1\end{array}\right) \in S L(2, \mathbf{Z})$ and define the group $\Gamma_{A}=\mathbf{Z} \ltimes \mathbf{Z}^{2}$ where the product law is given by $(n,(p, q)) \cdot\left(n^{\prime},\left(p^{\prime}, q^{\prime}\right)\right)=\left(n+n^{\prime},(p, q)+\left(p^{\prime}, q^{\prime}\right) A^{n}\right)$. It can easily be shown that $\Gamma_{A}$ is a solvable non nilpotent group of exponential growth. We leave it as an exercise. 
However, the entropy depends on the finite generating set $S$ and is in general impossible to compute. Taking the infimum over all finite generating sets of a group gives the "algebraic entropy" Ent $\Gamma$ of $\Gamma$ defined as follows.

Definition 9.10. Ent $\Gamma:=\inf _{S} \operatorname{Ent}(\Gamma, S)$, where $S$ is a finite generating sets of $\Gamma$.

Answering a question of M. Gromov, Wilson constructed an example of a finitely generated group $\Gamma$ of exponential i.e. $\operatorname{Ent}(\Gamma, S)>0$ for every finite generating set $S$, but with vanishing algebraic entropy, Ent $\Gamma=0$. On the other hand, D. V. Osin has shown that non nilpotent solvable groups have positive algebraic entropy, cf. [Osi03]. M. Koubi also proved that when $\Gamma$ is a hyperbolic group, then $\operatorname{Ent} \Gamma>0$, cf. [Kou98].

The following observation is fundamental for getting lower bounds on the entropy.

Lemma 9.11. Let $(\Gamma, S)$ be a finitely generated group. Assume that there exist two elements $a, b \in \Gamma$ generating a non abelian free subgroup of $\Gamma$. Then,

$$
\operatorname{Ent}(\Gamma, S) \geq \frac{\log 3}{\sup \left(l_{S}(a), l_{S}(b)\right)},
$$

where $l_{S}(a)=d_{S}(e, a)$ and $l_{S}(b)=d_{S}(e, b)$. Similarily, if the elements $a, b \in \Gamma$ generate a non abelian free semi-group, we have

$$
\operatorname{Ent}(\Gamma, S) \geq \frac{\log 2}{\sup \left(l_{S}(a), l_{S}(b)\right)}
$$

Proof. We prove the Lemma in the case where the elements $a, b$ generate a non abelian free group; the second case is identical. We let $L_{S}(a, b):=\sup \left(l_{S}(a), l_{S}(b)\right)$. We also write $\mathbf{F}_{a, b}$ for the non abelian free group on $a$ and $b$ and $S_{a, b}$ the canonical generating set of $\mathbf{F}_{a, b}$, namely, $S_{a, b}:=\left\{a^{ \pm 1}, b^{ \pm 1}\right\}$. For $k \in \mathbf{N}$, we let $\mathbf{B}_{S_{a, b}}(k):=\left\{\gamma \in \mathbf{F}_{a, b} \mid l_{S_{a, b}}(\gamma) \leq k\right\}$ and $B_{S}(k):=\left\{\gamma \in \Gamma \mid l_{S}(\gamma) \leq k\right\}$, we have

$$
B_{S_{a, b}}(k) \subset B_{S}\left(L_{S}(a, b) k\right),
$$

therefore,

$$
\log 3=\lim _{k \rightarrow \infty} \frac{1}{k} \log \left(\# B_{S_{a, b}}(k)\right) \leq \lim _{k \rightarrow \infty} \frac{1}{k} \log \left(\# B_{S}\left(L_{S}(a, b) k\right)\right),
$$

hence

$$
\log 3 \leq L_{S}(a, b) \operatorname{Ent}(\Gamma, S)
$$

This Lemma shows that getting a lower bound on the algebraic entropy of a group $\Gamma$ amounts to finding, for every finite generating set $S$ of $\Gamma$, two elements $a, b \in \Gamma$ generating a non abelian free group and with $L_{S}(a, b)$ not too large. For linear groups over a field $\mathbf{K}$ that is, $\Gamma \subset G L(n, \mathbf{K})$, the Tits alternative proves the existence of free subgroup as soon as $\Gamma$ is not virtually solvable. 
Theorem 9.12 ([Tit72]). Let $\Gamma$ be a finitely generated group in $G L(n, \mathbf{K})$. Then, either $\Gamma$ is virtually solvable or $\Gamma$ contains a non abelian free subgroup.

In particular, non virtually solvable linear groups have exponential growth. In the following statement, it is shown that for non virtually solvable linear groups, one can find pairs of elements generating non abelian free semi-groups at uniform bounded distance from the Identity with respect to all generating sets.

Theorem 9.13 ([EMO05]). Let $\Gamma$ be a finitely generated linear group $G L(n, \mathbf{K})$ over a field of characteristic 0 . If $\Gamma$ is not virtually solvable, there exists $N \in \mathbf{N}^{*}$ such that for every generating set $S$, there exists $a, b \in B_{S}(N)$ such that $a, b$ generates a non abelian free semi-group. In particular, the algebraic entropy of $\Gamma$ is positive, Ent $\Gamma>0$.

For solvable groups, D. V. Osin proved the following alternative,

Theorem 9.14 ([Osi03]). Let $\Gamma$ be a finitely generated (non necessarily linear) group. Then either $\Gamma$ is virtually nilpotent or $\Gamma$ has positive algebraic entropy.

From this discussion, we get the following corollary for linear groups $\Gamma \in G L(n, \mathbf{K})$ over a field of characteristic 0: either $\Gamma$ is virtually nilpotent or $\operatorname{Ent} \Gamma>0$.

A natural question is whether, given a class $\mathcal{C}$ of groups with exponential growth, there exists a universal positive constant $C>0$ such that

$$
\text { Ent } \Gamma \geq C
$$

for every group $\Gamma \in \mathcal{C}$.

\section{Lecture 10}

In this lecture we consider the class $\mathcal{C}$ of groups $\Gamma$ which have an action on some $\delta$ hyperbolic metric space $(X, d)$ with $\operatorname{Ent}(X, d) \leq H$ and such that diam $X / \Gamma \leq D$. We show that there exists a constant $C(\delta, H, D)>0$ such that the algebraic entropy of every $\Gamma \in \mathcal{C}$ satisfies $\operatorname{Ent} \Gamma \geq C(\delta, H, D)$.

\subsection{Algebraic entropy of groups acting on hyperbolic metric spaces}

For $\Gamma$ a finitely generated group, $S$ a subset of $\Gamma$ and $N \in \mathbf{N}$, we denote by $S^{N}$ the set of elements $\gamma \in \Gamma$ that can be written as a product of at most $N$ elements in $S$. Given two elements $a, b$ in $\Gamma$, we write $\langle a, b\rangle_{+}$the semi-group generated by $a$ and $b$.

Theorem 10.1. There exists $N_{0}:=N_{0}(\delta, H, D)>0$ such that for every group $\Gamma$ acting properly discontinuously on a $\delta$-hyperbolic non elementary metric space $(X, d)$ with $\operatorname{Ent}(X, d) \leq H$ and such that $\operatorname{diam}(X, d) \leq D$, we have:

1. For every subset $S$ of $\Gamma$ such that the group $\langle S\rangle$ generated by $S$ is not virtually cyclic, there exists $\gamma \in S^{3 N_{0}}$ and $\sigma \in S$ such that one of the semi-groups $\left\langle\gamma^{14}, \sigma \gamma^{14} \sigma^{-1}\right\rangle_{+}$or $\left\langle\gamma^{14}, \sigma \gamma^{-14} \sigma^{-1}\right\rangle_{+}$is free. 
2. The algebraic entropy of any finitely generated non virtually cyclic subgroup $\Gamma^{\prime} \leq \Gamma$

$$
\operatorname{Ent} \Gamma^{\prime} \geq \frac{\log 2}{42 N_{0}+2}
$$

In particular Ent $\Gamma \geq \frac{\log 2}{42 N_{0}+2}$.

Proof. We sketch the proof; the details can be found in [BCGS20a], Proposition 5.18. The proof relies on the following theorem by Breuillard-Green-Tao that we recall for convenience.

Theorem 10.2 ([BGT12]). For every $p \in \mathbf{N} \backslash\{0\}$, there exists $N(p) \in \mathbf{N} \backslash\{0\}$ such that the following holds: for every group $G$ and every generating set $S$ of $G$, if there exists a finite subset $A \subset G$ such that

1. $S^{N(p)} \subset A$

2. $\frac{\# A \cdot A}{\# A} \leq p$,

then $G$ is virtually nilpotent.

We apply Theorem 10.2 to $G=\langle S\rangle \leq \Gamma$. Let $R_{0}:=20(D+2 \delta)$ and $x \in X$. We define

$$
A:=\left\{g \in G \mid d(x, g x) \leq R_{0}\right\} .
$$

By the triangle inequality, we have

$$
A \cdot A \subset\left\{g \in G \mid d(x, g x) \geq 2 R_{0}\right\},
$$

We then have

$$
\begin{aligned}
\frac{\# A \cdot A}{\# A} & \leq \frac{\mu_{x}^{G}\left(B\left(x, 2 R_{0}\right)\right)}{\mu_{x}^{G}\left(B\left(x, R_{0}\right)\right)} \\
& \leq \frac{\mu_{x}^{\Gamma}\left(B\left(x, 2 R_{0}+\frac{R_{0}}{2}\right)\right)}{\mu_{x}^{\Gamma}\left(B\left(x, \frac{R_{0}}{2}\right)\right)} \\
& \leq p:=p(\delta, H, D)=3 \cdot 5^{\frac{25}{4}} e^{300 H(D+2 \delta)},
\end{aligned}
$$

where the second inequality comes from the Bishop-Gromov Theorem for subgroups, Corollary 4.9 and the third one from the Bishop-Gromov Theorem 1.8 with $(r, R)$ replaced by $\left(\frac{R_{0}}{2}, \frac{5}{2} R_{0}\right)$. Since the group $G$ is not virtually cyclic, we have by Theorem 10.2

$$
S^{N(p))} \not \subset A .
$$

Therefore, setting $N_{0}:=N_{0}(\delta, H, D)=N(p(\delta, H, D))$, there exists $\gamma_{0} \notin A$ such that

$$
\gamma_{0} \in S^{N_{0}} \text { and } \quad d\left(x, \gamma_{0} x\right)>R_{0} \geq \frac{31 \delta}{2} .
$$

Now, given a finite subset $\Sigma$ of $G$, we define 
Definition 10.3. $L(\Sigma):=\inf _{x \in X} \max _{\gamma \in \Sigma} d(x, \gamma x)$, and $L(\gamma):=L(\{\gamma\})$.

For a non trivial isometry $\gamma$ of $(X, d)$, we define its "asymptotic displacement" $\ell(\gamma)$ by

Definition 10.4. $\ell(\gamma):=\lim _{k \rightarrow \infty} \frac{d\left(x, \gamma^{k} x\right)}{k}$.

The reader can easily check, using the triangle inequality, that the limit exists and does not depend on $x \in X$. Notice that

$$
\ell(\gamma) \leq L(\gamma)
$$

and that for $k \in \mathbf{Z}^{*}$,

$$
\ell\left(\gamma^{k}\right)=|k| \ell(\gamma)
$$

By (13), we have

$$
L\left(S^{N_{0}}\right) \geq \frac{31 \delta}{2} .
$$

The following Lemma says that if a subset $\Sigma$ of $G$ has a large joint displacement, then there exists an element $\gamma \in \Sigma^{3}$ with large asymptotic displacement.

Lemma 10.5. If $L\left(S^{N_{0}}\right) \geq \frac{31 \delta}{2}$, then there exits $\gamma_{1} \in S^{3 N_{0}}$ such that $\ell\left(\gamma_{1}\right) \geq \delta$.

Proof. We skip the proof of this Lemma and refer to [BCGS20b], Theorem 4.17.

Now, since $G$ is not virtually cyclic, there exists $\sigma \in S$ such that the group $\left\langle\gamma_{1}, \sigma \gamma_{1} \sigma^{-1}\right\rangle$ is not virtually cyclic.

Claim 10.6. One of the semi-groups $\left\langle\gamma_{1}^{14}, \sigma \gamma_{1}^{14} \sigma^{-1}\right\rangle_{+}$or $\left\langle\gamma_{1}^{14}, \sigma \gamma_{1}^{-14} \sigma^{-1}\right\rangle_{+}$is free.

The Claim corresponds to the first part of Theorem 10.1. The second part is a consequence of Lemma 9.11. Let us assume for example that the semi-group $\left\langle\gamma_{1}^{14}, \sigma \gamma_{1}^{14} \sigma^{-1}\right\rangle_{+}$ is free and write $\alpha:=\gamma_{1}^{14}, \beta:=\sigma \gamma_{1}^{14} \sigma^{-1}$. Since $\gamma_{1} \in S^{3 N_{0}}$, we have $l_{S}(\alpha) \leq 14\left(3 N_{0}\right)$ and $l_{S}(\beta) \leq 14\left(3 N_{0}\right)+2$. By the second part of Lemma 9.11, we get

$$
\operatorname{Ent}(\Gamma, S) \geq \frac{\log 2}{42 N_{0}+2} .
$$

It remains to prove Claim 10.6. This Claim will be basically a consequence of the following "ping-pong Lemma" that we now formulate in our particular setting.

Lemma 10.7. Let $\alpha, \beta$ be two hyperbolic isometries of $(X, d)$. We assume that there exist subsets $U_{\alpha}^{ \pm}, U_{\beta}^{ \pm}$of $X$ and $x \in X \backslash\left(U_{\alpha}^{+} \cup U_{\beta}^{+}\right)$such that

$$
\begin{aligned}
& U_{\alpha}^{+} \subset X \backslash\left(U_{\beta}^{-} \cup U_{\beta}^{+}\right), \\
& U_{\beta}^{+} \subset X \backslash\left(U_{\alpha}^{-} \cup U_{\alpha}^{+}\right),
\end{aligned}
$$

and such that $\alpha^{k}\left(\{x\} \cup U_{\beta}^{+}\right) \subset U_{\alpha}^{+}$and $\beta^{k}\left(\{x\} \cup U_{\alpha}^{+}\right) \subset U_{\beta}^{+}$for every $k \in \mathbf{N}^{*}$, then the semi-group $\langle\alpha, \beta\rangle_{+}$generated by $\alpha$ and $\beta$ is free. 
Proof. We want to prove that, given a non trivial word $w(\alpha, \beta)$ in $\alpha$ and $\beta$, then $w(\alpha, \beta) \neq I d$. Let us consider a non trivial word $w(\alpha, \beta)$. We can assume without loss of generality that the word $w(\alpha, \beta)$ starts with $\alpha$. There are two cases.

1. $w(\alpha, \beta)=\alpha^{n_{1}} \beta^{p_{1}} \cdots \alpha^{n_{k}} \beta^{p_{k}}$;

2. $w(\alpha, \beta)=\alpha^{n_{1}} \beta^{p_{1}} \cdots \alpha^{n_{k}}$

where $n_{i}, p_{i} \in \mathbf{N}^{*}$.

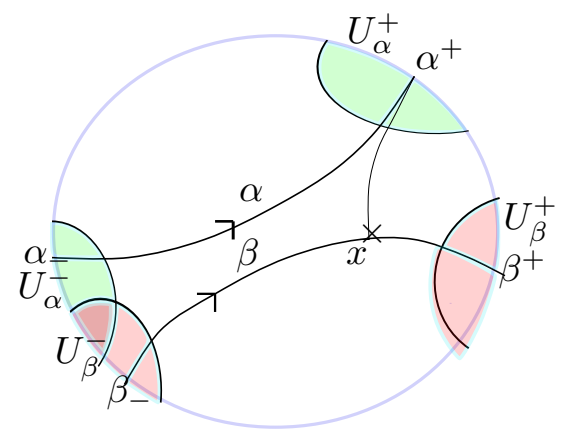

Figure 10.1: ping-pong

It is straightforward to check from the assumptions that $w(\alpha, \beta) \cdot x \in U_{\alpha}^{+}$. Therefore, since $x \notin U_{\alpha}^{+}$, we deduce that $w(\alpha, \beta) \neq I d$.

We now briefly sketch how to deduce the claim from the ping-pong lemma. The details can be found in [BCGS20b], Proposition 4.6 and 4.9. Writing $\alpha:=\gamma_{1}^{14}$ and $\beta:=\sigma \gamma_{1}^{14} \sigma^{-1}$, we observe that $\ell(\alpha) \geq 14 \delta$ and $\ell(\beta) \geq 14 \delta$ by Lemma 10.5 and (14). Now, the idea is the following: since the asymptotic displacement of $\alpha$ and $\beta$ is large with respect to $\delta$ and since the fixed point sets $\left\{\alpha^{ \pm}\right\}$, and $\left\{\beta^{ \pm}\right\}$of $\alpha, \beta$ are disjoint, one can construct neighbourhoods of their fixed points such as in the ping-pong Lemma for $\{\alpha, \beta\}$ or $\left\{\alpha, \beta^{-1}\right\}$. Given $\gamma$ an isometry of $(X, d)$ and $x \in X$, let us define the Dirichlet domain of $\gamma$ at $x$ as

$$
D_{\gamma}(x):=\left\{y \in X \mid \min _{k \in \mathbf{Z}} d\left(y, \gamma^{k} x\right)=d(y, x)\right\},
$$

and

$$
U_{\gamma}^{ \pm}(x):=\cup_{k \in \mathbf{N}^{*}} D_{\gamma^{ \pm k}}(x) .
$$

We choose an axis $c_{\beta}$ of $\beta$. We can show that, up to a change of orientation of $\alpha$, there is a point $x$ on $c_{\beta}$ which is roughly a projection of $\alpha^{+}$onto $c_{\beta}$ such that for every $p, q \in\left(\mathbf{Z}^{*} \times \mathbf{Z}^{*}\right) \backslash\left(\mathbf{Z}^{-} \times \mathbf{Z}^{-}\right)$,

$$
d\left(\alpha^{p} x, \beta^{q} x\right)>\max \left\{d\left(x, \alpha^{p} x\right), d\left(x, \beta^{q} x\right)\right\}+2 \delta .
$$

We skip the proof of (17) and refer to [BCGS20b], Proposition 4.9, for the details. Now, we set $U_{\alpha}^{ \pm}:=U_{\alpha}^{ \pm}(x)$ and $U_{\beta}^{ \pm}:=U_{\beta}^{ \pm}(x)$ and prove that the hypotheses of the Ping-Pong 
lemma 10.1 are satisfied. By definition we have $x \notin U_{\alpha}^{+} \cup U_{\beta}^{+}$and $\alpha^{k} x \in U_{\alpha}^{+}, \beta^{k} x \in U_{\beta}^{+}$ for every $k \in \mathbf{N}^{*}$. Let $y \in U_{\beta}^{+} \cup U_{\beta}^{-}$. We first observe that, by definition, there exists $k \in \mathbf{Z}^{*}$ such that $y \in D_{\beta}\left(\beta^{k} x\right)$ hence

$$
d\left(y, \beta^{k} x\right) \leq d(y, x) .
$$

Let us recall the quadrangle property of $\delta$-hyperbolic metric spaces (see Section 2.1) for the points $y, \alpha^{p} x, x$ and $\beta^{k} x$ with $k, p \in \mathbf{Z}^{*}$,

$$
d(y, x)+d\left(\alpha^{p} x, \beta^{k} x\right) \leq \max \left\{d\left(y, \beta^{k} x\right)+d\left(x, \alpha^{p} x\right) ; d\left(y, \alpha^{p} x\right)+d\left(x, \beta^{k} x\right)\right\}+2 \delta .
$$

By (17) and (19), we deduce

$$
d(y, x)<\max \left\{d\left(y, \beta^{k} x\right) ; d\left(y, \alpha^{p} x\right)\right\},
$$

and since $d(y, x) \geq d\left(y, \beta^{k} x\right)$, we get that $d(y, x)<d\left(x, \alpha^{p} x\right)$, for every $p \in \mathbf{Z}^{*}$, which means that

$$
y \in D_{\alpha}(x)=X \backslash\left(U_{\alpha}^{+} \cup U_{\alpha}^{-}\right) .
$$

Thus, $U_{\beta}^{+} \cup U_{\beta}^{-} \subset X \backslash\left(U_{\alpha}^{+} \cup U_{\alpha}^{-}\right)$. We similarly verify the same hypothesis with the roles of $\alpha$ and $\beta$ reversed. This proves that the hypotheses of the Ping-Pong lemma are satisfied and concludes the proof of Theorem 10.1.

\subsection{Entropy of a $\delta$-hyperbolic space with a group action}

So far, we have investigated properties of groups $\Gamma$ acting by isometries on a $\delta$-hyperbolic metric space $(X, d)$ such that $\operatorname{Ent}(X, d) \leq H$ and $\operatorname{diam}(X / \Gamma)$. In the other way round, we have the

Theorem 10.8. Let $(X, d)$ be a non elementary $\delta$-hyperbolic metric space and $\Gamma$ a group acting properly by isometries on $(X, d)$ such that $\operatorname{diam} X / \Gamma \leq D$. Then

$$
\operatorname{Ent}(X, d) \geq \frac{\log 2}{27 \delta+10 D}
$$

We skip the proof of this Theorem and refer to [BCGS20b], Proposition 5.10.

Coming back to the groups, let us consider a finitely generated group $(\Gamma, S)$ which is $\delta$-hyperbolic with respect to the distance $d_{S}$. As a direct Corollary of Theorem 10.8, we get

$$
\operatorname{Ent}(\Gamma, S) \geq \frac{\log 2}{27 \delta+10} .
$$

Example 10.9. Let us consider the regular tree of valence 4 which we see as the Cayley graph $X:=\mathcal{G}(\mathbf{F}(\alpha, \beta), \mathbf{S}(\alpha, \beta))$ of the non abelian free group on two generators $\Gamma:=$ $\mathbf{F}(\alpha, \beta)$ with respect to the generating set $\left\{\alpha^{ \pm 1}, \beta^{ \pm 1}\right\}$. Given $a>0$ and $b>0$, we define the distance $d_{a, b}$ on $X$ by setting the length of each edge $\left(\gamma, \alpha^{ \pm 1} \gamma\right)$ equal to a and each edge $\left(\gamma, \beta^{ \pm 1} \gamma\right)$ equal to $b$. For every $a>0, b>0$, the metric space $\left(X, d_{a, b}\right)$ is 
$\delta$-hyperbolic with $\delta=0$ and the group $\Gamma=\mathbf{F}(\alpha, \beta)$ acts isometrically on $\left(X, d_{a, b}\right)$. Notice that

$$
\operatorname{diam}\left(\left(X, d_{a, b}\right) / \Gamma\right)=\frac{a+b}{2} .
$$
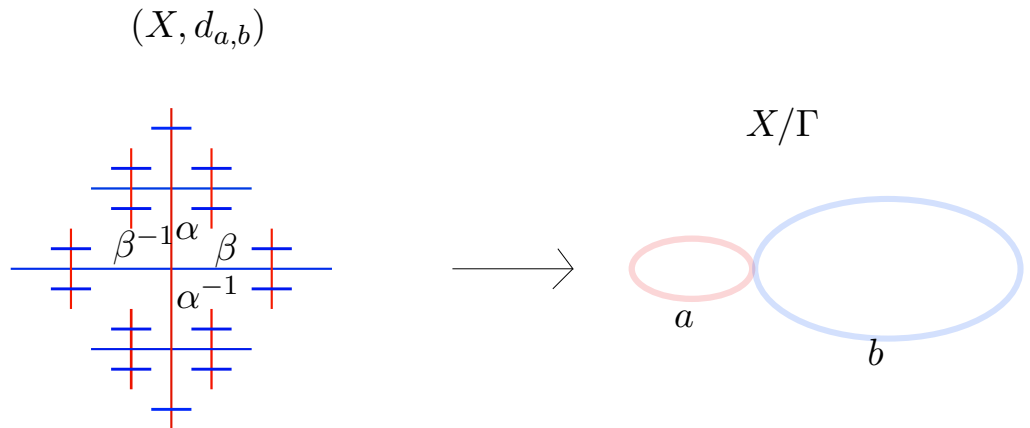

Figure 10.2: Example

Theorem 10.8 implies

$$
\operatorname{Ent}\left(X, d_{a, b}\right) \geq \frac{\log 2}{5(a+b)} .
$$

We leave to the reader to verify that, when $b=1$ is fixed, then

$$
\lim _{a \rightarrow 0} \operatorname{Ent}\left(X, d_{a, b}\right)=+\infty,
$$

and

$$
\lim _{a \rightarrow \infty} \operatorname{Ent}\left(X, d_{a, b}\right)=0 .
$$

\section{References}

[ABN86] A. D. Aleksandrov, V. N. Berestovskij, and I. G. Nikolaev. Generalized Riemannian spaces. Russ. Math. Surv., 41(3):1-54, 1986.

[Bas72] H. Bass. The degree of polynomial growth of finitely generated nilpotent groups. Proc. London Math. Soc. (3), 25:603-614, 1972.

[BBI01] D. Burago, Yu. Burago, and S. Ivanov. A course in metric geometry, volume 33. Providence, RI: American Mathematical Society (AMS), 2001.

[BCGS20a] G. Besson, G. Courtois, S. Gallot, and A. Sambusetti. Compactness and Finiteness Theorems for Gromov-Hyperbolic spaces. Preprint, 2020.

[BCGS20b] G. Besson, G. Courtois, S. Gallot, and A. Sambusetti. Curvature-Free Margulis Lemma for Gromov-Hyperbolic Spaces. arXiv: 1712.08386v2, 2020. 
[BE85] D. Bakry and M. Emery. Diffusions hypercontractives. Sémin. de probabilités XIX, Univ. Strasbourg 1983/84, Proc., Lect. Notes Math. 1123, 177-206, 1985.

[BGS85] W. Ballmann, M. Gromov, and V. Schroeder. Manifolds of nonpositive curvature. Progress in Mathematics, 61. Boston-Basel-Stuttgart: Birkhäuser. iv, 263, 1985.

[BGT12] E. Breuillard, B. Green, and T. Tao. The structure of approximate groups. Publ. Math., Inst. Hautes Étud. Sci., 116:115-221, 2012.

[BH99] M. R. Bridson and A. Haefliger. Metric spaces of non-positive curvature. Berlin: Springer, 1999.

[BZ88] Yu. D. Burago and V. A. Zalgaller. Geometric inequalities. Transl. from the Russian by A. B. Sossinsky. Springer-Verlag, Berlin, 1988.

[CDP90] M. Coornaert, Th. Delzant, and A. Papadopoulos. Géométrie et théorie des groupes. Les groupes hyperboliques de Gromov. (Geometry and group theory. The hyperbolic groups of Gromov). Springer-Verlag, Berlin, 1990.

[Cou15] G. Courtois. Lemme de Margulis à courbure de Ricci minorée (d'après Vitali Kapovitch et Burkhard Wilking). In Séminaire Bourbaki. Volume 2013/2014. Exposés 1074-1088, pages 25-56. Société Mathématique de France (SMF), Paris, 2015.

[EMO05] A. Eskin, S. Mozes, and H. Oh. On uniform exponential growth for linear groups. Invent. Math., 160(1):1-30, 2005.

[Fer94] S. Ferry. Topological finiteness theorems for manifolds in Gromov-Hausdorff space. Duke Math. Journ., 74:95-106, 1994.

[Gal83a] S. Gallot. A Sobolev Inequality and some applications, Proceedings du Séminaire Franco-Japonais de Kyoto 1981. Kaigai Publications, Tokyo, pages 45-55, 1983.

[Gal83b] S. Gallot. Inégalités isopérimétriques, courbure de Ricci et invariants géométriques. II. C. R. Acad. Sci. Paris Sér. I Math., 296(8):365-368, 1983.

[GdlH90] E. Ghys and P. de la Harpe, editors. Sur les groupes hyperboliques d'après Mikhael Gromov. (On the hyperbolic groups à la M. Gromov). Boston, MA: Birkhäuser, 1990.

[Gro81] M. Gromov. Groups of polynomial growth and expanding maps (with an appendix by Jacques Tits). Publ. Math., Inst. Hautes Étud. Sci., 53:53-78, 1981.

[Gro87] M. Gromov. Hyperbolic groups. Essays in group theory, Publ., Math. Sci. Res. Inst. 8, 75-263 (1987)., 1987. 
[Gro07] M. Gromov. Metric structures for Riemannian and non-Riemannian spaces. Transl. from the French by S. M. Bates. With appendices by M. Katz, P. Pansu, and S. Semmes. Edited by J. Lafontaine and P. Pansu. Basel: Birkhäuser, 2007.

[Gui71] Y. Guivarc'h. Groupes de Lie à croissance polynomiale. C. R. Acad. Sci. Paris Sér. A-B, 272:A1695-A1696, 1971.

[Kou98] M. Koubi. Croissance uniforme dans les groupes hyperboliques. Ann. Inst. Fourier (Grenoble), 48(5):1441-1453, 1998.

[KW11] V. Kapovitch and B. Wilking. Structure of fundamental groups of manifolds with Ricci curvature bounded below. arxiv 1105.5955, 2011.

[Mar75] G. A. Margulis. Discrete groups of motions of manifolds of non-positive curvature. Proc. int. Congr. Math., Vancouver 1974, Vol. 2, 21-34, 1975.

[Osi03] D. V. Osin. The entropy of solvable groups. Ergodic Theory Dynam. Systems, 23(3):907-918, 2003.

[Rev08] G. Reviron. Topological rigidity under the hypothesis of bounded entropy and applications. Comment. Math. Helv., 83(4):815-846, 2008.

[Tit72] J. Tits. Free subgroups in linear groups. J. Algebra, 20:250-270, 1972.

[Vil09] C. Villani. Optimal transport. Old and new, volume 338. Berlin: Springer, 2009 . 


\section{Index}

CAT(0)-space, 44, 45, 47, 48, 50

$\delta$-hyperbolic, 6

$\delta$-thin, 6

Algebraic Entropy, 51, 52

Betti number, 17, 18

Bishop-Gromov, 9, 10, 21, 25

Busemann space, 21, 22, 24, 25, 33, 35, $42,44,45,47$

Cayley graph, 8

Collar, 33, 34

Covering, 23

Distance

Gromov-Hausdorff, 4

Entropy, 8

Algebraic, 51, 52

Extension of Geodesics, 22, 44, 48

Finitely Presented Group, 39

Finiteness Theorem, 42, 43, 47, 49

Gromov-Hausdorff topology, 4

Group

Finitely Presented, 39

Marked, 36, 39-42

Growth, 26, 27, 47, 50, 52

Homology group, 15-17

Inequality

Bishop-Gromov, 9, 10, 21, 25

Quadrangle, 10, 56

Lemma

Collar, 33, 34

Margulis, 26, 28

Ping-Pong, 54-56

Margulis Lemma, 26, 28

Marked group, 36, 39-42
Packing, 19, 23, 24, 47

Ping-Pong Lemma, 54-56

Quadrangle Inequality, 10, 56

Space

$\mathrm{CAT}(0), 44,45,47,48,50$

Busemann, 21, 22, 24, 25, 33, 35, 42, $44,45,47$

Systole, 26, 30-33, 48, 50

Thin-Thick Decomposition, 30, 33

Topological Finiteness, 49 